TRANSACTIONS OF THE

AMERICAN MATHEMATICAL SOCIETY

Volume 349, Number 8, August 1997, Pages 3105-3148

S 0002-9947(97)01860-6

\title{
MATRIX POLYNOMIALS AND THE INDEX PROBLEM FOR ELLIPTIC SYSTEMS
}

\author{
B. ROWLEY
}

\begin{abstract}
The main new results of this paper concern the formulation of algebraic conditions for the Fredholm property of elliptic systems of P.D.E.'s with boundary values, which are equivalent to the Lopatinskii condition. The Lopatinskii condition is reformulated in a new algebraic form (based on matrix polynomials) which is then used to study the existence of homotopies of elliptic boundary value problems. The paper also contains an exposition of the relevant parts of the theory of matrix polynomials and the theory of elliptic systems of P.D.E.'s.
\end{abstract}

\section{INTRODUCTION}

Let $\mathcal{A}$ denote an elliptic operator in $\Omega$ and let $\mathcal{B}$ be a boundary operator, where $\Omega$ is a bounded domain in $\mathbb{R}^{n}$. In this paper several versions of the algebraic condition for the Fredholm property of $(\mathcal{A}, \mathcal{B})$ are formulated, equivalent to the Lopatinskii condition of [Lo]. The main new result is the following. A square matrix function $\Delta_{\mathcal{B}}^{+}$defined on the unit cotangent bundle of $\partial \Omega$ is constructed from the principal symbols of the coefficients of the boundary operator and a spectral pair for the family of matrix polynomials associated with the principal symbol of the elliptic operator. The Lopatinskii condition is equivalent to the following condition: the function $\Delta_{\mathcal{B}}^{+}$must have invertible values.

The proofs use the theory of matrix polynomials due to Gohberg, Lancaster, Rodman, and others; for instance, see [GLR], Chapter 14 in [LT], and Chapter 6 in $[\mathrm{R}]$.

There is a natural map defined by $(\mathcal{A}, \mathcal{B}) \mapsto \mathcal{A}$, going from the space of elliptic boundary value problems to the space of elliptic operators. Now let $\mathcal{A}_{\tau}, 0 \leq \tau \leq 1$, be a homotopy of elliptic operators and let $\mathcal{B}_{0}$ be a boundary operator such that $\left(\mathcal{A}_{0}, \mathcal{B}_{0}\right)$ satisfies the Lopatinksii condition. We will show that the given homotopy of elliptic operators can be lifted to a homotopy $\left(\mathcal{A}_{\tau}, \mathcal{B}_{\tau}\right)$ in the space of elliptic boundary value problems satisfying the Lopatinskii condition. This result is proved in Theorem 7.3 and was motivated by the article $[\mathrm{Ge}]$. The key element in the proof, i.e. the construction of the boundary operators $\mathcal{B}_{\tau}$, requires that we use pseudodifferential operators on $\partial \Omega$ and the theory of matrix polynomials mentioned above.

Received by the editors August 16, 1994 and, in revised form, February 12, 1996.

1991 Mathematics Subject Classification. Primary 35J45, 35J55, 15A22.

Key words and phrases. Elliptic boundary value problems, matrix polynomials, Lopatinskii condition, Fredholm property.

The author wishes to acknowledge that the abstract, the introduction and parts of $\S \S 2$ and 3 were revised due to the helpful remarks and suggestions of the referee.

(C) 1997 American Mathematical Society 
The existence of homotopies is crucial for the index problem for elliptic systems, which is to find a formula for the index of the associated Fredholm operator. The results of the present paper (in particular Theorem 7.3) will be used by the author in a subsequent paper (see [Ro2]) to prove an index formula for elliptic systems in the plane, in terms of the winding number of the determinant of $\Delta_{\mathcal{B}}^{+}$.

In $\S 1$ we write out the definition of elliptic systems in the sense of DouglisNirenberg and also state the main properties of pseudo-differential operators which are needed here. Section 2 contains an exposition of the relevant parts of the theory of matrix polynomials. The results in this section are not mathematically new; however, the presentation of several constructions and proofs in this section is novel, and they are used later in an essential way. (See also [Ro1].) In $\S 3$ we formulate the algebraic condition for the Fredholm property in various equivalent forms (see Theorem 3.3). In $\S \S 4$ and 5 some technical results are developed concerning families of matrix polynomials, which are used to prove Theorem 7.3 and also two theorems in $\S 6$ of Agranovič and Dynin type, the first one on comparing the index of two boundary value problems having the same elliptic operator and the second on reducing the transversal order of a boundary operator.

A remark on the way in which equations are cross-referenced between sections: the notation (5-8) means equation (8) in $\S 5$.

\section{Elliptic systems of Douglis-Nirenberg type}

Let $\Omega$ be a bounded domain in $\mathbb{R}^{n}$, a domain being a connected, open set. We will consider systems of linear differential equations $\mathcal{A}(x, D) u(x)=f(x), x \in \Omega$, where $u$ and $f$ are $p$-vector functions and

$$
\mathcal{A}(x, D)=\left[A_{i j}(x, D)\right]_{p \times p}, \quad x \in \bar{\Omega},
$$

is a $p \times p$ matrix, such that the elements, $A_{i j}$, are linear differential operators

$$
A_{i j}(x, D)=\sum_{|\alpha| \leq \alpha_{i j}} a_{i j}^{(\alpha)}(x) D^{\alpha}
$$

with smooth coefficients, $a_{i j}^{(\alpha)} \in C^{\infty}(\bar{\Omega})$. Here the usual multi-index notation is being used: $D^{\alpha}=D_{1}^{\alpha_{1}} \cdots D_{n}^{\alpha_{n}},|\alpha|=\alpha_{1}+\cdots+\alpha_{n}$, and for convenience when operating with the Fourier transformation, the basic derivatives include the factor $1 / i$, that is, $D_{j}=i^{-1} \partial / \partial x_{j}$, where $i=\sqrt{-1}$. The boundary, $\partial \Omega$, of the domain $\Omega$ is assumed to be $C^{\infty}$.

Let $\alpha_{i j}$ denote the order of $A_{i j}$. If $A_{i j} \equiv 0$, then we set $\alpha_{i j}=-\infty$. Now suppose that we have integers $s_{1}, \ldots, s_{p}, t_{1}, \ldots, t_{p}$ such that the operator $A_{i j}$ has order

$$
\alpha_{i j} \leq s_{i}+t_{j}
$$

where it is to be understood that $A_{i j} \equiv 0$ if $s_{i}+t_{j}<0$. Clearly, any given $s_{i}, t_{j}$ may be replaced by $s_{i}+$ constant, $t_{j}-$ same constant. Then we let $A_{i j}^{\prime}(x, D)$ denote the sum of terms in $A_{i j}(x, D)$ which are exactly of the order $s_{i}+t_{j}$, with lower-order terms replaced by zeros. For arbitrary real $\xi=\left(\xi_{1}, \ldots, \xi_{n}\right) \in \mathbb{R}^{n}$ we define the DN principal part $\pi_{D} \mathcal{A}(x, \xi)$ as the polynomial $p \times p$ matrix

$$
\pi_{D} \mathcal{A}(x, \xi):=\left[A_{i j}^{\prime}(x, \xi)\right]_{p \times p}=\left[\pi_{s_{i}+t_{j}} A_{i j}(x, \xi)\right]_{p \times p} .
$$


Definition 1.1. The operator $\mathcal{A}(x, D)$ is said to be elliptic at $x \in \bar{\Omega}$ if there exist DN numbers $s_{1}, \ldots, s_{p}, t_{1}, \ldots, t_{p}$ such that the characteristic polynomial

$$
\chi(\xi)=\operatorname{det} \pi_{D} \mathcal{A}(x, \xi) \neq 0
$$

for each $0 \neq \xi \in \mathbb{R}^{n}$.

Note that the choice of DN numbers (if they exist) is not unique. Nevertheless, from now on we write $\pi \mathcal{A}$ instead of $\pi_{D} \mathcal{A}$ for the principal part. The characteristic polynomial $\chi(\xi)$ is homogeneous in $\xi$ of degree $m=\sum\left(s_{i}+t_{i}\right)$, that is,

$$
\chi(c \xi)=c^{m} \chi(\xi), \quad c \in \mathbb{R} .
$$

The differential operator $\mathcal{A}(x, D)$ is said to be elliptic on $\bar{\Omega}$ if it is elliptic at each point $x \in \bar{\Omega}$ with a fixed set of DN numbers.

Let $\nu: \partial \Omega \rightarrow \mathrm{T}\left(\mathbb{R}^{n}\right)$ be the inward-pointing unit normal along $\partial \Omega$. For each $x \in \partial \Omega$ there is the direct sum decomposition $\mathrm{T}_{x} \mathbb{R}^{n}=\mathrm{T}_{x}(\partial \Omega) \oplus \operatorname{span}\left(\nu_{x}\right)$, and by passing to the dual, we have $\mathrm{T}_{x}^{*} \mathbb{R}^{n}=\left[\operatorname{span}\left(\nu_{x}\right)\right]^{0} \oplus\left[\mathrm{T}_{x}(\partial \Omega)\right]^{0}$. By identifying the annihilator subspace $\left[\operatorname{span}\left(\nu_{x}\right)\right]^{0}$ with the cotangent space $\mathrm{T}_{x}^{*}(\partial \Omega)$ by means of the restriction map $\left.\xi^{\prime} \mapsto \xi^{\prime}\right|_{\mathrm{T}_{x}(\partial \Omega)}$ we have

$$
\begin{aligned}
\mathrm{T}_{x}^{*} \mathbb{R}^{n} & =\mathrm{T}_{x}^{*}(\partial \Omega) \oplus\left[\mathrm{T}_{x}(\partial \Omega)\right]^{0}, \\
\xi & =\xi^{\prime}+\xi_{n},
\end{aligned}
$$

where $\xi^{\prime} \in \mathrm{T}_{x}^{*}(\partial \Omega)$ is cotangent to $\partial \Omega$ and $\xi_{n}$ is conormal to $\partial \Omega$, i.e. $\left\langle\xi_{n}, v\right\rangle=0$ for all $v \in \mathrm{T}_{x}(\partial \Omega)$.

Now let $n: \partial \Omega \rightarrow \mathrm{T}^{*}\left(\mathbb{R}^{n}\right)$ be the image of $\nu$ by the index-lowering operator $\mathrm{T}\left(\mathbb{R}^{n}\right) \rightarrow \mathrm{T}^{*}\left(\mathbb{R}^{n}\right)$ that maps $\partial / \partial x_{i}$ to $d x_{i}$. Because the space of conormal vectors at a point $x \in \partial \Omega$ is one-dimensional and $n(x) \neq 0$ for each $x$, then every $\xi \in \mathrm{T}_{x}^{*}\left(\mathbb{R}^{n}\right)$ can be written uniquely in the form

$$
\xi=\xi^{\prime}+\xi_{n} \cdot n(x),
$$

where $\xi^{\prime} \in \mathrm{T}_{x}^{*}(\partial \Omega), \xi_{n} \in \mathbb{R}$. This defines a vector bundle isomorphism $\mathrm{T}^{*}\left(\mathbb{R}^{n}\right) \simeq$ $\mathrm{T}^{*}(\partial \Omega) \oplus(\partial \Omega \times \mathbb{R})$, and we are justified in writing, for each $\xi \in \mathrm{T}_{x}^{*} \mathbb{R}^{n}$,

$$
\xi=\left(\xi^{\prime}, \xi_{n}\right)
$$

where $\xi^{\prime} \in \mathrm{T}_{x}^{*}(\partial \Omega), \xi_{n} \in \mathbb{R}$.

The definition of proper ellipticity at a point $x \in \partial \Omega$ on the boundary is as follows. When we substitute $\xi=\left(\xi^{\prime}, \lambda\right)=\xi^{\prime}+\lambda \cdot n(x) \in \mathrm{T}_{x}^{*} \mathbb{R}^{n}$ in the characteristic polynomial $\chi(\xi)=\operatorname{det} \pi \mathcal{A}(x, \xi)$, it is to be understood that the canonical isomorphism $\mathrm{T}_{x}^{*}\left(\mathbb{R}^{n}\right) \simeq \mathbb{R}^{n}$ is taken into account. We may also permit $\lambda$ to be a complex number, and then $\xi \in \mathrm{T}_{x}^{*} \mathbb{R}^{n} \otimes \mathbb{C} \subset \mathbb{C}^{n}$.

Definition 1.2. Let $n \geq 2$. The elliptic operator $\mathcal{A}(x, D)$ is proper at $x \in \partial \Omega$ if for each $0 \neq \xi^{\prime} \in \mathrm{T}_{x}^{*}(\partial \Omega)$ the polynomial in $\lambda \in \mathbb{C}$

$$
P(\lambda)=\chi\left(\xi^{\prime}, \lambda\right)=\operatorname{det} \pi \mathcal{A}\left(x,\left(\xi^{\prime}, \lambda\right)\right)
$$

has as many roots, $r$, in the upper half-plane $\operatorname{Im} \lambda>0$ as in the lower half-plane, $\operatorname{Im} \lambda<0$, counting multiplicities.

Here $\chi=\operatorname{det} \pi \mathcal{A}$ is the characteristic polynomial (2). Since it is a polynomial of degree $m$ in $\left(\xi^{\prime}, \lambda\right)$, then

$$
P(\lambda)=\operatorname{det} \pi \mathcal{A}\left(x,\left(\xi^{\prime}, \lambda\right)\right)=a_{m}(x) \lambda^{m}+\cdots,
$$


with $a_{m}(x)$ independent of $\xi^{\prime}$. By ellipticity with $\xi^{\prime}=0, \lambda=1$, it follows that $a_{m}(x) \neq 0, x \in \partial \Omega$, whence $m$ is the degree of $P(\lambda)$. Also because of ellipticity there are no roots on the real axis, so if $\mathcal{A}$ is properly elliptic we must have $m=2 r$. Hence $m$ is even and the number $r$ in Def. 1.2 is independent of $x \in \partial \Omega$ and $\xi^{\prime} \neq 0$.

Remark. One could try to generalize proper ellipticity and propose that the number $r$ of roots of $P(\lambda)=0$ in the upper half-plane $\operatorname{Im} \lambda>0$ be independent of $\xi^{\prime} \in$ $\mathrm{T}_{x}^{*}(\partial \Omega) \backslash 0$. But by homogeneity of $\operatorname{det} \pi \mathcal{A}(x, \xi)$ with $c=-1$ we have

$$
\operatorname{det} \pi \mathcal{A}\left(x,\left(-\xi^{\prime},-\lambda\right)\right)=(-1)^{m} \operatorname{det} \pi \mathcal{A}\left(x,\left(\xi^{\prime}, \lambda\right)\right), \quad x \in \partial \Omega,
$$

from which it follows that the number, $m-r$, of roots in the lower half-plane $\operatorname{Im} \lambda<0$ is equal to

$$
m-r=r .
$$

Thus we get proper ellipticity.

Definition 1.3. If in the definition of DN ellipticity (Def. 1.1) we can put $s_{1}=$ $\cdots=s_{p}$ and $t_{1}=\cdots=t_{p}$; then the operator $\mathcal{A}$ is said to be homogeneously elliptic. Because $s_{i}$ and $t_{j}$ can be replaced by $s_{i}+$ constant, $t_{j}$-same constant, we can choose $t_{1}=\cdots=t_{p}=0$ (Another possible choice is of course $s_{1}=\cdots=s_{p}=0$ ).

If $t_{1}=\cdots=t_{p}=0$ and $s_{1}=\cdots=s_{p}=\ell$, then writing the homogeneous operator $\mathcal{A}$ in the form

$$
\mathcal{A}(x, D)=\sum_{|\alpha| \leq \ell} A_{\alpha}(x) D^{\alpha}
$$

where the $A_{\alpha}(x)$ are $p \times p$ matrices, we have

$$
\pi \mathcal{A}(x, \xi)=\sum_{|\alpha|=\ell} A_{\alpha}(x) \xi^{\alpha},
$$

and the characteristic polynomial $\chi(\xi)=\operatorname{det} \pi \mathcal{A}(x, \xi)$ has the (maximal) order $\ell \cdot p$.

The boundary, $\partial \Omega$, is a compact $C^{\infty}$ manifold. When dealing with a boundary operator for an elliptic system it is important to be able to work with pseudodifferential operators on $\partial \Omega$; this makes it possible to modify the order of the boundary operator. For any compact $C^{\infty}$ manifold $M$, let $\operatorname{OS}^{m}(M), m \in \mathbb{R}$, be the set of pseudo-differential operators (p.d.o.'s) of order $m$ on $M$ as defined in [Ho, Chap. 18]. For each $m \in \mathbb{R}$, we define the "classical" p.d.o.'s to be those operators, $A \in \operatorname{OS}^{m}(M)$, that have a well-defined principal symbol $\pi A \in C^{\infty}\left(\mathrm{T}^{*}(M) \backslash 0\right)$, positively homogeneous of order $m$; we denote this subset by $\mathrm{OClS}^{m}(M)$. Classical p.d.o.'s are closed under composition of operators. Furthermore, if $A \in \mathrm{OClS}^{m_{1}}(M)$ and $B \in \operatorname{OClS}^{m_{2}}(M)$ then

$$
\pi(A \circ B)=\pi A \cdot \pi B
$$

A Riemannian metric on $M$ gives rise to a positive measure on $M$ and an inner product on $L_{2}(M)$. Classical p.d.o.'s are closed under adjoints, and for $A \in \mathrm{OClS}^{m}(M)$ the principal symbol of the adjoint $A^{*}$ is given by

$$
\pi\left(A^{*}\right)=\overline{\pi A}
$$

the complex conjugate of the principal symbol of $A$. 


\section{Theorem 1.4.}

(i) Any p.d.o. $A \in \mathrm{OS}^{m}(M)$ defines an operator $A: C^{\infty}(M) \rightarrow C^{\infty}(M)$ and an operator

$$
A: W_{2}^{\sigma}(M) \longrightarrow W_{2}^{\sigma-m}(M)
$$

for any $\sigma \in \mathbb{R}$. Here $W_{2}^{\sigma}(M)$ is the Sobolev space of order $\sigma$ on the manifold $M$.

(ii) For any function $h \in C^{\infty}\left(\mathrm{T}^{*}(M) \backslash 0\right)$, positively homogeneous of order $m$,

$$
h(x, c \xi)=c^{m} h(x, \xi), \quad c>0,
$$

there exists a classical p.d.o. $A \in \mathrm{OClS}^{m}(M)$ with principal symbol $\pi A=h$.

The proofs of these results may be found, for instance, in [WRL, Chap. 8].

We turn now to the formulation of boundary value problems for a (properly) elliptic operator $\mathcal{A}$, with DN numbers $s_{1}, \ldots, s_{p}, t_{1}, \ldots, t_{p}$. As usual, we let $D_{n}=$ $i^{-1} \partial / \partial n$, where $n$ is the inward pointing unit (co)normal vector field on $\partial \Omega$. Points on the boundary will be denoted by $y \in \partial \Omega$. Let $r$ be the number of roots of the polynomial $P(\lambda)=\operatorname{det} \pi \mathcal{A}\left(y,\left(\xi^{\prime}, \lambda\right)\right)$ in the upper half-plane $\operatorname{Im} \lambda>0$, i.e. half the order of the characteristic polynomial $\chi$. In addition to the $p$ equations, $\mathcal{A}(x, D) u(x)=f(x)$, in $\Omega$, we consider $r$ boundary conditions

$$
\sum_{j=1}^{p} B_{k j}(y, D) u_{j}(y)=g_{k}(y), \quad k=1, \ldots, r,
$$

that is, $\mathcal{B}(y, D) u(y)=g(y)$, where $\mathcal{B}(y, D)$ is the matrix operator $\left[B_{k j}(y, D)\right]_{r \times p}$. The boundary operators $B_{k j}$ are taken in the form

$$
B_{k j}(y, D)=\sum_{\kappa=0}^{\ell_{k j}} b_{k j}^{\kappa}\left(y, D^{\prime}\right) D_{n}^{\kappa}, \quad y \in \partial \Omega,
$$

where $b_{k j}^{\kappa}\left(y, D^{\prime}\right)$ are (classical) pseudo-differential operators on the manifold $\partial \Omega$. The principal parts are denoted by $\pi b_{k j}^{\kappa}\left(y, \xi^{\prime}\right),\left(y, \xi^{\prime}\right) \in \mathrm{T}^{*}(\partial \Omega) \backslash 0$, and we also write $\beta_{k j}^{\kappa}=\operatorname{ord} b_{k j}^{\kappa}$.

The DN principal part of the boundary operator $\mathcal{B}$ is defined as follows. Let

$$
m_{k j}:=\max _{\kappa} \operatorname{ord}\left(b_{k j}^{\kappa} D_{n}^{\kappa}\right)=\max _{\kappa}\left(\beta_{k j}^{\kappa}+\kappa\right)
$$

(the numbers $m_{k j}$ can be negative and also non-integer, i.e. $m_{k j} \in \mathbb{R}$ ) and then let

$$
m_{k}:=\max _{1 \leq j \leq p}\left(m_{k j}-t_{j}\right), \quad k=1, \ldots, r,
$$

so that $m_{k j} \leq m_{k}+t_{j}$. The $D N$ principal part of the boundary operator $\mathcal{B}(y, D)$ is defined as the $r \times p$ matrix

$$
\pi_{D} \mathcal{B}(y, \xi)=\left[B_{k j}^{\prime}(y, \xi)\right]_{r \times p}, \quad B_{k j}^{\prime}(y, \xi)=\sum_{\kappa}{ }^{\prime} \pi b_{k j}^{\kappa}\left(y, \xi^{\prime}\right) \xi_{n}^{\kappa},
$$

where $\sum_{\kappa}^{\prime}$ denotes the sum over those terms with $\beta_{k j}^{\kappa}+\kappa=m_{k}+t_{j}$. In other words, $B_{k j}^{\prime}(y, \xi)$ consists of the principal parts of the terms in $B_{k j}$ which are just of order $m_{k}+t_{j}$, with the other terms replaced by 0 . As usual, $\xi=\left(\xi^{\prime}, \xi_{n}\right), \xi^{\prime} \in \mathrm{T}_{y}^{*}(\partial \Omega) \backslash 0$ and $\xi_{n}$ is conormal at $y$. From now on we denote the DN principal part by $\pi \mathcal{B}$ rather than $\pi_{D} \mathcal{B}$. 
The operators $b_{k j}^{\kappa}$ can have negative order, $\beta_{k j}^{\kappa}<0$; for some purposes, however, it is convenient to assume that all these orders are non-negative, and this can always be achieved as follows. Let $\Lambda^{q}$ be a pseudo-differential operator with principal symbol $\left|\xi^{\prime}\right|^{q}$, then replace $b_{k j}^{\kappa}$ by $\Lambda^{q} \circ b_{k j}^{\kappa}$ where $q$ is chosen so that $q+\beta_{k j}^{\kappa} \geq 0$ for all $k, j$, that is, we choose

$$
q=-\min _{\kappa, k, j} \beta_{k j}^{\kappa} .
$$

Remark. If $\mathcal{A}$ is homogeneously elliptic (Def. 1.3), then the boundary operators under consideration have order $m_{k}$ in the $k$ th row, $k=1, \ldots, r$.

Our intention now is to formulate the L-condition for elliptic boundary value problem operators $(\mathcal{A}, \mathcal{B})$, but some more preparation is needed.

Consider the decomposition $(4),\left(4^{\prime}\right)$ of the cotangent space $\mathrm{T}_{y_{0}}^{*}(\partial \Omega)$ at the boundary point $y_{0} \in \partial \Omega$, where $y_{0}$ is fixed. We replace $\xi_{n}$ by $\frac{1}{i} \frac{d}{d t}$ and fix $\xi^{\prime} \neq 0$ in the DN principal part of $\mathcal{A}$ to obtain the system of ordinary differential equations (with constant coefficients)

$$
\pi \mathcal{A}\left(y_{0},\left(\xi^{\prime}, \frac{1}{i} \frac{d}{d t}\right)\right) w(t)=0, \quad t>0, \quad \xi^{\prime} \in \mathrm{T}_{y_{0}}^{*}(\partial \Omega) \backslash 0 .
$$

The solutions of this equation are $p$-columns of exponential polynomials of the form $\sum p_{j}(t) e^{i \lambda_{j} t}$, where the $p_{j}$ 's are polynomials in $t$ and the $\lambda_{j}$ 's are eigenvalues of $L(\lambda)$. The solution space $\mathfrak{M}=\mathfrak{M}\left(\xi^{\prime}\right)$ of (12) decomposes directly into

$$
\mathfrak{M}=\mathfrak{M}^{-} \oplus \mathfrak{M}^{+},
$$

where $\mathfrak{M}^{+}$consists of all solutions $w(t)$ with $w(t) \rightarrow 0$ as $t \rightarrow+\infty$. We have $\operatorname{dim} \mathfrak{M}=m, \operatorname{dim} \mathfrak{M}^{+}=r$ and $\operatorname{dim} \mathfrak{M}^{-}=m-r$, and any $w \in \mathfrak{M}^{+}$is a $p$-column of exponential polynomials such that $\operatorname{Im} \lambda_{j}>0$ for all $j$. It is evident that if $w \in \mathfrak{M}^{+}$then $w$ and all its derivatives belong to $L_{2}\left(\mathbb{R}_{+}\right)$, since $\left|e^{i \lambda_{j} t}\right| \leq e^{-c t}$ for some constant $c>0$.

Definition 1.5. The pair of operators

$$
\mathcal{A}(y, D), \mathcal{B}(y, D), \quad y \in \partial \Omega
$$

is said to fulfill the L-condition if for all $y \in \partial \Omega, 0 \neq \xi^{\prime} \in \mathrm{T}_{y}^{*}(\partial \Omega)$, the zero initial value problem

$$
\begin{gathered}
\pi \mathcal{A}\left(y,\left(\xi^{\prime}, \frac{1}{i} \frac{d}{d t}\right)\right) w(t)=0, \quad t>0, \\
\pi \mathcal{B}\left(y,\left(\xi^{\prime}, \frac{1}{i} \frac{d}{d t}\right)\right) w(t)_{\mid t=0}=0
\end{gathered}
$$

has in $\mathfrak{M}^{+}=\mathfrak{M}^{+}\left(\xi^{\prime}\right)$ the unique solution $w(t)=0$.

One could also formulate the L-condition with the second equation replaced by $\pi \mathcal{B}\left(y,\left(\xi^{\prime}, \frac{1}{i} \frac{d}{d t}\right)\right) w(t)_{\mid t=0}=g$ and require that there be a unique solution $w \in \mathfrak{M}^{+}$ for every $g \in \mathbb{C}^{r}$. (See Theorem 3.3(i).)

Now we state the definition of L-ellipticity of a boundary value problem.

Definition 1.6. The boundary value problem

$$
\begin{aligned}
& \mathcal{A}(x, D) u(x)=f(x), \quad x \in \Omega, \\
& \mathcal{B}(y, D) u(y)=g(y), \quad y \in \partial \Omega,
\end{aligned}
$$

is said to be L-elliptic in $\bar{\Omega}$ if: 
(i) the operator $\mathcal{A}(x, D)$ is elliptic for all $x \in \bar{\Omega}$, see Def. 1.1;

(ii) the operator $\mathcal{A}(x, D)$ is properly elliptic for all $x \in \partial \Omega$, see Def. 1.2;

(iii) $\mathcal{A}(y, D), \mathcal{B}(y, D)$ satisfies for all $y \in \partial \Omega$ the L-condition of Def. 1.5.

The second condition is actually superfluous, i.e. it follows from conditions (i) and (iii), for if the mapping

$$
\pi \mathcal{B}\left(y,\left(\xi^{\prime}, \frac{1}{i} \frac{d}{d t}\right)\right)_{\mid t=0}: \mathfrak{M}^{+}\left(\xi^{\prime}\right) \longrightarrow \mathbb{C}^{r},
$$

is bijective for all $\xi^{\prime} \neq 0$, then the dimension of $\mathfrak{M}^{+}\left(\xi^{\prime}\right)$ must be independent of $\xi^{\prime}$, i.e. the number of roots of $P(\lambda)=\operatorname{det} \pi \mathcal{A}\left(y,\left(\xi^{\prime}, \lambda\right)\right)=0$ in $\operatorname{Im} \lambda>0$ is independent of $\xi^{\prime}$ (equal to the number of boundary conditions), and proper ellipticity of $\mathcal{A}$ follows as in the Remark after Def. 1.2.

Recall that for any integer $\ell=0,1,2, \ldots$, the Sobolev space $W_{2}^{\ell}(\Omega)$ is defined as the set of all functions $u \in L_{2}(\Omega)$ for which the distributional derivatives $D^{\alpha} u$ (or weak derivatives) belong to $L_{2}(\Omega)$ for $|\alpha| \leq \ell$ :

$$
W_{2}^{\ell}(\Omega)=\left\{u \in L_{2}(\Omega) ; D^{\alpha} u \in L_{2}(\Omega) \text { for }|\alpha| \leq \ell\right\} .
$$

We introduce a scalar product on $W_{2}^{\ell}(\Omega)$ by means of

$$
(u, v)_{\ell}:=\sum_{|\alpha| \leq \ell} \int_{\Omega} D^{\alpha} u(x) \cdot \overline{D^{\alpha} v(x)} d x
$$

where, as usual, functions which are equal almost everywhere are identified. This inner product makes $W_{2}^{\ell}(\Omega)$ into a Hilbert space. The corresponding norm is also denoted with a subscript $\ell$, i.e. $\|u\|_{\ell}^{2}=(u, u)_{\ell}$. (One can define the Sobolev spaces on $\Omega$ for any non-negative real number $\ell$, but they will not be needed here.) On the boundary $\partial \Omega$ we will also work with the Sobolev spaces $W_{2}^{\sigma}(\partial \Omega)$ for any real number $\sigma$. The definition of these spaces requires the use of a partition of unity subordinate to an atlas on the manifold $\partial \Omega$. For details on Sobolev spaces see [Ad], [Wl].

Before continuing, note the following fact about the weights $s_{1}, \ldots, s_{p}, t_{1}, \ldots, t_{p}$ in the definition of ellipticity:

$$
\max _{i} s_{i} \geq-t_{j}, \quad \text { for all } j .
$$

Otherwise, there would exist $j_{0}$ such that $s_{i}+t_{j_{0}}<0$ for all $i$, so that $A_{i j_{0}}(x, D) \equiv 0$, in contradiction to ellipticity. Replacing the weights $s_{i}$ and $t_{j}$ with $s_{i}-$ const and $t_{j}+$ const, we may assume that the weights are normalized as follows:

$$
s_{i} \leq 0, \quad t_{j} \geq 0, \quad \text { for all } i, j, \quad \text { and } \max _{i} s_{i}=0 .
$$

Finally, here is the formulation of the Fredholm property for L-elliptic systems. A Fredholm operator is a bounded linear operator whose kernel has finite dimension, $\alpha$, and whose image has finite codimension, $\beta$; the index of this operator is defined to be $\alpha-\beta$. We define the Hilbert spaces

$$
\begin{gathered}
\mathcal{W}^{\ell+\mathbf{t}}(\Omega)=W_{2}^{\ell+t_{1}}(\Omega) \times \cdots \times W_{2}^{\ell+t_{p}}(\Omega), \\
\mathcal{W}^{\ell-\mathbf{s}}(\Omega)=W_{2}^{\ell-s_{1}}(\Omega) \times \cdots \times W_{2}^{\ell-s_{p}}(\Omega), \\
\mathcal{W}^{\ell-\mathbf{m}-1 / 2}(\partial \Omega)=W_{2}^{\ell-m_{1}}(\partial \Omega) \times \cdots \times W_{2}^{\ell-m_{r}}(\partial \Omega),
\end{gathered}
$$


with the natural inner products induced by the direct sums. For instance, the norm for $u \in \mathcal{W}^{\ell+\mathbf{t}}(\Omega)$ is $\|u\|_{\ell+\mathbf{t}}^{2}=\sum_{j=1}^{p}\left\|u_{j}\right\|_{\ell+t_{j}}^{2}$, and similarly for $\mathcal{W}^{\ell-\mathbf{s}}(\Omega)$ and $\mathcal{W}^{\ell-\mathbf{m}-1 / 2}(\partial \Omega)$. Here $\ell$ is an integer such that

$$
\ell \geq \ell_{0}:=\max \left\{\max _{k} m_{k}+q+1, \max _{i} s_{i}\right\},
$$

and $q=-\min _{\kappa, k, j} \beta_{k j}^{\kappa}$; see (11). With the normalization (15) we have $\ell_{0} \geq 0$. Corresponding to the boundary value problem (18) we have the operator

$$
\mathfrak{A}=(\mathcal{A}(x, D), \mathcal{B}(y, D)): \mathcal{W}^{\ell+\mathbf{t}}(\Omega) \longrightarrow \mathcal{W}^{\ell-\mathbf{s}}(\Omega) \times \mathcal{W}^{\ell-\mathbf{m}-1 / 2}(\partial \Omega),
$$

which is a continuous linear operator, i.e.

$$
\|\mathcal{A} u\|_{\ell-\mathbf{s}}+\|\mathcal{B} u\|_{\ell-\mathbf{m}-1 / 2} \leq \text { const. }\|u\|_{\ell+\mathbf{t}},
$$

since ord $A_{i j} \leq s_{i}+t_{j}$ and ord $B_{k j} \leq m_{k}+t_{j}$.

Theorem 1.7. Let $\Omega$ be a bounded domain in $\mathbb{R}^{n}$ with a $C^{\infty}$ boundary $\partial \Omega$. For the boundary value problem

$$
\begin{aligned}
& \mathcal{A}(x, D) u(x)=f(x), \quad x \in \Omega, \\
& \mathcal{B}(y, D) u(y)=g(y), \quad y \in \partial \Omega
\end{aligned}
$$

the following conditions are equivalent:

(a) the boundary value problem (18) is L-elliptic (Def. 1.6);

(b) the operator (17) is Fredholm;

(c) for all $u \in \mathcal{W}^{\ell+\mathbf{t}}(\Omega), \ell \geq \ell_{0}$, there is the a priori estimate

$$
\|u\|_{\ell+\mathbf{t}} \leq c\left[\|\mathcal{A} u\|_{\ell-\mathbf{s}}+\|\mathcal{B} u\|_{\ell-\mathbf{m}-1 / 2}+\|u\|_{\ell+\mathbf{t}-1}\right] .
$$

For the proof, see [WRL, Chap. 9]. It turns out that if (b) holds for just one $\ell \geq \ell_{0}$ then it holds for every $\ell \geq \ell_{0}$. The same is true for (c).

Remark. Note that

$$
\ell_{0}+t_{j} \geq 1, \quad \text { for all } j .
$$

This is clearly true when $\max _{k} m_{k}+q \geq 0$, so it remains to consider the case when $\max _{k} m_{k}+q<0$. By definition of $m_{k}$ and $q$, we then have

$$
m_{k j}-t_{j}<\beta_{k j}^{\kappa}
$$

for all $\kappa=0, \ldots, \ell_{k j}, j=1, \ldots, \ell$ and $k=1, \ldots, r$. The definition of $m_{k j}$ implies that $\beta_{k j}^{\kappa}+\kappa-t_{j}<\beta_{k j}^{\kappa}$ for all $j, k$ and $\kappa$. Hence $t_{j}>0$, and $t_{j}$ being an integer we have $t_{j} \geq 1$. This proves (20) since $\ell_{0} \geq 0$.

Note. The inequality $(20)$ ensures that $\mathcal{W}^{\ell+\mathbf{t}-1}(\Omega) \subset L_{2}(\Omega) \times \cdots \times L_{2}(\Omega)$ when $\ell \geq \ell_{0}$.

The next section develops a spectral theory of matrix polynomials which makes it possible to reformulate the L-condition in various equivalent algebraic forms. 


\section{SPECTRAL TRIPLES FOR MATRIX POLYNOMials}

For any finite dimensional vector spaces $\mathfrak{M}, \mathfrak{N}$, let $\mathcal{L}(\mathfrak{M}, \mathfrak{N})$ denote the set of linear maps from $\mathfrak{M}$ to $\mathfrak{N}$. A triple of operators $(X, T, Y)$ is called an admissible triple if $X \in \mathcal{L}\left(\mathfrak{M}, \mathbb{C}^{p}\right), T \in \mathcal{L}(\mathfrak{M})=\mathcal{L}(\mathfrak{M}, \mathfrak{M})$ and $Y \in \mathcal{L}\left(\mathbb{C}^{p}, \mathfrak{M}\right)$. The vector space $\mathfrak{M}$ is called the base space of the admissible triple. Two admissible triples, $(X, T, Y)$ and $\left(X^{\prime}, T^{\prime}, Y^{\prime}\right)$, are called similar if there exists an invertible operator $M \in \mathcal{L}\left(\mathfrak{M}^{\prime}, \mathfrak{M}\right)$ such that

$$
X^{\prime}=X M, \quad T^{\prime}=M^{-1} T M, \quad \text { and } \quad Y^{\prime}=M^{-1} Y
$$

The admissible pair $(X, T)$ is referred to as a right admissible pair, while $(T, Y)$ is called a left admissible pair. If $S_{j} \in \mathcal{L}\left(\mathfrak{M}, \mathfrak{N}_{j}\right), j=1, \ldots, n$, we define

$$
\operatorname{col}\left(S_{j}\right)_{j=1}^{n}=\left(\begin{array}{c}
S_{1} \\
\vdots \\
S_{n}
\end{array}\right) \in \mathcal{L}\left(\mathfrak{M}, \mathfrak{N}_{1} \oplus \cdots \oplus \mathfrak{N}_{n}\right)
$$

Similarly, if $T_{j} \in \mathcal{L}\left(\mathfrak{M}_{j}, \mathfrak{N}\right), j=1, \ldots, n$, we define

$$
\operatorname{row}\left(T_{j}\right)_{j=1}^{n}=\left[\begin{array}{lll}
T_{1} & \ldots & T_{n}
\end{array}\right] \in \mathcal{L}\left(\mathfrak{M}_{1} \oplus \cdots \oplus \mathfrak{M}_{n}, \mathfrak{N}\right)
$$

and if $W_{j} \in \mathcal{L}\left(\mathfrak{M}_{j}, \mathfrak{N}_{j}\right), j=1, \ldots, n$, we define

$$
\operatorname{diag}\left(W_{j}\right)_{j=1}^{n}=\left(\begin{array}{ccc}
W_{1} & & 0 \\
& \ddots & \\
0 & & W_{n}
\end{array}\right) \in \mathcal{L}\left(\mathfrak{M}_{1} \oplus \cdots \oplus \mathfrak{M}_{n}, \mathfrak{N}_{1} \oplus \cdots \oplus \mathfrak{N}_{n}\right) .
$$

Let $L(\lambda)=\sum_{j=0}^{\ell} A_{j} \lambda^{j}$ be a $p \times p$ matrix polynomial. A complex number $\lambda_{0}$ is called an eigenvalue of $L(\lambda)$ if

$$
\operatorname{det} L\left(\lambda_{0}\right)=0
$$

and the set of all eigenvalues is called the spectrum of $L(\lambda)$, denoted $\operatorname{sp}(L)$, i.e.

$$
\operatorname{sp}(L)=\{\lambda \in \mathbb{C} ; \operatorname{det} L(\lambda)=0\} .
$$

Now let $\gamma$ be a simple, closed (rectifiable) contour not intersecting $\operatorname{sp}(L)$. Also let $G$ denote the region inside $\gamma$. In part (i) of the following definition, the notation $\operatorname{sp}\left(T_{+}\right)$of course refers to the spectrum of $I \lambda-T_{+}$.

Definition 2.1. A $\gamma$-spectral triple for $L(\lambda)$ is defined to be an admissible triple $\left(X_{+}, T_{+}, Y_{+}\right)$with the following properties:

(i) $\operatorname{sp}\left(T_{+}\right) \subset G$ (i.e. inside $\left.\gamma\right)$,

(ii) $L^{-1}(\lambda)-X_{+}\left(I \lambda-T_{+}\right)^{-1} Y_{+}$has an analytic continuation in $G$,

(iii) $\operatorname{col}\left(X_{+} T_{+}^{j}\right)_{j=0}^{\ell-1}$ is injective,

(iv) $\operatorname{row}\left(T_{+}^{j} Y_{+}\right)_{j=0}^{\ell-1}$ is surjective.

Also, we say that $\left(X_{+}, T_{+}\right)$is a (right) $\gamma$-spectral pair for $L(\lambda)$ and $\left(T_{+}, Y_{+}\right)$is a left $\gamma$-spectral pair for $L(\lambda)$.

If the contour $\gamma$ is chosen so that all of $\operatorname{sp}(L)$ lies inside $\gamma$, then we obtain a triple $(X, T, Y)$ which we call a finite spectral triple (i.e. for the finite complex plane). If $\gamma$ is chosen so that it surrounds just one eigenvalue $\lambda_{0} \in \operatorname{sp}(L)$, then we obtain a triple $\left(X_{0}, T_{0}, Y_{0}\right)$ which we call a spectral triple at $\lambda_{0}$. If $T_{0}=J_{0}$ is a Jordan matrix, then we refer to $\left(X_{0}, J_{0}, Y_{0}\right)$ as a Jordan triple at $\lambda_{0}$. 
Proposition 2.2. Property (ii) of Def. 2.1 can be replaced by

$$
\frac{1}{2 \pi i} \int_{\gamma} \lambda^{j} L^{-1}(\lambda) d \lambda=X_{+} T_{+}^{j} Y_{+}, \quad j=0,1, \ldots
$$

Proof. The property (ii) holds if and only if

$$
\frac{1}{2 \pi i} \int_{\gamma} \lambda^{j}\left\{L^{-1}(\lambda)-X_{+}\left(I \lambda-T_{+}\right)^{-1} Y_{+}\right\} d \lambda=0, \quad j=0,1, \ldots
$$

Since $\operatorname{sp}\left(T_{+}\right) \subset G$, this is equivalent to $\left(\mathrm{ii}^{\prime}\right)$.

Proposition 2.3. If $\left(X_{+}, T_{+}\right)$is a $\gamma$-spectral pair for $L(\lambda)$, then

$$
\sum_{j=0}^{\ell} A_{j} X_{+} T_{+}^{j}=0
$$

Proof. By definition, there exists an operator $Y_{+}$such that $\left(X_{+}, T_{+}, Y_{+}\right)$is a $\gamma$ spectral triple for $L(\lambda)$. Then property (ii') implies that

$$
\sum_{j=0}^{\ell} A_{j} X_{+} T_{+}^{j} \cdot T_{+}^{k} Y_{+}=\frac{1}{2 \pi i} \int_{\gamma} \sum_{j=0}^{\ell} A_{j} \lambda^{j} \cdot \lambda^{k} L^{-1}(\lambda) d \lambda=0
$$

for $k=0,1, \ldots$ and, by property (iv), it follows that $\sum_{j=0}^{\ell} A_{j} X_{+} T_{+}^{j}=0$.

Remark 2.4. If $\left(X_{+}, T_{+}, Y_{+}\right)$is a $\gamma$-spectral triple for $L(\lambda)$, then $\left(Y_{+}^{T}, T_{+}^{T}, X_{+}^{T}\right)$ is a $\gamma$-spectral triple for $L^{T}(\lambda)$, where $L^{T}(\lambda)=\sum A_{j}^{T} \lambda^{j}$ is the matrix polynomial obtained by transposing the coefficients of $L(\lambda)$. Analogous to Prop. 2.3 one can show that if $\left(T_{+}, Y_{+}\right)$is a left $\gamma$-spectral pair for $L(\lambda)$, then

$$
\sum_{j=0}^{\ell} T_{+}^{j} Y_{+} A_{j}=0
$$

In order to prove the existence of $\gamma$-spectral triples, we need the fact that every solution of the homogeneous differential equation $L(d / d t) u(t)=0$ can be expressed in the form

$$
u(t)=\frac{1}{2 \pi i} \int_{\gamma} e^{t \lambda} L^{-1}(\lambda) f(\lambda) d \lambda
$$

for some $\mathbb{C}^{p}$-valued polynomial $f(\lambda)$ of degree $\leq \ell-1$. In fact, the following result is true. Let $\mathfrak{M}_{L}$ denote the set of solutions $u \in C^{\infty}\left(\mathbb{R}, \mathbb{C}^{p}\right)$ of the equation $L(d / d t) u(t)=0$. Every $u \in \mathfrak{M}_{L}$ can be written in the form

$$
u(t)=\sum p_{i}(t) e^{t \lambda_{i}}
$$

where the $p_{i}$ are $\mathbb{C}^{p}$-valued polynomials, and the complex numbers $\lambda_{i}$ are eigenvalues of $L(\lambda)$, i.e. roots of the polynomial equation $\operatorname{det} L(\lambda)=0$. It is well known that the dimension of $\mathfrak{M}_{L}$ is equal to the degree, $\alpha$, of $\operatorname{det} L(\lambda)$.

Let $\mathfrak{M}_{L}^{+}$denote the subspace of $\mathfrak{M}_{L}$ consisting of solutions of the form (2) such that the eigenvalues $\lambda_{i}$ lie inside $\gamma$.

Proposition 2.5. Every $u \in \mathfrak{M}_{L}^{+}$has the representation

$$
u(t)=\frac{1}{2 \pi i} \int_{\gamma} e^{t \lambda} L^{-1}(\lambda) \sum_{j=0}^{\ell-1} L_{j+1}(\lambda) u^{(j)}(0) d \lambda,
$$


where $L_{j}(\lambda)=A_{j}+A_{j+1} \lambda+\cdots+A_{\ell} \lambda^{\ell-j}, j=0, \ldots, \ell$. This expresses $u$ in terms of its Cauchy data at $t=0$.

The proof is quite simple when $L(\lambda)$ has invertible leading coefficient, $A_{\ell}$. The general case requires somewhat more work, and the reader is referred to [WRL, Chap. 1] for the details.

Now we prove existence of $\gamma$-spectral triples. It is also possible to show that they are unique up to similarity; see p. 31 in [WRL].

Theorem 2.6. Let $\gamma$ be a simple, closed contour not intersecting $\operatorname{sp}(L)$. Then there exists a $\gamma$-spectral triple for $L(\lambda)$.

Proof. We define an admissible triple $\left(X_{+}, T_{+}, Y_{+}\right)$with base space $\mathfrak{M}_{L}^{+}$as follows:

$$
\begin{aligned}
X_{+} u & =u(0), \\
T_{+} u & =\frac{d u}{d t}, \\
\left(Y_{+} x\right)(t) & =\frac{1}{2 \pi i} \int_{\gamma} e^{t \lambda} L^{-1}(\lambda) x d \lambda .
\end{aligned}
$$

(Note that if $u \in \mathfrak{M}_{L}^{+}$then $d u / d t \in \mathfrak{M}_{L}^{+}$.) We will show that $\left(X_{+}, T_{+}, Y_{+}\right)$satisfies properties (i), (ii'), (iii) and (iv) of Def. 2.1.

Let $\lambda_{0} \notin \bar{G}$. Then define $S \in \mathcal{L}\left(\mathfrak{M}_{L}^{+}\right)$as follows:

$$
(S u)(t)=\frac{1}{2 \pi i} \int_{\gamma} e^{t \lambda} L^{-1}(\lambda) f(\lambda)\left(\lambda_{0}-\lambda\right)^{-1} d \lambda,
$$

where $f$ determines $u$ as in (1). Note that if $\int_{\gamma} e^{t \lambda} L^{-1}(\lambda) f(\lambda) d \lambda=0$ for all $t$, then $L^{-1}(\lambda) f(\lambda)$ has an analytic continuation inside $\gamma$; consequently, the expression defined by the right hand side of (4) is also 0 , and $S$ is well-defined. One can now verify immediately that $\left(I \lambda_{0}-T_{+}\right) S u=u$ and $S\left(I \lambda_{0}-T_{+}\right) u=u$ for all $u \in \mathfrak{M}_{L}^{+}$. Hence $\left(I \lambda_{0}-T_{+}\right)^{-1}$ exists and $\operatorname{sp}\left(T_{+}\right) \subset \bar{G}$. In fact, $\operatorname{sp}\left(T_{+}\right) \subset G$, since there exists a contour $\gamma^{\prime}$ contained in $G$ such that the part of $\operatorname{sp}(L)$ lying inside $\gamma^{\prime}$ is the same as that inside $\gamma$, and we can replace $\gamma$ with $\gamma^{\prime}$ in the formula (4). This proves (i).

The fact that (ii') of Prop. 2.2 holds is an immediate consequence of the definition of $\left(X_{+}, T_{+}, Y_{+}\right)$.

Now recall that for every $u \in \mathfrak{M}_{L}^{+}$the identity (3) holds. Since $u^{(j)}(0)=X_{+} T_{+}^{j} u$, the injectivity of $\operatorname{col}\left(X_{+} T_{+}^{j}\right)_{j=0}^{\ell-1}$ follows from (3). This proves (iii).

Finally, to prove (iv), note that (3) can be written in the form

$$
u(t)=\frac{1}{2 \pi i} \int_{\gamma} e^{t \lambda} L^{-1}(\lambda)\left[\begin{array}{lll}
I & \ldots & \lambda^{\ell-1} I
\end{array}\right] d \lambda \cdot \mathcal{Z} \cdot \mathcal{U},
$$

where $\mathcal{U}=\operatorname{col}\left(u^{(j)}(0)\right)_{j=0}^{\ell-1}$, the Cauchy data of $u$ at $t=0$, and

$$
\mathcal{Z}=\left(\begin{array}{cccc}
A_{1} & A_{2} & \ldots & A_{\ell} \\
A_{2} & & & 0 \\
\vdots & & & \vdots \\
A_{\ell} & 0 & \ldots & 0
\end{array}\right)
$$

a $p \ell \times p \ell$ matrix (with $A_{1}, A_{2}, \ldots, A_{\ell}$ along the reverse diagonals and zeros below the diagonal of $A_{\ell}$ 's). By the definition of $T_{+}$and $Y_{+}$above, we obtain

$$
u=\operatorname{row}\left(T_{+}^{j} Y_{+}\right)_{j=0}^{\ell-1} \cdot \mathcal{Z} \cdot \operatorname{col}\left(X_{+} T_{+}^{k}\right)_{k=0}^{\ell-1} u,
$$


so that $\operatorname{row}\left(T_{+}^{j} Y_{+}\right)_{j=0}^{\ell-1}$ is surjective. This completes the proof of the theorem.

Having proved existence of spectral triples, we now turn to develop various properties of spectral triples. We would like to show that the spectrum of $T_{+}$coincides with the part of the spectrum of $L(\lambda)$ inside $\gamma$.

Lemma 2.7. Let $L(\lambda)=\sum_{j=0}^{\ell} A_{j} \lambda^{j}$ be a matrix polynomial of degree $\ell$, and define

$$
L_{j}(\lambda)=A_{j}+A_{j+1} \lambda+\cdots+A_{\ell} \lambda^{\ell-j}, \quad j=0, \ldots, \ell .
$$

If $(X, T)$ is an admissible pair such that $\sum_{j=0}^{\ell} A_{j} X T^{j}=0$, then

$$
L(\lambda) \cdot X=\sum_{j=0}^{\ell-1} L_{j+1}(\lambda) \cdot X T^{j}(I \lambda-T) .
$$

Proof. Since $\lambda L_{j+1}(\lambda)=L_{j}(\lambda)-A_{j}$, then

$$
\begin{aligned}
\sum_{j=0}^{\ell-1} L_{j+1}(\lambda) X T^{j}(I \lambda-T) & =\sum_{j=0}^{\ell-1}\left(L_{j}(\lambda)-A_{j}\right) X T^{j}-\sum_{j=0}^{\ell-1} L_{j+1}(\lambda) X T^{j+1} \\
& =L_{0}(\lambda) X-\sum_{j=0}^{\ell} A_{j} X T^{j} \\
& =L(\lambda) X .
\end{aligned}
$$

Proposition 2.8. If $\left(X_{+}, T_{+}, Y_{+}\right)$is a $\gamma$-spectral triple for $L(\lambda)$, then $\operatorname{sp}\left(T_{+}\right)=$ $\operatorname{sp}(L) \cap G$.

Proof. By definition, we have $\operatorname{sp}\left(T_{+}\right) \subset G$. Now if $\lambda_{0} \in \operatorname{sp}\left(T_{+}\right)$, there exists $v_{0} \neq 0$ such that $T_{+} v_{0}=\lambda_{0} v_{0}$. Then, by Prop. 2.3,

$$
0=\sum_{j=0}^{\ell} A_{j} X_{+} T_{+}^{j} v_{0}=\sum_{j=0}^{\ell} A_{j} \lambda_{0}^{j} X_{+} v_{0}=L\left(\lambda_{0}\right) X_{+} v_{0} .
$$

Note that $X_{+} v_{0} \neq 0$; otherwise if $X_{+} v_{0}=0$ then $X_{+} T_{+}^{j} v_{0}=\lambda_{0}^{j} X_{+} v_{0}=0$, for all $j=0,1, \ldots$, and, due to (iii) of Def. 2.1, we would have $v_{0}=0$. Hence $\lambda_{0} \in \operatorname{sp}(L)$ and it follows that $\operatorname{sp}\left(T_{+}\right) \subset \operatorname{sp}(L) \cap G$.

Next we show that if $\lambda \in G \backslash \operatorname{sp}(L)$ then $\lambda \notin \operatorname{sp}\left(T_{+}\right)$. Since

$$
M(\lambda):=L^{-1}(\lambda)-X_{+}\left(I \lambda-T_{+}\right)^{-1} Y_{+}
$$

is analytic in $G$, it follows that $L^{-1}(\lambda)$ has an analytic continuation to $G \backslash \operatorname{sp}\left(T_{+}\right)$, which is $N(\lambda):=M(\lambda)+X_{+}\left(I \lambda-T_{+}\right)^{-1} Y_{+}$. If $\lambda \in G \backslash \operatorname{sp}(L)$ then

$$
\begin{aligned}
I & =L(\lambda) N(\lambda) \\
& =L(\lambda) M(\lambda)+L(\lambda) X_{+}\left(I \lambda-T_{+}\right)^{-1} Y_{+} \\
& =L(\lambda) M(\lambda)+\sum_{j=0}^{\ell-1} L_{j+1}(\lambda) X_{+} T_{+}^{j} Y_{+},
\end{aligned}
$$

where the latter equality holds by Lemma 2.7. But $G$ is connected, so (7) holds for all $\lambda \in G$. Another application of Lemma 2.7 implies $I=L(\lambda) N(\lambda)$ for all $\lambda \in G \backslash \operatorname{sp}\left(T_{+}\right)$. Similarly, $N(\lambda) L(\lambda)=I$ there. Hence $(G \backslash \operatorname{sp}(L)) \cap \operatorname{sp}\left(T_{+}\right)=\emptyset$, and it follows that $\operatorname{sp}\left(T_{+}\right)=\operatorname{sp}(L) \cap G$. 
The Calderón projector. For any $u \in \mathfrak{M}_{L}$, the column vector $\mathcal{U} \in \mathbb{C}^{p \ell}$ defined by

$$
\mathcal{U}=\operatorname{col}\left(u^{(j)}(0)\right)_{j=0}^{\ell-1}
$$

is the Cauchy data (or initial conditions) of $u$ at $t=0$. Recall that every $u \in \mathfrak{M}_{L}^{+}$ has the representation

$$
u(t)=\frac{1}{2 \pi i} \int_{\gamma} e^{t \lambda} L^{-1}(\lambda)\left[L_{1}(\lambda) \quad \ldots \quad L_{\ell}(\lambda)\right] d \lambda \cdot \mathcal{U}
$$

(see Prop. 2.5); then by taking initial conditions on the left-hand side of this equation we obtain $\mathcal{U}=P_{\gamma} \cdot \mathcal{U}$, where

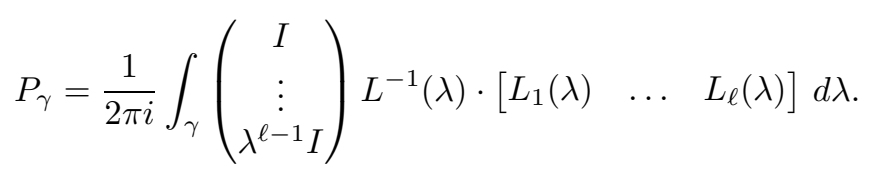

The following theorem shows that $P_{\gamma}$ is a projector, which we call the Calderón projector because of the reference to Calderón in [Se].

Theorem 2.9. $P_{\gamma}$ is a projector in $\mathbb{C}^{p \ell}$. The image of $P_{\gamma}$ is equal to the set of all Cauchy data, $\mathcal{U}$, of functions $u \in \mathfrak{M}_{L}^{+}$.

Proof. In view of the equation $\mathcal{U}=P_{\gamma} \cdot \mathcal{U}$, the set of Cauchy data of functions $u \in \mathfrak{M}_{L}^{+}$is contained in the image of $P_{\gamma}$. On the other hand, the image of $P_{\gamma}$ is contained in the set of Cauchy data, for if $c=\left[\begin{array}{lll}c_{0} & \ldots & c_{\ell-1}\end{array}\right] \in \mathbb{C}^{p \ell}$ let

$$
u(t)=\frac{1}{2 \pi i} \int_{\gamma} e^{t \lambda} L^{-1}(\lambda) \sum_{j=0}^{\ell-1} L_{j+1}(\lambda) c_{j} d \lambda .
$$

Then $u \in \mathfrak{M}_{L}^{+}$and its Cauchy data is $\mathcal{U}=\operatorname{col}\left(u^{(j)}(0)\right)_{j=0}^{\ell-1}=P_{\gamma} c$. The fact that $P_{\gamma}$ is a projector is now clear, for the equation $\mathcal{U}=P_{\gamma} \cdot \mathcal{U}$ implies that $P_{\gamma}$ is the identity on its image.

Corollary 2.10. Let $\left(X_{+}, T_{+}\right)$be a $\gamma$-spectral pair of $L(\lambda)$ (Def. 2.1). Then $P_{\gamma}$ and $\operatorname{col}\left(X_{+} T_{+}^{j}\right)_{j=0}^{\ell-1}$ have the same image. Hence every $u \in \mathfrak{M}_{L}^{+}$has a representation

$$
u(t)=X_{+} e^{t T_{+}} c
$$

for a unique $c$ in the base space of $\left(X_{+}, T_{+}\right)$.

Proof. Let $\left(X_{+}, T_{+}, Y_{+}\right)$be a $\gamma$-spectral triple for $L(\lambda)$ with base space denoted by $\mathfrak{M}_{+}$. By virtue of (ii') in Prop. 2.2, we have

$$
P_{\gamma}=\operatorname{col}\left(X_{+} T_{+}^{j}\right)_{j=0}^{\ell-1} \cdot \operatorname{row}\left(T_{+}^{j} Y_{+}\right)_{j=0}^{\ell-1} \cdot \mathcal{Z}
$$

where $\mathcal{Z}$ is defined by (5). It follows that the image of $P_{\gamma}$ is contained in that of $\operatorname{col}\left(X_{+} T_{+}^{j}\right)_{j=0}^{\ell-1}$. On the other hand, for any $c \in \mathfrak{M}_{+}$the Cauchy data $\mathcal{U}$ of the function $u \in \mathfrak{M}_{L}^{+}$defined by

$$
u(t)=X_{+} e^{t T_{+}} c
$$

satisfies $\mathcal{U}=\operatorname{col}\left(X_{+} T_{+}^{j}\right)_{j=0}^{\ell-1} c$. This proves the first part of the corollary, i.e. $P_{\gamma}$ and $\operatorname{col}\left(X_{+} T_{+}^{j}\right)_{j=0}^{\ell-1}$ have the same image. Functions of the form $\left(^{*}\right)$ certainly belong to $\mathfrak{M}_{L}^{+}$, by virtue of Prop. 2.3 and the fact that $\operatorname{sp}\left(T_{+}\right) \subset$ inside $\gamma$. Conversely, if 
$u \in \mathfrak{M}_{L}^{+}$, then by Theorem 2.9 its Cauchy data, $\mathcal{U}$, is equal to $P_{\gamma} c$ for some $c \in \mathbb{C}^{p \ell}$. Since $u$ and the function $\tilde{u}(t)=X_{+} e^{t T_{+}} c$ have the same initial conditions, i.e.

$$
u^{(j)}(0)=\tilde{u}^{(j)}(0), \quad j=0,1, \ldots, \ell-1,
$$

it follows from $\left(3^{\prime}\right)$ that $u=\tilde{u}$. Hence every $u \in \mathfrak{M}_{L}^{+}$has a representation $\left(^{*}\right)$, and $c$ is unique due to the injectivity of $\operatorname{col}\left(X_{+} T_{+}^{j}\right)_{j=0}^{\ell-1}$.

Let $r$ denote the number of roots of $\operatorname{det} L(\lambda)=0$ inside $\gamma$, multiplicities counted. Since $r$ is the dimension of $\mathfrak{M}_{L}^{+}$, we obtain the following result.

Corollary 2.11. The base space of any $\gamma$-spectral pair of $L(\lambda)$ has dimension $r$.

Proof. This follows from the uniqueness of $c$ in Corollary 2.10 and the fact that $r$ is the dimension of $\mathfrak{M}_{L}^{+}$.

The next corollary will be used frequently in later sections.

Corollary 2.12. If $\left(X_{+}, T_{+}, Y_{+}\right)$is a $\gamma$-spectral triple of $L(\lambda)$, then

$$
\operatorname{row}\left(T_{+}^{j} Y_{+}\right)_{j=0}^{\ell-1} \cdot \mathcal{Z} \cdot \operatorname{col}\left(X_{+} T_{+}^{j}\right)_{j=0}^{\ell-1}=I_{\mathfrak{M}_{+}} .
$$

(Thus, $\operatorname{row}\left(T_{+}^{j} Y_{+}\right)_{j=0}^{\ell-1} \cdot \mathcal{Z}$ is a left inverse of $\operatorname{col}\left(X_{+} T_{+}^{j}\right)_{j=0}^{\ell-1}$, or, stated another way, $\mathcal{Z} \cdot \operatorname{col}\left(X_{+} T_{+}^{j}\right)_{j=0}^{\ell-1}$ is a right inverse of $\left.\operatorname{row}\left(T_{+}^{j} Y_{+}\right)_{j=0}^{\ell-1} \cdot\right)$

Proof. Since $P_{\gamma} \cdot \operatorname{col}\left(X_{+} T_{+}^{j}\right)_{j=0}^{\ell-1}=\operatorname{col}\left(X_{+} T_{+}^{j}\right)_{j=0}^{\ell-1}$, the result follows by virtue of (9) and the injectivity of $\operatorname{col}\left(X_{+} T_{+}^{j}\right)_{j=0}^{\ell-1}$.

The "left" Calderón projector is

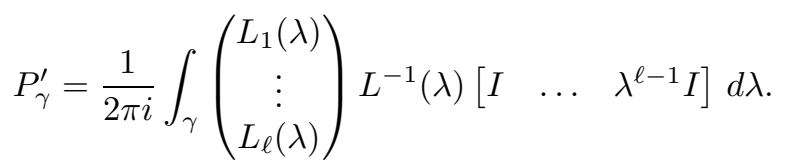

Note that $P_{\gamma}^{\prime}$ is just the transpose of the Calderón projector for the transposed matrix polynomial $L^{T}(\lambda)$. In the following theorem $\operatorname{ker} P_{\gamma}^{\prime}$ denotes the kernel of the operator $P_{\gamma}^{\prime}$.

Theorem 2.13. $P_{\gamma}^{\prime}$ is a projector in $\mathbb{C}^{p \ell}$. Also, if $\left(T_{+}, Y_{+}\right)$is any left $\gamma$-spectral pair of $L(\lambda)$ (see Def. 2.1), then $\operatorname{ker} P_{\gamma}^{\prime}=\operatorname{ker} \operatorname{row}\left(T_{+}^{j} Y_{+}\right)_{j=0}^{\ell-1}$.

Proof. Note that

$$
P_{\gamma}^{\prime}=\mathcal{Z} \cdot\left(\begin{array}{c}
X_{+} \\
\vdots \\
X_{+} T_{+}^{\ell-1}
\end{array}\right) \cdot\left[\begin{array}{lll}
Y_{+} & \ldots & T_{+}^{\ell-1} Y_{+}
\end{array}\right]
$$

and by Corollary 2.12 it follows that $P_{\gamma}^{\prime} \cdot P_{\gamma}^{\prime}=P_{\gamma}^{\prime}$. The inclusion

$$
\operatorname{ker}\left[\begin{array}{lll}
Y_{+} & \cdots & T_{+}^{\ell-1} Y_{+}
\end{array}\right] \subset \operatorname{ker} P_{\gamma}^{\prime}
$$

is obvious. Conversely, if $P_{\gamma}^{\prime} c=0$ then $\left[\begin{array}{lll}Y_{+} & \ldots & T_{+}^{\ell-1} Y_{+}\end{array}\right] \cdot P_{\gamma}^{\prime} c=0$, and using (10) and Corollary 2.12 we get $\left[\begin{array}{llll}Y_{+} & \cdots & T_{+}^{\ell-1} Y_{+}\end{array}\right] c=0$. Hence

$$
\operatorname{ker} P_{\gamma}^{\prime} \subset \operatorname{ker}\left[\begin{array}{lll}
Y_{+} & \cdots & T_{+}^{\ell-1} Y_{+}
\end{array}\right]
$$

so equality holds. 
Our aim now is to show the connection between the Calderón projector $P_{\gamma}$ for a matrix polynomial and the Riesz projector for an operator. (See the third corollary below.)

Theorem 2.14. Let $\gamma$ and $\Gamma$ be simple, closed contours not intersecting $\operatorname{sp}(L)$ such that $\gamma$ is contained in the interior of $\Gamma$. Let $(X, T, Y)$ be a $\Gamma$-spectral triple of $L(\lambda)$ and let

$$
Q_{\gamma}=\frac{1}{2 \pi i} \int_{\gamma}(I \lambda-T)^{-1} d \lambda
$$

the Riesz spectral projector. Let $\mathfrak{M}^{+}=\operatorname{im} Q_{\gamma}$, the image of $Q_{\gamma}$. Then

$$
\left(X_{+}, T_{+}, Y_{+}\right):=\left(X_{\mid \mathfrak{M}^{+}}, T_{\mid \mathfrak{M}^{+}}, Q_{\gamma} Y\right)
$$

is a $\gamma$-spectral triple of $L(\lambda)$ with base space $\mathfrak{M}^{+}$.

Proof. From Prop. 2.8, $\operatorname{sp}(T)$ consists of the eigenvalues of $L(\lambda)$ inside $\Gamma$. In particular, $\operatorname{sp}(T) \cap \gamma=\emptyset$, so that $Q_{\gamma}$ is well-defined. To prove that $\left(X_{+}, T_{+}, Y_{+}\right)$ is a $\gamma$-spectral triple of $L(\lambda)$, we verify conditions (i) to (iv) of Def. 2.1. Since $\operatorname{im} Q_{\gamma}$ is the invariant subspace of $T$ corresponding to the eigenvalues of $T$ inside $\gamma$, it follows that $\operatorname{sp}\left(T_{+}\right)$lies inside $\gamma$. This proves (i). To prove (iii) note that $\operatorname{col}\left(X_{+} T_{+}^{j}\right)_{j=0}^{\ell-1}=\operatorname{col}\left(X T^{j}\right)_{j=0 \mid \mathfrak{M}^{+}}^{\ell-1}$. Hence the injectivity of $\operatorname{col}\left(X_{+} T_{+}^{j}\right)_{j=0}^{\ell-1}$ follows from that of $\operatorname{col}\left(X T^{j}\right)_{j=0}^{\ell-1}$. The proof of (iv) is similar. Finally, observe that

$$
\begin{aligned}
X_{+} T_{+}^{j} Y_{+} & =X T^{j} Q_{\gamma} Y \\
& =X T^{j} \cdot \frac{1}{2 \pi i} \int_{\gamma}(I \lambda-T)^{-1} d \lambda \cdot Y \\
& =\frac{1}{2 \pi i} \int_{\gamma} \lambda^{j} X(I \lambda-T)^{-1} Y d \lambda \\
& =\frac{1}{2 \pi i} \int_{\gamma} \lambda^{j} L^{-1}(\lambda) d \lambda, \quad j=0,1, \ldots,
\end{aligned}
$$

where the last equality holds since $L^{-1}(\lambda)-X(I \lambda-T)^{-1} Y$ has an analytic continuation inside $\Gamma$ and, therefore, inside $\gamma$. Thus condition (ii') and hence (ii) holds.

Corollary. Suppose that the portion of $\operatorname{sp}(L)$ inside $\Gamma$ is divided into two parts by contours $\gamma^{+}$and $\gamma^{-}$. Let $\left(X_{ \pm}, T_{ \pm}, Y_{ \pm}\right)$be $\gamma^{ \pm}$-spectral triples of $L(\lambda)$, respectively. Then

$$
X=\left[\begin{array}{ll}
X_{+} & X_{-}
\end{array}\right], \quad T=\left(\begin{array}{cc}
T_{+} & \\
& T_{-}
\end{array}\right), \quad Y=\left(\begin{array}{l}
Y_{+} \\
Y_{-}
\end{array}\right)
$$

is a $\Gamma$-spectral triple of $L(\lambda)$.

Proof. If $(X, T, Y)$ is any $\Gamma$-spectral triple of $L(\lambda)$ (not necessarily of the form (11)) with base space $\mathfrak{M}$, let $Q_{\gamma^{ \pm}}=\frac{1}{2 \pi i} \int_{\gamma^{ \pm}}(I \lambda-T)^{-1}$ be the Riesz projectors. Then $\mathfrak{M}=\mathfrak{M}^{+} \oplus \mathfrak{M}^{-}$, where $\mathfrak{M}^{ \pm}=\operatorname{im} Q_{\gamma^{ \pm}}$, and in view of Theorem 2.14, it follows that relative to this decomposition the triple $(X, T, Y)$ may be represented in the form (11), where $\left(X_{ \pm}, T_{ \pm}, Y_{ \pm}\right):=\left(X_{\mid \mathfrak{M}^{ \pm}}, T_{\mid \mathfrak{M}^{ \pm}}, Q_{\gamma^{ \pm}} Y\right)$. Due to the fact that any two $\gamma^{+}$-spectral (resp. $\gamma^{-}$-spectral) triples of $L(\lambda)$ are similar, the corollary is proved.

Corollary. Suppose that the portion of $\operatorname{sp}(L)$ inside $\Gamma$ is divided into two disjoint parts by contours $\gamma^{+}$and $\gamma^{-}$. Define Calderón projectors $P_{\gamma^{+}}$and $P_{\gamma^{-}}$as in (8) relative to $\gamma^{+}$and $\gamma^{-}$, respectively. Then $P_{\gamma^{+}} P_{\gamma^{-}}=P_{\gamma^{-}} P_{\gamma^{+}}=0$. 
Proof. As in the preceding corollary, let

$$
X=\left[\begin{array}{ll}
X_{+} & X_{-}
\end{array}\right], \quad T=\left(\begin{array}{cc}
T_{+} & \\
& T_{-}
\end{array}\right), \quad Y=\left(\begin{array}{c}
Y_{+} \\
Y_{-}
\end{array}\right)
$$

be a $\Gamma$-spectral triple of $L(\lambda)$, where $\left(X_{ \pm}, T_{ \pm}, Y_{ \pm}\right)$are $\gamma^{ \pm}$-spectral triples of $L(\lambda)$, respectively. By Corollary $2.12, \operatorname{row}\left(T^{j} Y\right)_{j=0}^{\ell-1} \cdot \mathcal{Z}$ is a left inverse to $\operatorname{col}\left(X T^{j}\right)_{j=0}^{\ell-1}$, that is,

$$
\left(\begin{array}{ccc}
Y_{+} & \cdots & T_{+}^{\ell-1} Y_{+} \\
Y_{-} & \cdots & T_{-}^{\ell-1} Y_{-}
\end{array}\right) \cdot \mathcal{Z} \cdot\left(\begin{array}{cc}
X_{+} & X_{-} \\
\vdots & \vdots \\
X_{+} T_{+}^{\ell-1} & X_{-} T_{-}^{\ell-1}
\end{array}\right)=\left(\begin{array}{cc}
I_{\mathfrak{M}^{+}} & 0 \\
0 & I_{\mathfrak{M}^{-}}
\end{array}\right)
$$

where $I_{\mathfrak{M}^{ \pm}}$denote the identity operators on the base spaces, $\mathfrak{M}^{ \pm}$, of $\left(X_{ \pm}, T_{ \pm}, Y_{ \pm}\right)$, respectively. Hence

$$
\operatorname{row}\left(T_{+}^{j} Y_{+}\right)_{j=0}^{\ell-1} \cdot \mathcal{Z} \cdot \operatorname{col}\left(X_{-} T_{-}^{k}\right)_{k=0}^{\ell-1}=0
$$

and

$$
\operatorname{row}\left(T_{-}^{j} Y_{-}\right)_{j=0}^{\ell-1} \cdot \mathcal{Z} \cdot \operatorname{col}\left(X_{+} T_{+}^{k}\right)_{k=0}^{\ell-1}=0
$$

Now, by (9), we have

$$
P_{\gamma^{ \pm}}=\operatorname{col}\left(X_{ \pm} T_{ \pm}^{j}\right)_{j=0}^{\ell-1} \cdot \operatorname{row}\left(T_{ \pm}^{j} Y_{ \pm}\right)_{j=0}^{\ell-1} \cdot \mathcal{Z},
$$

and it follows from (12) that $P_{\gamma^{+}} P_{\gamma^{-}}=P_{\gamma^{-}} P_{\gamma^{+}}=0$.

Corollary. Suppose that the portion of $\operatorname{sp}(L)$ inside $\Gamma$ is divided into two disjoint parts by contours $\gamma^{+}$and $\gamma^{-}$. Let $(X, T)$ be a $\Gamma$-spectral pair of $L(\lambda)$. Then

$$
\operatorname{col}\left(X T^{j}\right)_{j=0}^{\ell-1} \cdot Q_{\gamma^{ \pm}}=P_{\gamma^{ \pm}} \cdot \operatorname{col}\left(X T^{j}\right)_{j=0}^{\ell-1},
$$

where $Q_{\gamma^{ \pm}}=\frac{1}{2 \pi i} \int_{\gamma^{ \pm}}(I \lambda-T)^{-1}$, the Riesz projectors, and $P_{\gamma^{ \pm}}$are the Calderón projectors (8) relative to $\gamma^{ \pm}$, respectively.

Proof. Let $\mathfrak{M}$ denote the base space of $(X, T)$. Then $\mathfrak{M}=\mathfrak{M}^{+} \oplus \mathfrak{M}^{-}$, where $\mathfrak{M}^{ \pm}=\operatorname{im} Q_{\gamma^{ \pm}}$, and corresponding to this decomposition of $\mathfrak{M}$ we may write

$$
X=\left[\begin{array}{ll}
X_{+} & X_{-}
\end{array}\right], \quad T=\left(\begin{array}{cc}
T_{+} & \\
& T_{-}
\end{array}\right),
$$

where $\left(X_{ \pm}, T_{ \pm}\right)$are $\gamma^{ \pm}$-spectral pairs of $L(\lambda)$, respectively. Then, since $P_{\gamma^{+}} P_{\gamma^{-}}=$ $P_{\gamma^{-}} P_{\gamma^{+}}=0$ and $\operatorname{im} P_{\gamma^{ \pm}}=\operatorname{im} \operatorname{col}\left(X_{ \pm} T_{ \pm}^{j}\right)_{j=0}^{\ell-1}$, we obtain

$$
\begin{aligned}
& \operatorname{col}\left(X T^{j}\right)_{j=0}^{\ell-1} \cdot Q_{\gamma^{+}}=\left[\operatorname{col}\left(X_{+} T_{+}^{j}\right)_{j=0}^{\ell-1} \quad 0\right] \\
& =P_{\gamma^{+}} \cdot\left[\operatorname{col}\left(X_{+} T_{+}^{j}\right)_{j=0}^{\ell-1} \quad \operatorname{col}\left(X_{-} T_{-}^{j}\right)_{j=0}^{\ell-1}\right] \\
& =P_{\gamma^{+}} \cdot \operatorname{col}\left(X T^{j}\right)_{j=0}^{\ell-1},
\end{aligned}
$$

and similarly for $Q_{\gamma^{-}}$and $P_{\gamma^{-}}$.

The following corollary will be needed in $\S \S 5-7$. The letter $\alpha$ denotes the degree of $\operatorname{det} L(\lambda)$, and $r$ is the number of zeros of $\operatorname{det} L(\lambda)$ inside $\gamma^{+}$. In the statement of the corollary, $\left(X_{ \pm}, T_{ \pm}, Y_{ \pm}\right)$are $\gamma^{ \pm}$-spectral triples of $L(\lambda)$ consisting of matrices. This means that $X_{+}$is an $r \times p$ matrix, $T_{+}$is an $r \times r$ matrix and $Y_{+}$is a $p \times r$ matrix, and similarly for $X_{-}, T_{-}, Y_{-}$with $r$ replaced by $\alpha-r$. 
Matrix polynomials such as $B(\lambda)$ below which have rectangular matrix coefficients arise from boundary operators for elliptic systems.

Corollary. Suppose that the portion of $\operatorname{sp}(L)$ inside $\Gamma$ is divided into two disjoint parts by contours $\gamma^{+}$and $\gamma^{-}$, and let $\left(X_{ \pm}, T_{ \pm}, Y_{ \pm}\right)$be $\gamma^{ \pm}$-spectral triples of $L(\lambda)$, consisting of matrices. Then for any $r \times r$ matrix, $M_{+}$, and $r \times(\alpha-r)$ matrix, $M_{-}$, there exists an $r \times p$ matrix polynomial, $B(\lambda)=\sum_{j=0}^{\ell-1} B_{j} \lambda^{j}$, such that

$$
\sum_{j=0}^{\ell-1} B_{j} X_{+} T_{+}^{j}=M_{+} \quad \text { and } \quad \sum_{j=0}^{\ell-1} B_{j} X_{-} T_{-}^{j}=M_{-},
$$

and its coefficients are given by

$$
B_{j}=M_{+} \cdot \sum_{k=0}^{\ell-j-1} T_{+}^{k} Y_{+} A_{j+k+1}+M_{-} \cdot \sum_{k=0}^{\ell-j-1} T_{-}^{k} Y_{-} A_{j+k+1} .
$$

Proof. Let $X=\left[\begin{array}{ll}X_{+} & X_{-}\end{array}\right], T=\left(\begin{array}{cc}T_{+} & \\ & T_{-}\end{array}\right)$, and $Y=\left(\begin{array}{l}Y_{+} \\ Y_{-}\end{array}\right)$. By the first corollary above, $(X, T, Y)$ is a finite spectral triple of $L(\lambda)$, that is, a $\Gamma$-spectral triple where $\Gamma$ is a contour having all of $\operatorname{sp}(L)$ in its interior. Since the equations (13) can be written in the form $\sum_{j=0}^{\ell-1} B_{j} X T^{j}=\left[\begin{array}{ll}M_{+} & M_{-}\end{array}\right]$, or

$$
\left[\begin{array}{lll}
B_{0} & \cdots & B_{\ell-1}
\end{array}\right] \cdot \operatorname{col}\left(X T^{j}\right)_{j=0}^{\ell-1}=\left[\begin{array}{ll}
M_{+} & M_{-}
\end{array}\right],
$$

then, in view of the fact that $\left[\begin{array}{lll}Y & \ldots & T^{\ell-1} Y\end{array}\right] \mathcal{Z}$ is a left inverse of $\operatorname{col}\left(X T^{j}\right)_{j=0}^{\ell-1}$, there is the solution $\left[\begin{array}{lll}B_{0} & \ldots & B_{\ell-1}\end{array}\right]=\left[\begin{array}{ll}M_{+} & M_{-}\end{array}\right] \cdot\left[\begin{array}{lll}Y & \ldots & T^{\ell-1} Y\end{array}\right] \mathcal{Z}$, that is,

$$
B_{j}=\left[\begin{array}{ll}
M_{+} & M_{-}
\end{array}\right] \sum_{k=0}^{\ell-j-1} T^{k} Y A_{j+k+1}
$$

which is equal to (14).

Remark. If $L(\lambda)$ has invertible leading coefficient, then the $B_{j}$ are unique since $\operatorname{col}\left(X T^{j}\right)_{j=0}^{\ell-1}$ is invertible. For the case when the leading coefficient is not invertible see [WRL, §4.2].

A matrix polynomial $L(\lambda)=I \lambda^{\ell}+\sum_{j=0}^{\ell-1} A_{j} \lambda^{j}$ in which the leading coefficient is the $p \times p$ identity matrix, $I$, is said to be monic. In this case, the degree of $\operatorname{det} L(\lambda)$ is $\alpha=p \ell$, so $\operatorname{det} L(\lambda)$ has $p \ell$ zeros. If $(X, T)$ is a finite spectral pair for $L(\lambda)$, then $\operatorname{col}\left(X T^{j}\right)_{j=0}^{\ell-1}$ is not just injective, but invertible since it is a square matrix.

Definition 2.15. Let $L(\lambda)$ be a monic $p \times p$ matrix polynomial of degree $\ell$. A pair of matrices $(X, T)$, where $X$ is $p \times p \ell$ and $T$ is $p \ell \times p \ell$, is called a standard pair of $L(\lambda)$ if the following conditions are satisfied:

(i) $\operatorname{col}\left(X T^{i}\right)_{i=0}^{\ell-1}$ is invertible;

(ii) $\sum_{j=0}^{\ell-1} A_{j} X T^{j}+X T^{\ell}=0$.

A standard pair of $L(\lambda)$ is simply a finite spectral pair. It is important, however, to have a new terminology here in order to single out the monic case. Strictly speaking, a standard pair refers only to a pair of matrices, not operators. For the proof of the following theorem, see [GLR] or [WRL]. 
Theorem 2.16. Let $L(\lambda)$ be a monic $p \times p$ matrix polynomial, and let $(X, T, Y)$ be a triple of matrices, where $X$ is $p \times p \ell, T$ is $p \ell \times p \ell$ and $Y$ is $p \ell \times p$. Then the following are equivalent:

(a) $(X, T, Y)$ is a finite spectral triple of $L(\lambda)$;

(b) $L^{-1}(\lambda)=X(I \lambda-T)^{-1} Y$;

(c) $(X, T)$ is a standard pair for $L(\lambda)$ and

$$
Y=\left(\operatorname{col}\left(X T^{i}\right)_{i=0}^{\ell-1}\right)^{-1} \cdot\left(\begin{array}{c}
0 \\
\vdots \\
0 \\
I
\end{array}\right)
$$

We call $(X, T, Y)$ a standard triple for $L(\lambda)$ if any (hence all) of these properties hold.

Remark. By Corollary 2.12 we have

$$
\left(\operatorname{col}\left(X T^{i}\right)_{i=0}^{\ell-1}\right)^{-1}=\operatorname{row}\left(T^{j} Y\right)_{j=0}^{\ell-1} \cdot \mathcal{Z},
$$

where $\mathcal{Z}$ is the matrix defined by (5) (here $A_{\ell}=I$ ).

Analogous to Def. 2.15, there is the definition of left standard pair. A pair of matrices $(T, Y)$, where $Y$ is $p \ell \times p$ and $T$ is $p \ell \times p \ell$, is called a left standard pair of $L(\lambda)$ if

(i) $\operatorname{row}\left(T^{j} Y\right)_{j=0}^{\ell-1}$ is invertible;

(ii) $\sum_{j=0}^{\ell-1} T^{j} Y A_{j}+T^{\ell} Y=0$.

It is not hard to show that $(T, Y)$ is a left standard pair for $L(\lambda)$ if and only there exists a $p \times p \ell$ matrix $X$ such that $(X, T, Y)$ is a standard triple for $L(\lambda)$. Moreover, if $(X, T, Y)$ is a, standard triple for $L(\lambda)$, then

$$
X=\left[\begin{array}{llll}
0 & \ldots & 0 & I
\end{array}\right] \cdot\left(\operatorname{row}\left(T^{j} Y\right)_{j=0}^{\ell-1}\right)^{-1} .
$$

\section{Alternative versions of the L-COndition}

As mentioned earlier, we use the matrix theory of $\S 2$ to reformulate the Lcondition for elliptic boundary problems in various equivalent forms.

Let $\mathcal{A}$ be an elliptic operator with DN numbers $s_{1}, \ldots, s_{p}, t_{1}, \ldots, t_{p}$. Associated with the DN principal part of $\mathcal{A}$ on the boundary $\partial \Omega$, there is the $p \times p$ matrix polynomial

$$
L_{y, \xi^{\prime}}(\lambda):=\pi \mathcal{A}\left(y,\left(\xi^{\prime}, \lambda\right)\right)=\sum_{j=0}^{\ell} A_{j}\left(y, \xi^{\prime}\right) \lambda^{j},
$$

$y \in \partial \Omega, 0 \neq \xi^{\prime} \in \mathrm{T}_{y}^{*}(\partial \Omega)$, where $\ell \leq \max \left(s_{i}+t_{j}\right)$. We suppose that $\mathcal{A}$ is properly elliptic, so that $\operatorname{det} L_{y, \xi^{\prime}}(\lambda)$ has $r$ roots in the upper half-plane $\operatorname{Im} \lambda>0$ and $r$ in the lower half-plane.

Remark. Recall that $\left(\xi^{\prime}, \lambda\right)$ is the short notation for $\xi^{\prime}+\lambda \cdot n(y)$. Also, the use of the letter $\ell$ is distinct from that in Theorem 1.7. 
Let $\mathcal{B}$ be a boundary operator of the type considered in $\S 1$ with DN numbers $m_{1}, \ldots, m_{r}, t_{1}, \ldots, t_{p}$. We can write it in the form

$$
\mathcal{B}(y, D)=\sum_{\kappa=0}^{\mu} \mathcal{B}_{\kappa}\left(y, D^{\prime}\right) D_{n}^{\kappa},
$$

where the coefficient-operators, $\mathcal{B}_{\kappa}=\left[b_{k j}^{\kappa}\right]$, are $r \times p$ matrices of classical p.d.o.'s on $\partial \Omega$. Note that the order of $b_{k j}^{\kappa}$ is $m_{k}+t_{j}-\kappa$. The integer $\mu \geq 0$ is the transversal order of the boundary operator and is the maximum of the orders of the normal derivatives that occur in the entries of $\mathcal{B}$. Associated with the DN principal part, $\pi \mathcal{B}$, there is the $r \times p$ matrix polynomial

$$
B_{y, \xi^{\prime}}(\lambda):=\pi \mathcal{B}\left(y,\left(\xi^{\prime}, \lambda\right)\right)=\sum_{j=0}^{\mu} B_{j}\left(y, \xi^{\prime}\right) \lambda^{j},
$$

$y \in \partial \Omega, 0 \neq \xi^{\prime} \in \mathrm{T}_{y}^{*}(\partial \Omega)$, where $B_{j}=\pi \mathcal{B}_{j}$ is the principal symbol of the $j$ th coefficient-operator, $\mathcal{B}_{j}$.

Remark. To simplify the notation, we often suppress the variables $y, \xi^{\prime}$ and write just $L(\lambda)$ rather than $L_{y, \xi^{\prime}}(\lambda)$. The same is done for $B(\lambda)$.

The goal of this section is to reformulate the L-condition for elliptic boundary value problems in several equivalent versions. We start with an abstract, quite general formulation, and use the matrix notation of $\S 2$.

Let $L(\lambda)=\sum_{j=0}^{\ell} A_{j} \lambda^{j}$ be a $p \times p$ matrix polynomial. Suppose that $\operatorname{det} L(\lambda) \neq 0$ for $\lambda \in \mathbb{R}$, and let $\gamma^{+}$be a simple, closed contour in the upper half-plane $\operatorname{Im} \lambda>0$ that contains all the eigenvalues of $L(\lambda)$ there. By $\S 2$ there exists a $\gamma^{+}$-spectral triple $\left(X_{+}, T_{+}, Y_{+}\right)$for $L(\lambda)$, and the base space of the $\gamma^{+}$-spectral pair $\left(X_{+}, T_{+}\right)$ has dimension $r$, where $r$ is the number of roots of $\operatorname{det} L(\lambda)=0$ inside $\gamma^{+}$. We let $\mathfrak{M}^{+}=\mathfrak{M}_{L\left(i^{-1} d / d t\right)}^{+}$denote the subspace of $C^{\infty}\left(\mathbb{R}, \mathbb{C}^{p}\right)$ consisting of the solutions of $L\left(i^{-1} \frac{d}{d t}\right) u=0$ such that $u(t) \rightarrow 0$ as $t \rightarrow+\infty$. Recall that $\operatorname{dim} \mathfrak{M}^{+}=r$, and by Corollary 2.10 every $u \in \mathfrak{M}^{+}$admits a representation of the form

$$
u(t)=X_{+} e^{i t T_{+}} c,
$$

for a unique $c \in \mathbb{C}^{r}$.

Remark. The matrix polynomial $L\left(\frac{1}{i} \lambda\right)$ has the spectral pair $\left(X_{+}, i T_{+}\right)$with respect to the eigenvalues in the half-plane $\operatorname{Re} \lambda<0$.

The (right) Calderón projector is

$$
P_{+}=P_{\gamma^{+}}=\frac{1}{2 \pi i} \int_{\gamma^{+}}\left(\begin{array}{c}
I \\
\vdots \\
\lambda^{\ell-1} I
\end{array}\right) L^{-1}(\lambda)\left[L_{1}(\lambda) \quad \ldots \quad L_{\ell}(\lambda)\right] d \lambda,
$$

where $L_{j}(\lambda)=A_{j}+A_{j+1} \lambda+\cdots+A_{\ell} \lambda^{\ell-j}, j=0, \ldots, \ell$, and we know that the image of $P_{+}$is equal to the set of initial conditions $\operatorname{col}\left(\left(\frac{1}{i} \frac{d}{d t}\right)^{j} u(0)\right)_{j=0}^{\ell-1}$.

Theorem 3.1. Let $B(\lambda)=\sum_{j=0}^{\mu} B_{j} \lambda^{j}$ be an $r \times p$ matrix polynomial of degree $\mu$. The following statements are equivalent:

(a) For any $y \in \mathbb{C}^{r}$, there is a unique $u \in \mathfrak{M}^{+}$such that

$$
B\left(\frac{1}{i} \frac{d}{d t}\right) u_{\mid t=0}=y
$$


(b) If $\left(X_{+}, T_{+}\right)$is a $\gamma$-spectral pair for $L(\lambda)$, where $X_{+}$is a $p \times r$ matrix and $T_{+}$ is an $r \times r$ matrix, then the $r \times r$ matrix $\Delta_{B}^{+}=\sum_{j=0}^{\mu} B_{j} X_{+} T_{+}^{j}$ is invertible, i.e.

$$
\operatorname{det} \Delta_{B}^{+}=\operatorname{det}\left(\sum_{j=0}^{\mu} B_{j} X_{+} T_{+}^{j}\right) \neq 0 .
$$

Proof. As we remarked above, any $u \in \mathfrak{M}^{+}$can be represented in the form $u(t)=$ $X_{+} e^{i t T_{+}} c$ for a unique $c \in \mathbb{C}^{r}$. The equivalence of (a) and (b) is clear since

$$
B\left(\frac{1}{i} \frac{d}{d t}\right) u_{\mid t=0}=\left(\sum_{j=0}^{\mu} B_{j} X_{+} T_{+}^{j}\right) \cdot c .
$$

Corollary. Further, if $\mu \leq \ell-1$ and we define the $r \times p \ell$ matrix

$$
\tilde{B}=\left[\begin{array}{llllll}
B_{0} & \ldots & B_{\mu} & 0 & \ldots & 0
\end{array}\right],
$$

then (a) and (b) are also equivalent to: $\left(b^{\prime}\right)$ The rank of $\tilde{B} \cdot P_{+}$is equal to $r$.

Proof. Let $\left(X_{+}, T_{+}, Y_{+}\right)$be a matrix $\gamma$-spectral triple for $L(\lambda)$. Then $X_{+}$is a $p \times r$ matrix, $T_{+}$is $r \times r$ and $Y_{+}$is $r \times p$. Consider the $r \times p \ell$ matrix $\tilde{B} \cdot P_{+}$as an operator $\mathbb{C}^{p \ell} \rightarrow \mathbb{C}^{r}$ and the $r \times r$ matrix $\sum_{j=0}^{\mu} B_{j} X_{+} T_{+}^{j}$ as an operator $\mathbb{C}^{r} \rightarrow \mathbb{C}^{r}$. Since

$$
P_{+}=\left(\begin{array}{c}
X_{+} \\
\vdots \\
X_{+} T_{+}^{\ell-1}
\end{array}\right) \cdot\left[\begin{array}{lll}
Y_{+} & \ldots & T_{+}^{\ell-1} Y_{+}
\end{array}\right] \cdot \mathcal{Z},
$$

where $\mathcal{Z}$ is defined by $(2-5)$, it is clear that the image of $\tilde{B} P_{+}$is contained in that of $\sum_{j=0}^{\mu} B_{j} X_{+} T_{+}^{j}$. Conversely, if

$$
y=\sum_{j=0}^{\mu} B_{j} X_{+} T_{+}^{j} c
$$

where $c \in \mathbb{C}^{r}$, then $y=\tilde{B} \operatorname{col}\left(X_{+} T_{+}^{j}\right)_{j=0}^{\ell-1} c$. Since the image of $P_{+}$is equal to that of $\operatorname{col}\left(X_{+} T_{+}^{j}\right)_{j=0}^{\ell-1}$, we have $\operatorname{col}\left(X_{+} T_{+}^{j}\right)_{j=0}^{\ell-1} c=P_{+} c^{\prime}$ for some $c^{\prime} \in \mathbb{C}^{p \ell}$, whence $y=\tilde{B} P_{+} c^{\prime}$. This means that $\tilde{B} P_{+}$and $\sum_{j=0}^{\mu} B_{j} X_{+} T_{+}^{j}$ have the same image, and therefore the same rank. This proves the equivalence of $(b)$ and $\left(b^{\prime}\right)$.

In the following theorem, $\tilde{L}(\lambda)$ denotes the cofactor matrix of $L(\lambda)$, i.e. the matrix polynomial such that $L(\lambda) \tilde{L}(\lambda)=\tilde{L}(\lambda) L(\lambda)=\operatorname{det} L(\lambda) \cdot I$. Also we let $P_{+}^{\prime}$ be the left Calderón projector (Theorem 2.13),

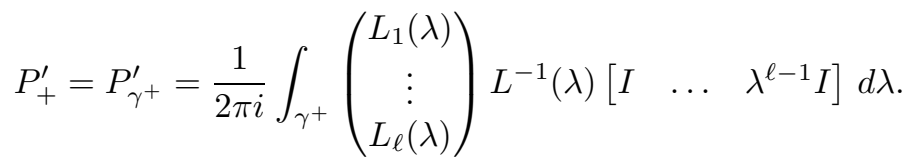

Theorem 3.2. The conditions (a) and (b) of Theorem 3.1 are also equivalent to:

(c) The $r \times p \ell$ matrix (called the Lopatinskii matrix)

$$
G=\frac{1}{2 \pi i} \int_{\gamma^{+}} B(\lambda) L^{-1}(\lambda)\left[\begin{array}{lll}
I & \ldots & \lambda^{\ell-1} I
\end{array}\right] d \lambda
$$

has rank $r$. 
(d) There exists a $p \ell \times r$ matrix $S$ such that $G S=I_{r}$ and $S G=P_{+}^{\prime}$, where $P_{+}^{\prime}$ is the left Calderón projector.

(e) The rows of the $r \times p$ matrix polynomial $B(\lambda) \cdot \tilde{L}(\lambda)$ are linearly independent modulo $\rho^{+}(\lambda)$, where we have factored the scalar polynomial $\operatorname{det} L(\lambda)$ as $\rho^{-}(\lambda) \cdot \rho^{+}(\lambda), \rho^{+}$containing all the roots of $\operatorname{det} L(\lambda)=0$ inside $\gamma^{+}$, and $\rho^{-}$ all the roots outside $\gamma^{+}$.

Proof. As a preliminary remark, note that by linear algebra the $r \times p \ell$ matrix $G$ has rank $r$ if and only if the corresponding linear map $G: \mathbb{C}^{p \ell} \rightarrow \mathbb{C}^{r}$ is surjective.

(b) $\Leftrightarrow(\mathrm{c})$. Let $\left(X_{+}, T_{+}, Y_{+}\right)$be a matrix $\gamma^{+}$-spectral triple for $L(\lambda)$. By the formula (ii') in Prop. 2.2, the Lopatinskii matrix is equal to

$$
G=\left(\sum_{j=0}^{\mu} B_{j} X_{+} T_{+}^{j}\right) \cdot\left[\begin{array}{lll}
Y_{+} & \ldots & T_{+}^{\ell-1} Y_{+}
\end{array}\right],
$$

and we know that $\left[\begin{array}{lll}Y_{+} & \ldots & T_{+}^{\ell-1} Y_{+}\end{array}\right]$has rank $r$ (see Def. 2.1(iv)). Hence $G$ has rank $r$ if and only if the $r \times r$ matrix $\Delta_{B}^{+}=\sum_{j=0}^{\mu} B_{j} X_{+} T_{+}^{j}$ is invertible.

(b) $\Leftrightarrow(\mathrm{d})$. The left Calderón projector is equal to

$$
P_{+}^{\prime}=\mathcal{Z} \cdot \operatorname{col}\left(X_{+} T_{+}^{j}\right)_{j=0}^{\ell-1} \cdot\left[\begin{array}{lll}
Y_{+} & \ldots & T_{+}^{\ell-1} Y_{+}
\end{array}\right] .
$$

If (b) holds we let

$$
S=\mathcal{Z} \cdot \operatorname{col}\left(X_{+} T_{+}^{j}\right)_{j=0}^{\ell-1} \cdot\left(\sum_{j=0}^{\mu} B_{j} X_{+} T_{+}\right)^{-1} ;
$$

then $S G=P_{+}^{\prime}$, and by Corollary 2.12 we have $G S=I_{r}$. Conversely, if (d) holds then the equation $G S=I_{r}$ implies that $G$ has rank $r$. In view of (5) this implies that the matrix $\Delta_{B}^{+}=\sum_{j=0}^{\mu} B_{j} X_{+} T_{+}^{j}$ has rank $r$; hence it is invertible since it is a square $r \times r$ matrix.

(c) $\Rightarrow\left(\right.$ e). Note that $\tilde{L}(\lambda)=\operatorname{det} L(\lambda) \cdot L^{-1}(\lambda)$; as remarked above, $\tilde{L}(\lambda)$ is the cofactor matrix for $L(\lambda)$, so it is a polynomial. If $x \in \mathbb{C}^{r}$ is such that

$$
x B(\lambda) \tilde{L}(\lambda)=\rho^{+}(\lambda) \cdot M(\lambda)
$$

for some $1 \times p$ matrix polynomial $M(\lambda)$, then dividing (8) by $\operatorname{det} L(\lambda)$ gives

$$
x B(\lambda) L^{-1}(\lambda)=\rho^{-}(\lambda)^{-1} \cdot M(\lambda),
$$

and the right-hand side is holomorphic inside $\gamma$, whence

$$
x \int_{\gamma^{+}} B(\lambda) L^{-1}(\lambda)\left[\begin{array}{lll}
I & \ldots & \lambda^{\ell-1} I
\end{array}\right] d \lambda=0 .
$$

Thus (c) implies that $x=0$.

(e) $\Rightarrow$ (c). Conversely, let $x \in \mathbb{C}^{r}$ be such that (9) holds. In view of (5) we see that $x \cdot \sum_{j=0}^{\mu} B_{j} X_{+} T_{+}^{j}=0$. Multiplying both sides of this equation by $T_{+}^{k} Y_{+}$for $k=0,1, \ldots$, and making use of the formula (ii') in Prop. 2.2, we obtain

$$
x \int_{\gamma^{+}} B(\lambda) L^{-1}(\lambda) \lambda^{k} d \lambda=0
$$

for all $k=0,1, \ldots$. Hence $x B(\lambda) L^{-1}(\lambda)$ has an analytic continuation inside $\gamma$ as a matrix function of $\lambda$. Since $\tilde{L}(\lambda)=\operatorname{det} L(\lambda) \cdot L^{-1}(\lambda)$, this means that $x B(\lambda) \cdot \tilde{L}(\lambda)$ vanishes at the roots of $\operatorname{det} L(\lambda)=0$ inside $\gamma^{+}$, i.e. vanishes at the 
roots of $\rho^{+}(\lambda)=0$. Hence (8) holds for some $1 \times p$ matrix polynomial $M(\lambda)$, and then (e) implies that $x=0$. Thus $G$ is surjective, i.e. has rank $r$.

Remark. The matrix $S$ is uniquely determined by the conditions in (d). The first equation implies that $G$ is surjective, and if $S$ and $S_{1}$ satisfy $S G=P_{+}^{\prime}$ and $S_{1} G=$ $P_{+}^{\prime}$ then $\left(S-S_{1}\right) G=0$, whence $S-S_{1}=0$.

Remark. From the proof of (c) $\Leftrightarrow$ (e) it is clear that condition (e) can be formulated in another way: The only $x \in \mathbb{C}^{r}$ such that $x B(\lambda) \cdot L^{-1}(\lambda)$ has an analytic continuation inside $\gamma^{+}$is $x=0$.

There is a natural correspondence between the conditions (a) and (c), i.e. depending only on $L(\lambda)$, not on the choice of spectral triple. First of all, note that (5), (6) and Corollary 2.12 imply that $G \cdot P_{+}^{\prime}=G$. Also, for the map $\phi: \mathbb{C}^{p \ell} \rightarrow \mathfrak{M}^{+}$ defined by

$$
c \mapsto \phi c(t)=\frac{1}{2 \pi i} \int_{\gamma^{+}} e^{i t \lambda} L^{-1}(\lambda) \cdot \sum_{j=0}^{\ell-1} c_{j+1} \lambda^{j} d \lambda,
$$

we have $\left.B\left(\frac{1}{i} \frac{d}{d t}\right)\right|_{t=0} \circ \phi=G$, and, in view of Prop. 2.5, $\phi$ is surjective. Further, since

$$
\phi c=X_{+} e^{i t T_{+}}\left[\begin{array}{lll}
Y_{+} & \cdots & T_{+}^{\ell-1} Y_{+}
\end{array}\right] \cdot c,
$$

it follows that $\operatorname{ker} \phi=\operatorname{ker}\left[\begin{array}{lll}Y_{+} & \ldots & T_{+}^{\ell-1} Y_{+}\end{array}\right]$, which is equal to ker $P_{+}^{\prime}$ by Theorem 2.13. Thus we have the diagram

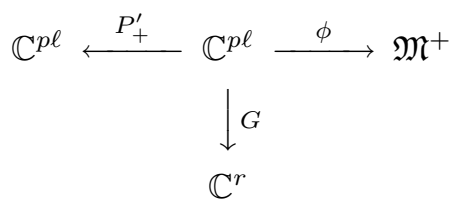

where the fact that $P_{+}^{\prime}$ and $\phi$ have the same kernel means that we can identify im $P_{+}^{\prime}$ and $\mathfrak{M}^{+}$along the top row of the diagram. With this identification, the map $\left.G\right|_{\operatorname{im} P_{+}^{\prime}}: \operatorname{im} P_{+}^{\prime} \rightarrow \mathbb{C}^{r}$ is identified with the map $\left.B\left(\frac{1}{i} \frac{d}{d t}\right)\right|_{t=0}: \mathfrak{M}^{+} \rightarrow \mathbb{C}^{r}$. Thus, condition (a) holds if and only if

$$
\left.G\right|_{\mathrm{im} P_{+}^{\prime}}: \operatorname{im} P_{+}^{\prime} \longrightarrow \mathbb{C}^{r} \quad \text { is bijective. }
$$

Now we apply the matrix theory to the L-condition for elliptic systems $(\mathcal{A}, \mathcal{B})$. For the sake of emphasis we gather all the equivalent conditions together in one long theorem.

From now on we usually omit mention of the contour $\gamma^{+}$, i.e. we write $\int_{+}$instead of $\int_{\gamma^{+}}$, because the value of the contour integral depends only on the residues in the upper half-plane $\operatorname{Im} \lambda>0$, not on the particular contour.

In the following theorem, no assumptions are made concerning the transversal order, $\mu$, of the boundary operator ( $\mu \geq \ell$ is permitted).

Theorem 3.3. Suppose that the operator $\mathcal{A}(x, D)$ is properly elliptic, let $\mathcal{B}(y, D)$ be a boundary operator as in (1), and fix $y \in \partial \Omega, 0 \neq \xi^{\prime} \in \mathrm{T}_{y}^{*}(\partial \Omega)$. The following statements are equivalent:

(i) The initial value problem

$$
\pi \mathcal{A}\left(y,\left(\xi^{\prime}, \frac{1}{i} \frac{d}{d t}\right)\right) u(t)=0, \quad t>0
$$




$$
\left.\pi \mathcal{B}\left(y,\left(\xi^{\prime}, \frac{1}{i} \frac{d}{d t}\right)\right) u(t)\right|_{t=0}=g,
$$

has for every choice of $g \in \mathbb{C}^{r}$ a unique solution $u \in \mathfrak{M}_{L\left(\frac{1}{i} \frac{d}{d t}\right)}^{+}$. As usual, $\mathfrak{M}_{L\left(\frac{1}{i} \frac{d}{d t}\right)}^{+}$is the space of solutions of (10) such that $u(t) \rightarrow 0$ as $t \rightarrow \infty$ (i.e. corresponding to the eigenvalues of $L(\lambda)$ with a positive imaginary part).

(ii) The $r \times p \ell$ matrix $G=G_{y, \xi^{\prime}}$ defined by

$$
G=\int_{+} \pi \mathcal{B}\left(y,\left(\xi^{\prime}, \lambda\right)\right) \pi \mathcal{A}\left(y,\left(\xi^{\prime}, \lambda\right)\right)^{-1}\left[\begin{array}{lll}
I & \ldots & \lambda^{\ell-1} I
\end{array}\right] d \lambda
$$

has rank $r$, where $\int_{+}$denotes the integral along a simple, closed contour $\gamma^{+}$ in the upper half-plane containing all roots of $\operatorname{det} L_{y, \xi^{\prime}}(\lambda)=0$ with a positive imaginary part.

(iii) If $\left(X_{+}\left(y, \xi^{\prime}\right), T_{+}\left(y, \xi^{\prime}\right), Y_{+}\left(y, \xi^{\prime}\right)\right)$ is a $\gamma^{+}$-spectral triple for $L_{y, \xi^{\prime}}(\lambda)$, where $X_{+}\left(y, \xi^{\prime}\right)$ is a $p \times r$ matrix, $T_{+}\left(y, \xi^{\prime}\right)$ an $r \times r$ matrix and $Y_{+}\left(y, \xi^{\prime}\right)$ an $r \times p$ matrix (existence by Theorem 2.6), then

$$
\operatorname{det} \Delta_{\mathcal{B}}^{+}\left(y, \xi^{\prime}\right)=\operatorname{det}\left(\sum_{j=0}^{\mu} B_{j}\left(y, \xi^{\prime}\right) X_{+}\left(y, \xi^{\prime}\right) T_{+}^{j}\left(y, \xi^{\prime}\right)\right) \neq 0 .
$$

(iv) There is a unique $p \ell \times r$ matrix $S=S_{y, \xi^{\prime}}$ such that $G S=I_{r}$ and $S G=P_{+}^{\prime}$, where $P_{+}^{\prime}=P_{\gamma^{+}}^{\prime}$ is the left Calderón projector for $L_{y, \xi^{\prime}}(\lambda)$.

(v) Let us factor the scalar polynomial, $\operatorname{det} L_{y, \xi^{\prime}}(\lambda)$, as $\rho^{-}(\lambda) \cdot \rho^{+}(\lambda)$, where $\rho^{+}$ contains all the roots above the real axis, and $\rho^{-}$contains all roots below the real axis. If $\tilde{L}(\lambda)$ denotes the matrix polynomial

$$
\tilde{L}(\lambda)=\operatorname{det} L_{y, \xi^{\prime}}(\lambda) \cdot L_{y, \xi^{\prime}}^{-1}(\lambda),
$$

then the rows of $B_{y, \xi^{\prime}}(\lambda) \cdot \tilde{L}(\lambda)$ are linearly independent modulo $\rho^{+}(\lambda)$.

The first condition is of course equivalent to the L-condition stated in Def. 1.5. Condition (ii) is known as the Lopatinskii condition; the matrix (11) is the Lopatinskii matrix, and was introduced by Lopatinskii in his paper [Lo]. Fedosov used condition (iv) in a series of papers, beginning with [Fe], where he developed an index formula for elliptic boundary value problems. The last condition (v) is called the "covering" or "complementing condition", and was introduced and used by Agmon, Douglis and Nirenberg in their fundamental paper [ADN].

An example illustrating the use of the $\Delta$-condition is given at the end of this section, but first we must define the Dirichlet problem for an elliptic operator.

The Dirichlet problem. Let $\mathcal{A}$ be a $p \times p$ properly elliptic operator, and suppose that $r=p s$ for some $s$. Then we can pose the Dirichlet problem $(\mathcal{A}, \mathcal{D})$, where

$$
\mathcal{D}=\left(\begin{array}{c}
I \\
I \partial_{n} \\
\vdots \\
I \partial_{n}^{s-1}
\end{array}\right), \quad I \text { is } p \times p \text { identity, } \quad \partial_{n}=\partial / \partial n,
$$

but in general it will not satisfy the L-condition. In fact, if we let

$$
\left(X_{+}\left(y, \xi^{\prime}\right), T_{+}\left(y, \xi^{\prime}\right), Y_{+}\left(y, \xi^{\prime}\right)\right)
$$

be a $\gamma^{+}$-spectral triple for $L_{y, \xi^{\prime}}(\lambda)$, then Theorem 3.3 implies the following result: 
The Dirichlet problem, $(\mathcal{A}, \mathcal{D})$, satisfies the L-condition if and only if the $r \times r$ matrix

$$
\Delta_{\mathcal{D}}^{+}=\operatorname{col}\left(X_{+}\left(y, \xi^{\prime}\right) T_{+}^{j}\left(y, \xi^{\prime}\right)\right)_{j=0}^{s-1}
$$

is invertible for all $\left(y, \xi^{\prime}\right) \in \mathrm{T}^{*}(\partial \Omega) \backslash 0$.

If $\gamma$ is a simple, closed contour not intersecting $\operatorname{sp}(L)$, then a matrix polynomial $L(\lambda)$ is said to admit a $\gamma$-spectral right divisor $L_{1}(\lambda)$ if $L(\lambda)=L_{2}(\lambda) \cdot L_{1}(\lambda)$, where the spectra, $\operatorname{sp}\left(L_{1}\right)$ and $\operatorname{sp}\left(L_{2}\right)$, lie inside and outside of $\gamma$, respectively. It can be shown that the invertibility of (13) is equivalent to the existence of a $\gamma^{+}$-spectral monic right divisor of degree $s$ for $L_{y, \xi^{\prime}}(\lambda)$ for all $y \in \partial \Omega$ and $0 \neq \xi^{\prime} \in \mathrm{T}_{y}^{*}(\partial \Omega)$; see [GLR, Chap. 4] or [WRL, Chap. 3].

Assume now that the principal part of $\mathcal{A}$ is homogeneous of degree $\ell$ (Def. 1.3). In this case the leading coefficient of $L_{y, \xi^{\prime}}(\lambda)$ is $A_{\ell}=\pi \mathcal{A}(y, n(y))$, which is invertible. Then $\operatorname{det} L_{y, \xi^{\prime}}(\lambda)$ has degree $p \ell$. In order to pose the Dirichlet problem we need $r=p s$ for some $s$, hence $\ell=2 s$.

Theorem 3.4. Let $\mathcal{A}(x, D)$ be a properly elliptic operator and suppose that the principal part of $\mathcal{A}$ is homogeneous of degree $\ell=2 s$. The Dirichlet problem $(\mathcal{A}, \mathcal{D})$ satisfies the L-condition if and only if

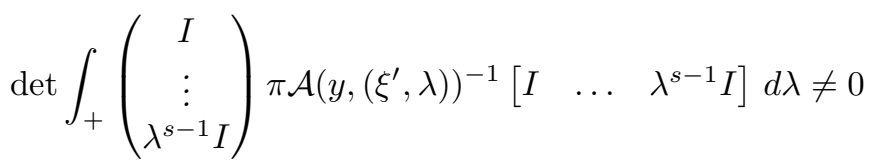

for all $y \in \partial \Omega, 0 \neq \xi^{\prime} \in \mathrm{T}_{y}^{*}(\partial \Omega)$.

Proof. Suppose there is a $\gamma^{+}$-spectral right factorization

$$
L_{y, \xi^{\prime}}(\lambda)=L_{y, \xi^{\prime}}^{-}(\lambda) L_{y, \xi^{\prime}}^{+}(\lambda)
$$

with $L_{y, \xi^{\prime}}^{+}(\lambda)$ monic of degree $s$. Then $L_{y, \xi^{\prime}}^{-}(\lambda)$ must also have degree $s$ with invertible leading coefficient, since $A_{\ell}$ is invertible and $\ell=2 s$. Due to the homogeneity of $\pi \mathcal{A}$ we have $\pi \mathcal{A}\left(y,\left(\xi^{\prime}, \lambda\right)\right)=(-1)^{\ell} \pi \mathcal{A}\left(y,\left(-\xi^{\prime},-\lambda\right)\right)$, and hence

$$
L_{y, \xi^{\prime}}(\lambda)=(-1)^{\ell} L_{y,-\xi^{\prime}}^{-}(-\lambda) L_{y,-\xi^{\prime}}^{+}(-\lambda) .
$$

This means that whenever $L_{y, \xi^{\prime}}(\lambda)$ has a monic $\gamma^{+}$-spectral right divisor of degree $s$ for all $y \in \partial \Omega, 0 \neq \xi^{\prime} \in \mathrm{T}_{y}^{*}(\partial \Omega)$, it also has a monic $\gamma^{+}$-spectral left divisor of degree $s$. As mentioned above, the existence of a monic $\gamma^{+}$-spectral right divisor is equivalent to the invertibility of the matrix (13). By duality, it is also true that the existence of a monic $\gamma^{+}$-spectral left divisor is equivalent to invertibility of the matrix

$$
\operatorname{row}\left(T_{+}^{j}\left(y, \xi^{\prime}\right) Y_{+}\left(y, \xi^{\prime}\right)\right)_{j=0}^{s-1}
$$

Now, observe that by virtue of Prop. 2.2 the matrix in (14) is equal to

$$
\left(\begin{array}{c}
X_{+} \\
\vdots \\
X_{+} T_{+}^{s-1}
\end{array}\right) \cdot\left[\begin{array}{lll}
Y_{+} & \cdots & T_{+}^{s-1} Y_{+}
\end{array}\right]
$$

which implies that (14) holds if and only if both the matrices (13), (15) are invertible. 
In checking the Lopatinskii condition we are confronted by the difficulty of deciding which $r$ of the $\ell p(\ell=2 s)$ columns of (11) are independent. The theorem above shows that for the Dirichlet problem it is only necessary to check for linear independence in the first $r$ columns. The same is true for any boundary operator once the Dirichlet problem is known to satisfy the Lopatinskii condition:

Theorem 3.5. Let the principal part of the operator $\mathcal{A}$ be homogeneous of degree $\ell=2 s$ and suppose that the Dirichlet problem $(\mathcal{A}, \mathcal{D})$ satisfies the $L$-condition, i.e. the $p s \times p s$ matrix (14) is invertible. Then the $p s \times p$ boundary operator $\mathcal{B}(y, D)$ satisfies the $L$-condition relative to $\mathcal{A}$ if and only if

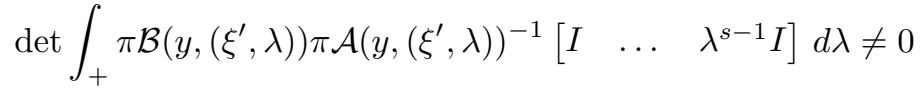

for all $y \in \partial \Omega, 0 \neq \xi^{\prime} \in \mathrm{T}_{y}^{*}(\partial \Omega)$.

Proof. Let $\left(X_{+}, T_{+}, Y_{+}\right)$be a $\gamma^{+}$-spectral triple for $L_{y, \xi^{\prime}}(\lambda)$. As mentioned above, the invertibility of (14) is equivalent to the invertibility of both factors in (16). Now, since the matrix in (17) is equal to

$$
\sum_{j=0}^{\mu} B_{j} X_{+} T_{+}^{j} \cdot\left[\begin{array}{lll}
Y_{+} & \cdots & T_{+}^{s-1} Y_{+}
\end{array}\right]
$$

the invertibility of $\left[\begin{array}{lll}Y_{+} & \ldots & T_{+}^{s-1} Y_{+}\end{array}\right]$implies that (18) has non-zero determinant if and only if $\sum_{j=0}^{\mu} B_{j} X_{+} T_{+}^{j}$ has non-zero determinant. By (12) this is equivalent to the L-condition for $(\mathcal{A}, \mathcal{B})$.

For an application of Theorem 3.5, see [Ro2, Theorem 7.5]. We end this section with an example to illustrate the use of the $\Delta$-condition of Theorem 3.3.

Example. Let $\varphi(x)=\varphi_{1}(x)+i \varphi_{2}(x)$, and consider the following $2 \times 2$ operator of order $\ell=2$ :

$$
\mathcal{A}\left(x, \frac{\partial}{\partial x}\right)=A(x) \frac{\partial^{2}}{\partial x_{2}^{2}}+2 B(x) \frac{\partial^{2}}{\partial x_{2} \partial x_{1}}+C(x) \frac{\partial^{2}}{\partial x_{1}^{2}}+\text { lower-order terms, }
$$

where

$$
A(x)=\left(\begin{array}{cc}
\varphi_{1}(x) & \varphi_{2}(x)+1 \\
\varphi_{2}(x)-1 & -\varphi_{1}(x)
\end{array}\right), \quad B(x)=-I, \quad C(x)=A(x)^{T}
$$

and $\varphi_{1}, \varphi_{2}$ are real-valued functions in $C^{\infty}(\bar{\Omega})$ such that

$$
\left(\varphi_{1}(x)\right)^{2}+\left(\varphi_{2}(x)\right)^{2} \neq 1
$$

for all $x \in \bar{\Omega}$. The principal part of $\mathcal{A}$ is

$$
\begin{aligned}
\pi \mathcal{A}(x, \xi) & =A(x) \xi_{2}^{2}+2 B(x) \xi_{1} \xi_{2}+C(x) \xi_{1}^{2} \\
& =\left(\begin{array}{cc}
\varphi_{1}(x)\left(\xi_{1}^{2}+\xi_{2}^{2}\right)-2 \xi_{1} \xi_{2} & \varphi_{2}(x)\left(\xi_{1}^{2}+\xi_{2}^{2}\right)+\left(\xi_{2}^{2}-\xi_{1}^{2}\right) \\
\varphi_{2}(x)\left(\xi_{1}^{2}+\xi_{2}^{2}\right)-\left(\xi_{2}^{2}-\xi_{1}^{2}\right) & -\varphi_{1}(x)\left(\xi_{1}^{2}+\xi_{2}^{2}\right)-2 \xi_{1} \xi_{2}
\end{array}\right),
\end{aligned}
$$

where $\xi=\left(\xi_{1}, \xi_{2}\right) \in \mathbb{R}^{2}$. Then

$$
\operatorname{det} \pi \mathcal{A}(x, \xi)=\left(\xi_{1}^{2}+\xi_{2}^{2}\right)^{2}\left(1-|\varphi(x)|^{2}\right),
$$

and in view of the assumption (20) ellipticity holds, i.e. $\operatorname{det} \pi \mathcal{A}(x, \xi) \neq 0$ for all $\xi \neq 0$. Here we have $\ell=2 s$, where $s=1$, and $r=p s=2$.

Let $n(y)$ be the inward-pointing unit normal at $y \in \partial \Omega$, and $\tau(y)$ the unit tangent vector to $\partial \Omega$ such that $\tau(y), n(y)$ is a positively oriented basis of $\mathbb{R}^{2}$, i.e. 
$\tau(y)$ points in the counterclockwise direction. Writing $\tau(y)=\left(\tau_{1}(y), \tau_{2}(y)\right)$, we have $n(y)=\left(-\tau_{2}(y), \tau_{1}(y)\right)$. We will consider two boundary problems for $\mathcal{A}$ : (I) The Dirichlet boundary problem

$$
\mathcal{A} u=f(x), x \in \Omega, \quad \mathcal{B} u=g(y), y \in \partial \Omega,
$$

where $\mathcal{B} u=u \mid \partial \Omega$; and (II) a Neumann-type boundary problem

$$
\mathcal{A} u=f(x), x \in \Omega, \quad \mathcal{C} u=g(y), y \in \partial \Omega
$$

where $\mathcal{C} u=C_{2}(y) \partial / \partial x_{2}+C_{1}(y) \partial / \partial x_{1}$ and $C_{2}=\tau_{1} A-\tau_{2} B, C_{1}=\tau_{1} B-\tau_{2} C$. The goal is to find the conditions on the function $\varphi$ that ensure the boundary problem (21) or (22) satisfies the L-condition. By homogeneity of $\pi \mathcal{A}$ it suffices to verify the L-condition when $\left|\xi^{\prime}\right|=1$, i.e. $\left(y, \xi^{\prime}\right) \in \mathrm{ST}^{*}(\partial \Omega)$; see $\S 5$, equation (11). We identify $\mathrm{ST}^{*}(\partial \Omega)$ with the unit tangent bundle $\mathrm{ST}(\partial \Omega)$. If $\left(y, \xi^{\prime}\right) \in \mathrm{ST}^{*}(\partial \Omega)$, then $\left|\xi^{\prime}\right|=1$, so $\xi^{\prime}= \pm \tau(y)$, and $\operatorname{ST}^{*}(\partial \Omega)$ is the disjoint union of two copies of $\partial \Omega$. The $2 \times 2$ matrix polynomial associated with $\mathcal{A}$ on the boundary $\partial \Omega$ is $L_{y, \xi^{\prime}}(\lambda)=\pi \mathcal{A}\left(y, \xi^{\prime}+\lambda \cdot n(y)\right)$, where $\xi^{\prime}= \pm \tau(y)$. Now let $\tilde{L}_{y}(\lambda)=L_{y, \tau(y)}(\lambda)$, that is,

$$
\tilde{L}_{y}(\lambda)=\pi \mathcal{A}(y, \tau(y)+\lambda \cdot n(y))=\tilde{A}(y) \lambda^{2}+2 \tilde{B}(y) \lambda+\tilde{C}(y)
$$

Remark. When $\xi^{\prime}=-\tau(y)$ we have $L_{y,-\tau(y)}(\lambda)=\tilde{L}_{y}(-\lambda)$. The operator $\mathcal{A}$ and boundary operators $\mathcal{B}, \mathcal{C}$ have real matrix coefficients; thus by virtue of complex conjugation it suffices to verify the L-condition when $\xi^{\prime}=\tau(y)$. See Remark 1.1 in [Ro2].

To investigate the L-condition for the boundary problem (21) or (22), observe that the matrix polynomials associated with the boundary operators are

$$
\tilde{B}_{y}(\lambda)=\pi \mathcal{B}(y, \tau(y)+\lambda \cdot n(y))=I
$$

and

$$
\tilde{C}_{y}(\lambda)=\pi \mathcal{C}(y, \tau(y)+\lambda \cdot n(y))=\tilde{A}(y) \lambda+\tilde{B}(y),
$$

respectively, where $I$ denotes the $2 \times 2$ identity matrix and the $2 \times 2$ matrix functions $\tilde{A}(y), \tilde{B}(y)$ are defined by the equation (23). In view of Theorem 3.4 we must find the conditions on $\varphi$ ensuring that

$$
\operatorname{det} \int_{+} \tilde{L}_{y}(\lambda)^{-1} d \lambda \neq 0
$$

for the Dirichlet problem (21), and in view of Theorem 3.3(ii)

$$
\operatorname{rank} \int_{+} \tilde{C}_{y}(\lambda) \tilde{L}_{y}(\lambda)^{-1}\left[\begin{array}{ll}
I & \lambda I
\end{array}\right] d \lambda=2
$$

for the Neumann problem (22). It is cumbersome to compute these integrals directly, so let us use the $\Delta$-condition of Theorem 3.3(iii). To do so requires that we have a $\gamma^{+}$-spectral pair of $\tilde{L}_{y}(\lambda)$. To lessen the computational burden, consider first the related matrix polynomial:

$$
\begin{aligned}
L_{x}(\lambda) & =\pi \mathcal{A}(x,(1, \lambda)) \\
& =A(x) \lambda^{2}+2 B(x) \lambda+C(x) \\
& =\left(\begin{array}{cc}
\varphi_{1}(x)\left(\lambda^{2}+1\right)-2 \lambda & \varphi_{2}(x)\left(\lambda^{2}+1\right)+\left(\lambda^{2}-1\right) \\
\varphi_{2}(x)\left(\lambda^{2}+1\right)-\left(\lambda^{2}-1\right) & -\varphi_{1}(x)\left(\lambda^{2}+1\right)-2 \lambda
\end{array}\right)
\end{aligned}
$$


and $\operatorname{det} L_{x}(\lambda)=\left(1+\lambda^{2}\right)^{2}\left(1-|\varphi(x)|^{2}\right)$ for all $\lambda \in \mathbb{R}$, where $\varphi(x)=\varphi_{1}(x)+i \varphi_{2}(x)$. Note that $L_{x}(\lambda)$ has two eigenvalues $\lambda= \pm i$, both of multiplicity two. It is not hard to verify that

$$
X_{+}(x)=\left(\begin{array}{cc}
1 & -i \varphi_{2}(x) \\
-i & i \varphi_{1}(x)
\end{array}\right), \quad T_{+}(x)=\left(\begin{array}{cc}
i & 1 \\
0 & i
\end{array}\right)
$$

is a $\gamma^{+}$-spectral pair of $L_{x}(\lambda)$ for all $x \in \bar{\Omega}$. (The matrix $T_{+}$is a Jordan block and the columns of $X_{+}$are a Jordan chain, corresponding to the eigenvalue $\lambda=i$. See [WRL, Example 3.17].)

Note that $L_{x}(\lambda)$ is defined in terms of the basis $(1,0),(0,1)$ for $\mathbb{R}^{2}$, whereas the matrix polynomial $\tilde{L}_{y}(\lambda)=\pi \mathcal{A}(y, \tau(y)+\lambda n(y))$ is defined in terms of the basis $\tau(y), n(y)$. Since $\tau(y)=\left(\tau_{1}(y), \tau_{2}(y)\right)$ and $n(y)=\left(-\tau_{2}(y), \tau_{1}(y)\right)$, we have

$$
\tilde{L}_{y}(\lambda)=\left(\tau_{1}(y)-\lambda \tau_{2}(y)\right)^{2} \cdot L_{y}\left(\varphi^{-1}(\lambda)\right),
$$

where $\varphi^{-1}(\lambda)=\left(\tau_{2}+\lambda \tau_{1}\right) /\left(\tau_{1}-\lambda \tau_{2}\right)$. Since $\varphi$ maps the upper half-plane $\operatorname{Im} \lambda>0$ to itself, then by [WRL, Theorem 2.21] the pair

$$
\begin{aligned}
\tilde{X}_{+}(y) & =X_{+}(y), \\
\tilde{T}_{+}(y) & =\left(\tau_{1}(y) T_{+}(y)-\tau_{2}(y) I\right)\left(\tau_{2}(y) T_{+}(y)+\tau_{1}(y) I\right)^{-1}
\end{aligned}
$$

is a $\gamma^{+}$-spectral pair of $\tilde{L}_{y}(\lambda)$. For the Dirichlet problem with $s=1$ we have $\Delta_{\mathcal{B}}^{+}(y, \tau(y))=\tilde{X}_{+}(y)$. Then $\operatorname{det} \Delta_{\mathcal{B}}^{+}(y, \tau(y))=i \overline{\varphi(y)}$, and it follows that the Dirichlet problem (21) satisfies the L-condition if and only if (20) holds and

$$
\varphi(y) \neq 0 \text { for all } y \in \partial \Omega \text {. }
$$

For the Neumann problem we have

$$
\Delta_{\mathcal{C}}^{+}(y, \tau(y))=\tilde{A}(y) \tilde{X}_{+}(y) \tilde{T}_{+}(y)+\tilde{B}(y) \tilde{X}_{+}(y),
$$

and, after some computation, one obtains that

$$
\Delta_{\mathcal{C}}^{+}(y, \tau(y))=\left(\begin{array}{cc}
\overline{i \overline{\varphi(y)}} & -i \\
-\overline{\varphi(y)} & |\varphi(y)|^{2}-1
\end{array}\right)
$$

whence $\operatorname{det} \Delta_{\mathcal{C}}^{+}(y, \tau(y))=i \overline{\varphi(y)}\left(|\varphi(y)|^{2}-2\right)$. Thus the Neumann problem (22) satisfies the L-condition if and only if (20) holds and

$$
\varphi(y) \neq 0, \quad|\varphi(y)| \neq \sqrt{2} \text { for all } y \in \partial \Omega
$$

\section{Matrix polynomials DePEnding on Parameters}

Let $M$ be a $C^{\infty}$ manifold, and let

$$
L_{x}(\lambda)=\sum_{j=0}^{\ell} A_{j}(x) \lambda^{j}, \quad x \in M,
$$

be a family of $p \times p$ matrix polynomials of degree $\leq \ell$ with matrix coefficients which are $C^{\infty}$ functions of $x \in M$ and such that $\operatorname{det} L_{x}(\lambda) \neq 0$ for all real $\lambda$. We also assume that the number, $r$, of zeros of $\operatorname{det} L_{x}(\lambda)$ in the upper half-plane $\operatorname{Im} \lambda>0$ is locally constant on $M$, multiplicities counted. This is equivalent to the following assumption. For any $x_{0} \in M$ and simple, closed contour $\gamma^{+}$containing the zeros of $\operatorname{det} L_{x_{0}}(\lambda)$ in $\operatorname{Im} \lambda>0$ :

$\left(^{*}\right)$ There is an open set $U \ni x_{0}$ such that $\gamma^{+}$contains the zeros of $\operatorname{det} L_{x}(\lambda)$ in $\operatorname{Im} \lambda>0$ for all $x \in U$. 
We do not assume that the degree of $\operatorname{det} L_{x}(\lambda)$ is constant, although for matrix polynomials that arise from an elliptic operator this will always be true, of course.

For each $x \in M$, there is the vector space $\mathfrak{M}_{x}^{+}$of solutions of $L_{x}\left(\frac{1}{i} \frac{d}{d t}\right) u=0$ such that $u(t) \rightarrow 0$ as $t \rightarrow+\infty$. Let $\gamma_{x}^{+}$be a simple, closed contour containing the eigenvalues of $L_{x}(\lambda)$ in the upper half-plane $\operatorname{Im} \lambda>0$. As in the proof of Theorem 2.6, $L_{x}(\lambda)$ has the $\gamma_{x}^{+}$-spectral triple $\left(\left.X_{+}\right|_{x},\left.T_{+}\right|_{x},\left.Y_{+}\right|_{x}\right)$, where

$$
\begin{aligned}
\left.X_{+}\right|_{x}: \mathfrak{M}_{x}^{+} \rightarrow \mathbb{C}^{p} \quad \text { maps } \quad u \mapsto u(0), \\
\left.T_{+}\right|_{x}: \mathfrak{M}_{x}^{+} \rightarrow \mathfrak{M}_{x}^{+} \quad \text { maps } \quad u \mapsto \frac{1}{i} \frac{d u}{d t}, \\
\left.Y_{+}\right|_{x}: \mathbb{C}^{p} \rightarrow \mathfrak{M}_{x}^{+} \quad \text { maps } \quad c \mapsto \frac{1}{2 \pi i} \int_{+} e^{i t \lambda} L_{x}^{-1}(\lambda) c d \lambda .
\end{aligned}
$$

Lemma 4.1. Let $L_{x}(\lambda)$ have the properties stated above. The family of vector spaces $\mathfrak{M}^{+}=\left\{\mathfrak{M}_{x}^{+}\right\}$has a unique vector bundle structure over $M$ such that the maps $X_{+}: \mathfrak{M}^{+} \rightarrow \mathbb{C}^{p}, T_{+}: \mathfrak{M}^{+} \rightarrow \mathfrak{M}^{+}$and $Y_{+}: \mathbb{C}^{p} \rightarrow \mathfrak{M}^{+}$defined on each fiber by (2) are vector bundle homomorphisms.

Proof. The Calderón projector for $L_{x}(\lambda)$ with respect to the eigenvalues in the upper half-plane is given by

$$
P_{x}^{+}=\frac{1}{2 \pi i} \int_{\gamma_{x}^{+}}\left(\begin{array}{c}
I \\
\vdots \\
\lambda^{\ell-1} I
\end{array}\right) L_{x}^{-1}(\lambda)\left[\begin{array}{lll}
I & \ldots & \lambda^{\ell-1} I
\end{array}\right] \mathcal{Z}_{x} d \lambda,
$$

where $\gamma_{x}^{+}$contains the eigenvalues of $L_{x}(\lambda)$ in the upper half-plane $\operatorname{Im} \lambda>0$. Let $x_{0} \in M$. By assumption, see $(*)$, there exists a neighbourhood $U$ of $x_{0}$ such that the contour $\gamma_{x}^{+}$in (3) can be replaced by $\gamma_{x_{0}}^{+}$for all $x \in U$. Since the coefficients of $L_{x}(\lambda)$ depend smoothly on $x$ it is clear that the matrix $P_{x}^{+}$depends smoothly on $x$. Hence $P_{x}^{+}$is a projection operator for the trivial bundle $M \times \mathbb{C}^{p \ell}$. Hence the family of vector spaces $\operatorname{im} P_{+}=\left\{\operatorname{im} P_{x}^{+}\right\}$is a vector bundle over $M$ (see [WRL, $\S 5.7])$.

We have a map $\varphi: \mathfrak{M}^{+} \rightarrow \operatorname{im} P_{+}$defined by the Cauchy data on each fiber,

$$
u \in \mathfrak{M}_{x}^{+} \longmapsto \mathcal{U}=\operatorname{col}\left(\left(\frac{1}{i} \frac{d}{d t}\right)^{j} u(0)\right)_{j=0}^{\ell-1} \in \operatorname{im} P_{x}^{+} \subset \mathbb{C}^{p \ell},
$$

which we know is a linear isomorphism on each fiber (Theorem 2.9). We give $\mathfrak{M}^{+}$the vector bundle structure that makes $\varphi$ into a vector bundle isomorphism. To prove that $X_{+}, T_{+}$and $Y_{+}$are smooth we just have to show that $X_{+} \circ \varphi^{-1}$, $\varphi \circ T_{+} \circ \varphi^{-1}$ and $\varphi \circ Y_{+}$are smooth. Recall that for any matrix polynomial, $L(\lambda)$, every $u \in \mathfrak{M}^{+}$has a representation of the form

$$
u(t)=\frac{1}{2 \pi i} \int_{+} e^{i t \lambda} L^{-1}(\lambda)\left[\begin{array}{lll}
I & \ldots & \lambda^{\ell-1} I
\end{array}\right] \mathcal{Z} \cdot \mathcal{U} d \lambda,
$$

where $\mathcal{U}$ is the Cauchy data of $u$ (see (2-3) and recall that $\int_{+}$is the short notation for $\left.\int_{\gamma^{+}}\right)$. It follows that $X_{+} \circ \varphi^{-1}=\left.\mathcal{X}\right|_{\operatorname{im} P_{+}}, \varphi \circ T_{+} \circ \varphi^{-1}=\left.\mathcal{T}\right|_{\text {im } P_{+}}$and 


$$
\begin{aligned}
\varphi \circ Y_{+} & =\mathcal{Y}, \text { where } \\
\mathcal{X} & =\frac{1}{2 \pi i} \int_{+} L^{-1}(\lambda)\left[\begin{array}{llll}
I & \ldots & \lambda^{\ell-1} I
\end{array}\right] \mathcal{Z} d \lambda, \quad p \times p \ell \text { matrix, } \\
\mathcal{T} & =\frac{1}{2 \pi i} \int_{+} \operatorname{col}\left(\lambda^{j} I\right)_{j=0}^{\ell-1} \cdot \lambda L^{-1}(\lambda)\left[\begin{array}{lll}
I & \ldots & \lambda^{\ell-1} I
\end{array}\right] \mathcal{Z} d \lambda, \quad p \ell \times p \ell \text { matrix }, \\
\mathcal{Y} & =\frac{1}{2 \pi i} \int_{+} \operatorname{col}\left(\lambda^{j} I\right)_{j=0}^{\ell-1} \cdot L^{-1}(\lambda) d \lambda, \quad p \ell \times p \text { matrix. }
\end{aligned}
$$

Since $\mathcal{X}, \mathcal{T}$ and $\mathcal{Y}$ are smooth matrix functions on $M$, and im $P_{+}$is a subbundle of $M \times \mathbb{C}^{p \ell}$, it follows that $X_{+} \circ \varphi^{-1} \in \mathcal{L}\left(\operatorname{im} P_{+}, \mathbb{C}^{p}\right), \varphi \circ T_{+} \circ \varphi^{-1} \in \mathcal{L}\left(\operatorname{im} P_{+}\right)$and $\varphi \circ Y_{+} \in \mathcal{L}\left(\mathbb{C}^{p}, \operatorname{im} P_{+}\right)$are smooth.

The definitions we had previously in $\S 2$ for admissible triples of operators carry over to families of such admissible triples. A triple of vector bundle homomorphisms $(X, T, Y)$ is called an admissible triple over $M$ if $X \in \mathcal{L}\left(E, \mathbb{C}^{p}\right), T \in \mathcal{L}(E)$ and $Y \in \mathcal{L}\left(\mathbb{C}^{p}, E\right)$, where $E$ is a vector bundle over $M$. As before, $E$ is called the base space of $(X, T, Y)$. Two admissible triples over $M,(X, T, Y)$ and $\left(X^{\prime}, T^{\prime}, Y^{\prime}\right)$, with base spaces $E$ and $E^{\prime}$, respectively, are called similar if there exists a vector bundle isomorphism $\varphi \in \mathcal{L}\left(E^{\prime}, E\right)$ such that $X^{\prime}=X \varphi, T^{\prime}=\varphi^{-1} T \varphi$ and $Y^{\prime}=\varphi^{-1} Y$.

Theorem 4.2. Let $L_{x}(\lambda)=\sum_{j=0}^{\ell} A_{j}(x) \lambda^{j}$ be a family of matrix polynomials with the properties stated above. For each $x \in M$, let $\gamma_{x}^{+}$be a simple, closed contour containing the eigenvalues of $L_{x}(\lambda)$ in the upper half-plane $\operatorname{Im} \lambda>0$. Then there exists an admissible triple $\left(X_{+}, T_{+}, Y_{+}\right)$over $M$ such that $\left(X_{+}(x), T_{+}(x), Y_{+}(x)\right)$ is a $\gamma_{x}^{+}$-spectral triple of $L_{x}(\lambda)$ for all $x \in M$. Any two such admissible triples are similar.

Note. We call the triple $\left(X_{+}, T_{+}, Y_{+}\right)$a spectral triple with respect to the upper half-plane for the family of matrix polynomials $L(\lambda)=L_{x}(\lambda), x \in M$.

Proof. The existence of a spectral triple $\left(X_{+}, T_{+}, Y_{+}\right)$has been proved in Lemma 4.1 (the base space is $\left.E=\mathfrak{M}^{+}\right)$. Now let $\left(X_{+}^{\prime}, T_{+}^{\prime}, Y_{+}^{\prime}\right)$ be another spectral triple for the family $L(\lambda)$, i.e. an admissible triple over $M$, with base space $E^{\prime}$, such that $\left(X_{+}^{\prime}(x), T_{+}^{\prime}(x), Y_{+}^{\prime}(x)\right)$ is a $\gamma_{x}^{+}$-spectral triple of $L_{x}(\lambda)$ for all $x \in M$. By [WRL, Prop. 2.12], it follows that $X_{+}^{\prime}(x)=X_{+}(x) \cdot \mathcal{M}_{x}, T_{+}^{\prime}(x)=\mathcal{M}_{x}^{-1} \cdot T_{+}(x) \cdot \mathcal{M}_{x}$ and $Y_{+}^{\prime}(x)=\mathcal{M}_{x}^{-1} \cdot Y_{+}(x)$ for all $x \in M$, where

$$
\mathcal{M}_{x}=\operatorname{row}\left(T_{+}^{j}(x) Y_{+}(x)\right)_{j=0}^{\ell-1} \cdot \mathcal{Z}_{x} \cdot \operatorname{col}\left(X_{+}^{\prime}(x) T_{+}^{\prime}(x)\right)_{j=0}^{\ell-1} .
$$

Obviously $\mathcal{M} \in \mathcal{L}\left(E^{\prime}, E\right)$ and is a (smooth) isomorphism. Hence $\left(X_{+}, T_{+}, Y_{+}\right)$and $\left(X_{+}^{\prime}, T_{+}^{\prime}, Y_{+}^{\prime}\right)$ are similar.

Remark. If $\left(X_{+}, T_{+}, Y_{+}\right)$is any admissible triple over $M$ satisfying the conditions of Theorem 4.2, then its base space $E$ is necessarily isomorphic to $\mathfrak{M}^{+}$, due to the isomorphism $E \rightarrow \mathfrak{M}^{+}$given by $E_{x} \ni v \mapsto X_{+}(x) e^{i t T_{+}(x)} v \in \mathfrak{M}_{x}^{+}$.

Corollary 4.3. There exist vector bundle isomorphisms $\mathfrak{M}^{+} \simeq \operatorname{im} P_{+}$and $\mathfrak{M}^{+} \simeq$ $\operatorname{im} P_{+}^{\prime}$, where $P_{+}$and $P_{+}^{\prime}$ are the right and left Calderón projectors with respect to the eigenvalues in the upper half-plane.

Proof. The isomorphism $\mathfrak{M}^{+} \rightarrow \operatorname{im} P_{+}$is given by the Cauchy data

$$
u \mapsto \operatorname{col}\left(u^{(j)}(0)\right)_{j=0}^{\ell-1}
$$


on each fiber. As for the second isomorphism, consider the map $\phi: \mathbb{C}^{p \ell} \rightarrow \mathfrak{M}^{+}$ defined by

$$
c \longmapsto \phi c(t)=\frac{1}{2 \pi i} \int_{+} e^{i t \lambda} L^{-1}(\lambda) \cdot \sum_{j=0}^{\ell-1} c_{j+1} \lambda^{j} d \lambda .
$$

Let $\left(X_{+}, T_{+}, Y_{+}\right)$be a spectral triple with respect to the upper half-plane for the family $L(\lambda)$. Since $\phi c=X_{+} e^{i t T_{+}}$, it follows due to injectivity of $\operatorname{col}\left(X_{+} T_{+}^{j}\right)_{j=0}^{\ell-1}$ that $\operatorname{ker} \phi=\operatorname{ker}\left[\begin{array}{lll}Y_{+} & \ldots & T_{+}^{\ell-1} Y_{+}\end{array}\right]=\operatorname{ker} P_{+}^{\prime}$ (for the second equality, see Theorem 2.13). Since $P_{+}^{\prime}$ is a smooth projector, then $\operatorname{ker} P_{+}^{\prime}$ is a vector bundle over $M$. By surjectivity of $\phi$, we have an isomorphism

$$
\mathbb{C}^{p \ell} / \operatorname{ker} \phi \simeq \mathfrak{M}^{+} .
$$

But also $\mathbb{C}^{p \ell} / \operatorname{ker} \phi=\mathbb{C}^{p \ell} / \operatorname{ker} P_{+}^{\prime} \simeq \operatorname{im} P_{+}^{\prime}$, so there is a natural isomorphism $\mathfrak{M}^{+} \simeq \operatorname{im} P_{+}^{\prime}$.

In $\S \S 5-7$ it will be clear that the triple $\left(X_{+}, T_{+}, Y_{+}\right)$is a key step in the construction of boundary operators satisfying the L-condition for a given elliptic operator; this in turn is crucial for the proof of the index formula for elliptic systems in the plane. (See [Ro2].)

In fact we would like to have spectral triples $\left(X_{+}, T_{+}, Y_{+}\right)$which are $C^{\infty}$ matrix functions on $M$. (Precisely, $X_{+}(x)$ is a $p \times r$ matrix, $T_{+}(x)$ is an $r \times r$ matrix and $Y_{+}(x)$ is an $r \times p$ matrix, with entries depending smoothly on $x \in M$.) One necessary condition for the existence of such a triple $\left(X_{+}, T_{+}, Y_{+}\right)$is obvious: $\mathfrak{M}^{+}$ must be trivial, since there is the isomorphism from $M \times \mathbb{C}^{r}$ to $\mathfrak{M}^{+}$defined by

$$
(x, v) \in\{x\} \times \mathbb{C}^{r} \longmapsto X_{+}(x) e^{i t T_{+}(x)} v \in \mathfrak{M}_{x}^{+} .
$$

The triviality of $\mathfrak{M}^{+}$is also sufficient, as we show in the next proposition.

Note that the condition that the number of eigenvalues of $L_{x}(\lambda)$ in $\operatorname{Im} \lambda>0$ be locally constant in $x \in M$ just means that the number of them is constant on each component of $M$.

Theorem 4.4. As usual, suppose that $\operatorname{det} L_{x}(\lambda) \neq 0$ for all real $\lambda$, and let the number, $r$, of roots of $\operatorname{det} L_{x}(\lambda)=0$ in the upper half-plane be independent of $x \in M$. Then there exists a spectral triple $\left(X_{+}, T_{+}, Y_{+}\right)$with respect to the upper half-plane for the family $L(\lambda)=L_{x}(\lambda)$ consisting of $C^{\infty}$ matrix functions on $M$ if and only if the vector bundle $\mathfrak{M}^{+}$is trivial.

Proof. First, we claim that the base space $E$ of any spectral triple $\left(X_{+}, T_{+}, Y_{+}\right)$is isomorphic to $\mathfrak{M}^{+}$. Indeed, there is a map $\Phi: E \rightarrow \mathfrak{M}^{+}$defined on each fiber by

$$
v \in E_{x} \longmapsto X_{+}(x) e^{i t T_{+}(x)} v \in \mathfrak{M}_{x}^{+},
$$

which is a linear isomorphism on each fiber (Corollary 2.10). Since $\Phi$ is smooth it follows that $\Phi$ is a vector bundle isomorphism.

Suppose now that $\mathfrak{M}^{+}$is trivial. Then $E$ is also trivial, i.e. there exist $C^{\infty}$ sections $v_{1}, \ldots, v_{r}$ of $E$ such that, for all $x \in M$, the vectors $v_{1}(x), \ldots, v_{r}(x)$ form a basis of $E_{x}$. Relative to this basis, the vector bundle homomorphisms $X_{+}, T_{+}$ and $Y_{+}$are $C^{\infty}$ matrix functions. 
The following is an example of a family of matrix polynomials $L_{x}(\lambda)$ for which the vector bundle $\mathfrak{M}^{+}$is not trivial. For $x=\left(x_{1}, x_{2}, x_{3}\right) \in S^{2}$, the unit sphere in $\mathbb{R}^{3}$, let

$$
L_{x}(\lambda)=\left(\begin{array}{ccccc}
\lambda^{2}+1 & & & x_{1} \\
& \lambda^{2}+1 & & x_{2} \\
& & \lambda^{2}+1 & x_{3} \\
x_{1} & x_{2} & x_{3} & 0
\end{array}\right)
$$

Since det $L_{x}(\lambda)=-\left(\lambda^{2}+1\right)^{2}$, the family of matrix polynomials $L_{x}(\lambda)$ satisfies the hypotheses stated at the beginning of this section, with $r=2$. It is not hard to show that $\mathfrak{M}^{+}$is isomorphic to the tangent bundle $\mathrm{T}\left(S^{2}\right)$, and is therefore not trivial.

\section{Homogeneity PROPERTIES OF SPECTRAL TRIPLES}

Let $\mathcal{A}(x, D)$ be an elliptic differential operator in $\bar{\Omega}$ with the DN numbers $s_{1}, \ldots, s_{p}, t_{1}, \ldots, t_{p}$. The coefficients of the matrix polynomial

$$
L(\lambda)=\pi \mathcal{A}\left(y,\left(\xi^{\prime}, \lambda\right)\right)=\sum_{j=0}^{\ell} A_{j}\left(y, \xi^{\prime}\right) \lambda^{j}
$$

depend smoothly on the parameters $\left(y, \xi^{\prime}\right) \in \mathrm{T}^{*}(\partial \Omega) \backslash 0$ and have certain homogeneity properties in $\xi^{\prime}$ which follow from the homogeneity properties of $\pi \mathcal{A}$.

We consider first the case where $\mathcal{A}$ is homogeneously elliptic, that is, $s_{1}=\cdots=$ $s_{p}=\ell$ and $t_{1}=\cdots=t_{p}=0$ (see Def. 1.3), because the notation is simpler then and some of the results take on a more complete form. $L(\lambda)$ has degree $\ell$ with invertible leading coefficient, $A_{\ell}=\pi \mathcal{A}(y, n(y))$, and $\operatorname{det} L(\lambda)$ has degree $\alpha=p \ell$. A finite spectral pair $(X, T)$ for $L(\lambda)$ is therefore a standard pair (Def. $2.15)$, that is, $\operatorname{col}\left(X T^{j}\right)_{j=0}^{\ell-1}$ is invertible. The homogeneity of $\pi \mathcal{A}$ implies $L_{y, c \xi^{\prime}}(\lambda)=$ $c^{\ell} L_{y, \xi^{\prime}}\left(c^{-1} \lambda\right)$, whence

$$
A_{j}\left(y, c \xi^{\prime}\right)=c^{\ell-j} \cdot A_{j}\left(y, \xi^{\prime}\right), \quad j=0, \ldots, \ell, \quad \forall c \in \mathbb{C} .
$$

Let $\mathrm{ST}^{*}(\partial \Omega)$ denote the unit cotangent bundle to $\partial \Omega$ consisting of $\left(y, \xi^{\prime}\right) \in \mathrm{T}^{*}(\partial \Omega)$ with $\left|\xi^{\prime}\right|=1$, and let $s: \mathrm{T}^{*}(\partial \Omega) \backslash 0 \rightarrow \mathrm{ST}^{*}(\partial \Omega)$ be the map defined by $\left(y, \xi^{\prime}\right) \mapsto$ $\left(y, \xi^{\prime} /\left|\xi^{\prime}\right|\right)$.

Lemma 5.1. There exists a spectral triple $\left(X_{+}, T_{+}, Y_{+}\right)$with respect to the upper half-plane for the family $L(\lambda)=\pi \mathcal{A}\left(y,\left(\xi^{\prime}, \lambda\right)\right),\left(y, \xi^{\prime}\right) \in \mathrm{T}^{*}(\partial \Omega) \backslash 0$, such that for any $c>0$

$$
\begin{aligned}
& X_{+}\left(y, c \xi^{\prime}\right)=X_{+}\left(y, \xi^{\prime}\right), \\
& T_{+}\left(y, c \xi^{\prime}\right)=c \cdot T_{+}\left(y, \xi^{\prime}\right), \\
& Y_{+}\left(y, c \xi^{\prime}\right)=c^{1-\ell} \cdot Y_{+}\left(y, \xi^{\prime}\right),
\end{aligned}
$$

where $X_{+} \in \mathcal{L}\left(s^{-1} E, \mathbb{C}^{p}\right), T_{+} \in \mathcal{L}\left(s^{-1} E\right), Y_{+} \in \mathcal{L}\left(\mathbb{C}^{p}, s^{-1} E\right)$, and $s^{-1} E$ is the pullback to $\mathrm{T}^{*}(\partial \Omega) \backslash 0$ of a vector bundle $E$ over $\mathrm{ST}^{*}(\partial \Omega)$. (E is necessarily isomorphic to $\mathfrak{M}^{+}$.)

Proof. By virtue of Theorem 4.2 there exists a $\gamma^{+}$-spectral triple $\left(X_{+}, T_{+}, Y_{+}\right)$for $L(\lambda)$ with $X_{+} \in \mathcal{L}\left(E, \mathbb{C}^{p}\right), T_{+} \in \mathcal{L}(E), Y_{+} \in \mathcal{L}\left(\mathbb{C}^{p}, E\right)$ for some vector bundle $E$ 
over $\mathrm{ST}^{*}(\partial \Omega)$. Then we define

$$
\begin{aligned}
X_{+}\left(y, \xi^{\prime}\right) & =X_{+}\left(y, \xi^{\prime} /\left|\xi^{\prime}\right|\right), \\
T_{+}\left(y, \xi^{\prime}\right) & =\left|\xi^{\prime}\right| T_{+}\left(y, \xi^{\prime} /\left|\xi^{\prime}\right|\right), \\
Y_{+}\left(y, \xi^{\prime}\right) & =\left|\xi^{\prime}\right|^{1-\ell} Y_{+}\left(y, \xi^{\prime} /\left|\xi^{\prime}\right|\right) .
\end{aligned}
$$

The triple $\left(X_{+}, T_{+}, Y_{+}\right)$satisfies the homogeneity properties listed above, and it remains to show that it satisfies properties (i), (ii'), (iii) and (iv) of Def. 2.1 for all $\left(y, \xi^{\prime}\right) \in \mathrm{T}^{*}(\partial \Omega) \backslash 0$, given that it satisfies them on $\mathrm{ST}^{*}(\partial \Omega)$. Condition (i) obviously holds. As for condition (ii'), we have

$$
X_{+}\left(y, c \xi^{\prime}\right) T_{+}^{j}\left(y, c \xi^{\prime}\right) Y_{+}\left(y, c \xi^{\prime}\right)=\frac{1}{2 \pi i} \int_{+} \lambda^{j} L_{y, c \xi^{\prime}}^{-1}(\lambda) d \lambda,
$$

for $\left|\xi^{\prime}\right|=1, c>0, j=0,1, \ldots$, because it holds when $c=1$ and due to (1), (2) it then holds for any $c>0$ (by making the substitution $\lambda \rightarrow c^{-1} \lambda$ ). Finally, conditions (iii), (iv) hold since for $c>0$

$$
\operatorname{col}\left(X_{+}\left(y, c \xi^{\prime}\right) T_{+}^{j}\left(y, c \xi^{\prime}\right)\right)_{j=0}^{\ell-1}=F(c) \cdot \operatorname{col}\left(X_{+}\left(y, \xi^{\prime}\right) T_{+}^{j}\left(y, \xi^{\prime}\right)\right)_{j=0}^{\ell-1}
$$

and

$$
\operatorname{row}\left(T_{+}^{j}\left(y, c \xi^{\prime}\right) Y_{+}\left(y, c \xi^{\prime}\right)\right)_{j=0}^{\ell-1}=\operatorname{row}\left(T_{+}^{j}\left(y, \xi^{\prime}\right) Y_{+}\left(y, \xi^{\prime}\right)\right)_{j=0}^{\ell-1} \cdot E(c),
$$

where $F(c)=\operatorname{diag}\left(c^{j} I\right)_{j=0}^{\ell-1}$ and $E(c)=c \cdot \operatorname{diag}\left(c^{j} I\right)_{j=0}^{\ell-1}$ are $p \ell \times p \ell$ diagonal matrices which are invertible.

For the matrix $\mathcal{Z}$ defined in (2-5), the homogeneity properties (1) imply

$$
\mathcal{Z}\left(y, c \xi^{\prime}\right)=E^{-1}(c) \mathcal{Z}\left(y, \xi^{\prime}\right) F^{-1}(c),
$$

where $E(c)$ and $F(c)$ are defined as above. Hence for the right and left Calderón projectors $P_{+}$and $P_{+}^{\prime},(3-4),(3-6)$ and (2) give

$$
\begin{aligned}
& P_{+}\left(y, c \xi^{\prime}\right)=F(c) P_{+}\left(y, \xi^{\prime}\right) F^{-1}(c), \\
& P_{+}^{\prime}\left(y, c \xi^{\prime}\right)=E(c) P_{+}^{\prime}\left(y, \xi^{\prime}\right) E^{-1}(c) .
\end{aligned}
$$

Remark. If we write $P_{+}=\left[P_{k i}\right]$, where $P_{k i}$ are $p \times p$ blocks $(i, k=0, \ldots, \ell-1)$, then (6) implies $P_{k i}\left(y, c \xi^{\prime}\right)=c^{k-i} P_{k i}\left(y, \xi^{\prime}\right)$, whence $P_{k i}$ is the principal symbol of a pseudo-differential operator on $\partial \Omega$ of order $k-i$. Similarly for $P_{+}^{\prime}$.

From now on it is always assumed that the $\gamma^{+}$-spectral triple of $L(\lambda)$ is chosen with the properties (2). By repeating the proof of Lemma 5.1 we see that the triple

$$
\begin{aligned}
& \left(X_{-}\left(y, \xi^{\prime}\right), T_{-}\left(y, \xi^{\prime}\right), Y_{-}\left(y, \xi^{\prime}\right)\right) \\
& \quad:=\left(X_{+}\left(y,-\xi^{\prime}\right),-T_{+}\left(y,-\xi^{\prime}\right),(-1)^{1-\ell} Y_{+}\left(y,-\xi^{\prime}\right)\right)
\end{aligned}
$$

is a $\gamma^{-}$-spectral triple of $L_{y, \xi^{\prime}}(\lambda)$, and has the (positive) homogeneity properties (2). The Calderón projectors $P_{+}\left(y,-\xi^{\prime}\right)$ and $P_{-}\left(y, \xi^{\prime}\right)$ are similar (with similarity matrix $F(-1)$ ), and the same is true for the left Calderón projectors.

Let $\mathcal{B}$ be a boundary operator and consider the associated $r \times p$ matrix polynomial

$$
B(\lambda)=\pi \mathcal{B}\left(y,\left(\xi^{\prime}, \lambda\right)\right)=\sum_{j=0}^{\mu} B_{j}\left(y, \xi^{\prime}\right) \lambda^{j} .
$$


The $k$ th row of $\pi \mathcal{B}$ is homogeneous of degree $m_{k}$ in the variables $\xi=\left(\xi^{\prime}, \lambda\right)$, whence the $k$ th row of the coefficient $B_{j}$ is homogeneous of degree $m_{k}-j$. In other words

$$
B_{j}\left(y, c \xi^{\prime}\right)=M(c) \cdot B_{j}\left(y, \xi^{\prime}\right) c^{-j}, \quad c>0,
$$

for $j=0, \ldots, \mu$, where $M(c)$ is the $r \times r$ diagonal matrix $\left[c^{m_{k}} \delta_{k i}\right]$. Now let

$$
\Delta_{\mathcal{B}}^{+}\left(y, \xi^{\prime}\right)=\sum_{j=0}^{\mu} B_{j}\left(y, \xi^{\prime}\right) X_{+} T_{+}^{j}
$$

(we omit the argument $\left(y, \xi^{\prime}\right)$ in $X_{+}$and $T_{+}$to simplify the notation). By Theorem $3.3\left(\right.$ iii), $\mathcal{B}$ satisfies the L-condition relative to $\mathcal{A}$ if and only if $\Delta_{\mathcal{B}}^{+}\left(y, \xi^{\prime}\right)$ is invertible for all $\left(y, \xi^{\prime}\right) \in \mathrm{T}^{*}(\partial \Omega) \backslash 0$. By virtue of $(2)$ and $(9)$

$$
\Delta_{\mathcal{B}}^{+}\left(y, c \xi^{\prime}\right)=M(c) \cdot \Delta_{\mathcal{B}}^{+}\left(y, \xi^{\prime}\right), \quad c>0
$$

so it suffices to verify invertibility of $\Delta_{\mathcal{B}}^{+}\left(y, \xi^{\prime}\right)$ when $\left(y, \xi^{\prime}\right) \in \mathrm{ST}^{*}(\partial \Omega)$.

Remark. If the rows of $\pi \mathcal{B}(y, \xi)$ are not only positive homogeneous but also negative homogeneous in $\xi=\left(\xi^{\prime}, \lambda\right)$, then the $B_{j}$ 's satisfy the homogeneity property (9) for all $c \neq 0$. In such a case we also define

$$
\Delta_{\mathcal{B}}^{-}\left(y, \xi^{\prime}\right)=\sum_{j=0}^{\mu} B_{j}\left(y, \xi^{\prime}\right) X_{-} T_{-}^{j}
$$

and it follows from (9) with $c=-1$ and (8) that

$$
\Delta_{\mathcal{B}}^{+}\left(y,-\xi^{\prime}\right)=M(-1) \cdot \Delta_{\mathcal{B}}^{-}\left(y, \xi^{\prime}\right)
$$

The next proposition shows that if $\mu \leq \ell-1$ and $\pi \mathcal{B}_{1}, \pi \mathcal{B}_{2}$ are positive and negative homogeneous, then $\Delta_{\mathcal{B}_{1}}^{+}=\Delta_{\mathcal{B}_{2}}^{+}$if and only if $\pi \mathcal{B}_{1}=\pi \mathcal{B}_{2}$. In other words, given the pair $\left(X_{+}, T_{+}\right)$, the principal part $\pi \mathcal{B}$ of $\mathcal{B}$ is uniquely determined by $\Delta_{\mathcal{B}}^{+}$. Recall that $\mu$ is the transversal order; there is no restriction on the total order of $\mathcal{B}$ because the $m_{k}$ 's are permitted to be any real numbers.

Remark. The condition that $\pi \mathcal{B}$ be both positive and negative homogeneous holds if $\mathcal{B}$ is a differential operator.

Proposition 5.2. Let $\mathcal{B}$ be a boundary operator of transversal order $\mu \leq \ell-1$. Then we have $\pi \mathcal{B}\left(y,\left(\xi^{\prime}, \lambda\right)\right)=\sum_{j=0}^{\ell-1} B_{j}\left(y, \xi^{\prime}\right) \lambda^{j}$, where

$$
B_{j}=\Delta_{\mathcal{B}}^{+} \cdot \sum_{k=0}^{\ell-j-1} T_{+}^{k} Y_{+} A_{j+k+1}+\Delta_{\mathcal{B}}^{-} \cdot \sum_{k=0}^{\ell-j-1} T_{-}^{k} Y_{-} A_{j+k+1},
$$

$j=0, \ldots, \ell-1$ (and $B_{j}=0$ if $\left.j>\mu\right)$. Thus, if $\pi \mathcal{B}(y, \xi)$ is positive and negative homogeneous in $\xi$, it follows from $\left(11^{\prime}\right)$ that the principal part $\pi \mathcal{B}$ is uniquely determined by $\Delta_{\mathcal{B}}^{+}$.

Proof. Since $\sum_{j=0}^{\ell-1} B_{j}\left(y, \xi^{\prime}\right) X_{ \pm} T_{ \pm}^{j}=\Delta_{\mathcal{B}}^{ \pm}$, this follows immediately from the fourth corollary of Theorem 2.14, because $\operatorname{col}\left(X T^{j}\right)_{j=0}^{\ell-1}$ is invertible.

As we know from $\S 4$, the family of vector spaces $\mathfrak{M}^{+}=\left\{\mathfrak{M}_{L_{y, \xi^{\prime}}^{+}}^{+}\right\}$is an $r$ dimensional vector bundle over $\mathrm{ST}^{*}(\partial \Omega)$. A boundary operator $\mathcal{B}$ that satisfies 
the L-condition relative to $\mathcal{A}$ defines a trivialization

$$
\begin{aligned}
\mathfrak{M}^{+} & \longrightarrow \operatorname{ST}^{*}(\partial \Omega) \times \mathbb{C}^{r}, \\
u \in \mathfrak{M}_{L_{y, \xi^{\prime}}}^{+} & \longrightarrow B_{y, \xi^{\prime}}\left(\frac{1}{i} \frac{d}{d t}\right) u_{\mid t=0} .
\end{aligned}
$$

This means that there is a "topological obstruction" to the existence of boundary operators satisfying the L-condition. Conversely, if it is assumed that $\mathfrak{M}^{+}$is a trivial bundle, then there exists a boundary operator $\mathcal{B}$ satisfying the L-condition. In fact we have the following theorem.

Theorem 5.3. Suppose that $\mathfrak{M}^{+}$is a trivial bundle. Let $\Delta: \mathrm{ST}^{*}(\partial \Omega) \rightarrow \mathrm{GL}_{r}(\mathbb{C})$ be any $C^{\infty}$ matrix function (for example, $\Delta \equiv I$ ). Then for any $m_{k} \in \mathbb{R}, k=$ $1, \ldots, r$, there exists a boundary operator $\mathcal{B}$ with transversal order $\mu \leq \ell-1$ such that the kth row of the principal part $\pi \mathcal{B}$ is both positive and negative homogeneous of degree $m_{k}$ and

$$
\Delta_{\mathcal{B}}^{+}=\Delta \quad \text { on } \operatorname{ST}^{*}(\partial \Omega) .
$$

Moreover, the principal part of $\mathcal{B}$ is uniquely determined by these conditions. Since $\Delta$ is invertible, $\mathcal{B}$ satisfies the L-condition.

Proof. In Theorem 4.4 we showed that if $\mathfrak{M}^{+}$is trivial then there exists a $\gamma^{+}$spectral triple of $L(\lambda)$ that consists of $C^{\infty}$ matrix functions on $\mathrm{ST}^{*}(\partial \Omega)$, i.e. $X_{+}, T_{+}$ and $Y_{+}$are $C^{\infty}$ matrix functions on $\mathrm{ST}^{*}(\partial \Omega)$ of dimensions $p \times r, r \times r$ and $r \times p$, respectively. This triple of matrices can be extended by homogeneity so that it satisfies the properties (2). Now let $\left(X_{-}, T_{-}, Y_{-}\right)$be the $\gamma^{-}$-spectral triple of $L(\lambda)$ defined as in (8). In view of the first corollary to Theorem 2.14, the triple

$$
X=\left[\begin{array}{ll}
X_{+} & X_{-}
\end{array}\right], \quad T=\left(\begin{array}{cc}
T_{+} & \\
& T_{-}
\end{array}\right), \quad Y=\left(\begin{array}{c}
Y_{+} \\
Y_{-}
\end{array}\right)
$$

is a finite spectral triple of $L(\lambda)$.

Extend $\Delta$ to $\mathrm{T}^{*}(\partial \Omega) \backslash 0$ by the formula $\Delta\left(y, \xi^{\prime}\right)=M\left(\left|\xi^{\prime}\right|\right) \cdot \Delta\left(y, \xi^{\prime} /\left|\xi^{\prime}\right|\right)$, and then define matrix functions $\Delta_{ \pm}: \mathrm{T}^{*}(\partial \Omega) \backslash 0 \rightarrow \mathrm{GL}_{r}(\mathbb{C})$ by

$$
\Delta_{+}=\Delta \quad \text { and } \quad \Delta_{-}\left(y, \xi^{\prime}\right)=M(-1) \cdot \Delta\left(y,-\xi^{\prime}\right) .
$$

Now define $r \times p$ matrix functions $B_{j}, j=0,1, . ., \ell-1$, by the formulas (12) with $\Delta_{\mathcal{B}}^{ \pm}$replaced by $\Delta_{ \pm}$. Let $B_{j}^{+}\left(y, \xi^{\prime}\right)$ and $B_{j}^{-}\left(y, \xi^{\prime}\right)$ denote the + and - terms on the right-hand side of (12); then due to (2) and (1) it follows that for $c>0$

$$
\begin{aligned}
B_{j}^{+}\left(y, c \xi^{\prime}\right) & =\Delta_{+}\left(y, c \xi^{\prime}\right) \cdot \sum_{k=0}^{\ell-j-1} T_{+}^{k}\left(y, c \xi^{\prime}\right) Y_{+}\left(y, c \xi^{\prime}\right) A_{j+k+1}\left(y, c \xi^{\prime}\right) \\
& =M(c) \Delta_{+}\left(y, \xi^{\prime}\right) \cdot \sum_{k=0}^{\ell-j-1} c^{k} T_{+}^{k}\left(y, \xi^{\prime}\right) c Y_{+}\left(y, \xi^{\prime}\right) A_{j+k+1}\left(y, \xi^{\prime}\right) c^{-j-k-1} \\
& =M(c) B_{j}^{+}\left(y, \xi^{\prime}\right) c^{-j}
\end{aligned}
$$

and, similarly, $B_{j}^{-}\left(y, c \xi^{\prime}\right)=M(c) B_{j}^{-}\left(y, \xi^{\prime}\right) c^{-j}$. Hence (9) holds for $c>0$. In view of $\Delta_{-}\left(y, \xi^{\prime}\right)=M(-1) \Delta_{+}\left(y, \xi^{\prime}\right)$ and the equations (8), a similar calculation yields

$$
B_{j}^{+}\left(y,-\xi^{\prime}\right)=M(-1) B_{j}^{-}\left(y, \xi^{\prime}\right)(-1)^{-j}
$$


and

$$
B_{j}^{-}\left(y,-\xi^{\prime}\right)=M(-1) B_{j}^{+}\left(y, \xi^{\prime}\right)(-1)^{-j},
$$

so that (9) holds for $c=-1$, and thus for all $c \neq 0$. Finally, let $\mathcal{B}_{j}$ be an $r \times p$ matrix of classical p.d.o.'s on $\partial \Omega$ with principal symbol equal to $B_{j}$ (Theorem 1.4(ii)), and then let

$$
\mathcal{B}=\sum_{j=0}^{\ell-1} \mathcal{B}_{j}\left(y, D^{\prime}\right) D_{n}^{j}
$$

By construction the $k$ th row of $\mathcal{B}$ has order $m_{k}(k=1, \ldots, r)$ and $\Delta_{\mathcal{B}}^{ \pm}=\Delta_{ \pm}$. This completes the proof of the theorem, for the uniqueness follows from Prop. 5.2.

Remark 5.4. The $k$ th row of $\tilde{B}_{j}\left(y, \xi^{\prime}\right)$ has order $m_{k}-j$, where $j=0, \ldots, \ell-1$. If $m_{k} \geq \ell-1$ for all $k$, then all these entries have nonnegative order.

For elliptic operators in the plane $(n=2)$ on a simply connected domain $\Omega$, the "topological obstruction" mentioned above is proper ellipticity; that is, $\mathcal{A}$ has a boundary operator satisfying the L-condition if and only if it is properly elliptic. Necessity has been proved in the remarks following Def. 1.6, and sufficiency is proved in [Ro2, Theorem 2.4].

It should be noted that triviality of $\mathfrak{M}^{+}$is the basic restriction for existence of boundary operators only because we have assumed that the image of the boundary operator lies in the sections of a trivial bundle. If the boundary operators had been permitted in the form

$$
\mathcal{B}: C^{\infty}\left(\bar{\Omega}, \mathbb{C}^{p}\right) \longrightarrow \bigoplus_{j=1}^{J} C^{\infty}\left(\partial \Omega, G_{j}\right),
$$

where $G_{j}$ are vector bundles over $\partial \Omega$ (and the $j$ th component of $\mathcal{B}$ is of order $\left.m_{j}, j=1, \ldots, J\right)$, then a weaker restriction on $\mathfrak{M}^{+}$would have been obtained which is fundamental:

The necessary and sufficient condition for existence of a boundary operator $\mathcal{B}$ is that $\mathfrak{M}^{+} \simeq p_{*}^{-1} V$ for some vector bundle $V$ over $\partial \Omega$.

Here $p_{*}: \mathrm{ST}^{*}(\partial \Omega) \rightarrow \partial \Omega$ is the projection $\left(y, \xi^{\prime}\right) \mapsto y$ from the unit cotangent bundle to the boundary, and the condition on $\mathfrak{M}^{+}$means that the fibers $\mathfrak{M}_{y, \xi^{\prime}}^{+}=V_{y}$ are independent of $\xi^{\prime}$. One could, of course, allow the elliptic operator $\mathcal{A}$ to act in vector bundles too, i.e. $\mathcal{A}: C^{\infty}(\bar{\Omega}, E) \rightarrow C^{\infty}(\bar{\Omega}, F)$, where $E, F$ are vector bundles over $\bar{\Omega}$ with the same fiber dimension. We have chosen to fix attention on systems so that the connection with the matrix theory of $\S 2$ is more readily apparent.

The following is another version of Theorem 5.3. Note that the condition $G \cdot P_{+}^{\prime}=$ $G$ can always be achieved since we can replace $G$ by $G \cdot P_{+}^{\prime}$ (where $P_{+}^{\prime}=P_{\gamma^{+}}^{\prime}$ is the left Calderón projector).

Theorem 5.5. Let $G$ be a smooth $r \times p \ell$ matrix function on $\mathrm{ST}^{*}(\partial \Omega)$ such that at each point $\left(y, \xi^{\prime}\right) \in \mathrm{ST}^{*}(\partial \Omega)$ we have $G \cdot P_{+}^{\prime}=G$ and $G: \operatorname{im} P_{+}^{\prime} \rightarrow \mathbb{C}^{r}$ is invertible. Then for any $m_{k} \in \mathbb{R}, k=1, \ldots, r$, there exists a boundary operator $\mathcal{B}$ with transversal order $\mu \leq \ell-1$ such that the $k$ th row of $\pi \mathcal{B}$ is positive and negative homogeneous of degree $m_{k}$ and

$$
G=\frac{1}{2 \pi i} \int_{+} B(\lambda) L^{-1}(\lambda)\left[\begin{array}{lll}
I & \ldots & \lambda^{\ell-1} I
\end{array}\right] d \lambda .
$$


The principal part of $\mathcal{B}$ is uniquely determined by these conditions. Since the rank of $G$ is $r$, then $\mathcal{B}$ satisfies the L-condition.

Proof. First note that $\mathfrak{M}^{+}$is a trivial bundle, due to the isomorphism $G: \operatorname{im} P_{+}^{\prime} \rightarrow$ $\mathbb{C}^{r}$ and the natural isomorphism $\mathfrak{M}^{+} \simeq \mathrm{imP}_{+}^{\prime}$ (see Corollary 4.3). Therefore we can apply Theorem 5.3. Let $\left(X_{+}, T_{+}, Y_{+}\right)$be a $\gamma^{+}$-spectral triple for $L(\lambda)$ consisting of smooth matrix functions on $\mathrm{T}^{*}(\partial \Omega) \backslash 0$, with the usual homogeneity properties. Now, let $\Delta=G \cdot \mathcal{Z} \cdot \operatorname{col}\left(X_{+} T_{+}^{j}\right)_{j=0}^{\ell-1}$, which is an $r \times r$ matrix function on $\mathrm{ST}^{*}(\partial \Omega)$. In view of Corollary 2.12, we see that

$$
\Delta \cdot\left[\begin{array}{lll}
Y_{+} & \ldots & T_{+}^{\ell-1} Y_{+}
\end{array}\right]=G .
$$

Since $G$ is surjective, it follows that $\Delta$ is surjective at each point of $\mathrm{ST}^{*}(\partial \Omega)$. However, $\Delta$ is a square matrix, so $\operatorname{det} \Delta \neq 0$, and $\Delta$ defines a smooth matrix function $\mathrm{ST}^{*}(\partial \Omega) \rightarrow \mathrm{GL}_{r}(\mathbb{C})$. By Theorem 5.3 there exists a boundary operator $\mathcal{B}$ with $\Delta_{\mathcal{B}}^{+}=\Delta$. The equation (14) follows from (15). The boundary operator $\mathcal{B}$ is uniquely determined in view of Theorem 5.3.

We now return to discuss operators of general Douglis-Nirenberg type. Note that $\pi \mathcal{A}(x, c \xi)=S(c) \pi A(x, \xi) T(c)$ holds, where

$$
S(c)=\left[\delta_{i k} c^{s_{k}}\right], \quad T(c)=\left[\delta_{i k} c^{t_{k}}\right]
$$

are $p \times p$ diagonal matrices. It follows that

$$
L_{y, c \xi^{\prime}}(\lambda)=S(c) \cdot L_{y, \xi^{\prime}}\left(c^{-1} \lambda\right) \cdot T(c) ;
$$

hence the coefficients, $A_{j}$, of $L(\lambda)$ are homogeneous of degree $s_{i}+t_{k}-j$ in the $(i, k)$ entry $(k$ and $i=1, \ldots, p)$, that is,

$$
A_{j}\left(y, c \xi^{\prime}\right)=S(c) A_{j}\left(y, \xi^{\prime}\right) T(c) c^{-j}, \quad j=0, \ldots, \ell
$$

(for any $c \in \mathbb{C}$ ). Once again we let $s$ be the map from $\mathrm{T}^{*}(\partial \Omega) \backslash 0$ to $\mathrm{ST}^{*}(\partial \Omega)$ defined by $s:\left(y, \xi^{\prime}\right) \mapsto\left(y, \xi^{\prime} /\left|\xi^{\prime}\right|\right)$.

Lemma 5.6. There exists a $\gamma^{+}$-spectral triple $\left(X_{+}, T_{+}, Y_{+}\right)$for $L(\lambda)$ with the following properties for any $c>0$

$$
\begin{aligned}
X_{+}\left(y, c \xi^{\prime}\right) & =T^{-1}(c) X_{+}\left(y, \xi^{\prime}\right), \\
T_{+}\left(y, c \xi^{\prime}\right) & =c \cdot T_{+}\left(y, \xi^{\prime}\right), \\
Y_{+}\left(y, c \xi^{\prime}\right) & =c \cdot Y_{+}\left(y, \xi^{\prime}\right) S^{-1}(c),
\end{aligned}
$$

where $X_{+} \in \mathcal{L}\left(s^{-1} E, \mathbb{C}^{p}\right), T_{+} \in \mathcal{L}\left(s^{-1} E\right), Y_{+} \in \mathcal{L}\left(\mathbb{C}^{p}, s^{-1} E\right)$ and $s^{-1} E$ is the pullback of $E$ to $\mathrm{T}^{*}(\partial \Omega) \backslash 0$ of some vector bundle $E$ over $\mathrm{ST}^{*}(\partial \Omega)$. (E is necessarily isomorphic to $\mathfrak{M}^{+}$.)

Proof. The proof is by the same method as in Lemma 5.1, where

$$
\begin{aligned}
X_{+}\left(y, \xi^{\prime}\right) & =T^{-1}\left(\left|\xi^{\prime}\right|\right) \cdot X_{+}\left(y, \xi^{\prime} /\left|\xi^{\prime}\right|\right), \\
T_{+}\left(y, \xi^{\prime}\right) & =\left|\xi^{\prime}\right| \cdot T_{+}\left(y, \xi^{\prime} /\left|\xi^{\prime}\right|\right), \\
Y_{+}\left(y, \xi^{\prime}\right) & =\left|\xi^{\prime}\right| \cdot Y_{+}\left(y, \xi^{\prime} /\left|\xi^{\prime}\right|\right) \cdot S^{-1}\left(\left|\xi^{\prime}\right|\right) .
\end{aligned}
$$

Remark. The equations (3)-(7) still hold provided we define

$$
F(c)=\operatorname{diag}\left(c^{j} T^{-1}(c)\right)_{j=0}^{\ell-1}, \quad E(c)=c \cdot \operatorname{diag}\left(c^{j} S^{-1}(c)\right)_{j=0}^{\ell-1} .
$$


From now on it is always assumed that the $\gamma^{+}$-spectral triple of $L(\lambda)$ is chosen with the properties (17). By the same method as in the proof of Lemma 5.1 it is clear that the triple

$$
\begin{aligned}
& \left(X_{-}\left(y, \xi^{\prime}\right), T_{-}\left(y, \xi^{\prime}\right), Y_{-}\left(y, \xi^{\prime}\right)\right) \\
& \quad:=\left(T(-1) \cdot X_{+}\left(y,-\xi^{\prime}\right),-T_{+}\left(y,-\xi^{\prime}\right),-Y_{+}\left(y,-\xi^{\prime}\right) \cdot S(-1)\right)
\end{aligned}
$$

is a $\gamma^{-}$-spectral triple of $L_{y, \xi^{\prime}}(\lambda)$; it also has the homogeneity properties (17).

Let $\mathcal{B}$ be a general boundary operator. The $(k, i)$ entry of $\pi \mathcal{B}$ is homogeneous of degree $m_{k}+t_{i}$, so the $(k, i)$ entry of the coefficient $B_{j}$ of the corresponding matrix polynomial $B(\lambda)$ is homogeneous of degree $m_{k}+t_{i}-j$. In other words,

$$
B_{j}\left(y, c \xi^{\prime}\right)=M(c) B_{j}\left(y, \xi^{\prime}\right) T(c) c^{-j}, \quad c>0,
$$

for $j=0, \ldots, \mu$. We define $\Delta_{\mathcal{B}}^{+}$just as in (10), and then (11) holds without change. If $\pi \mathcal{B}$ is positive and negative homogeneous we define $\Delta_{\mathcal{B}}^{-}$as in $\left(10^{\prime}\right)$, and then $\left(11^{\prime}\right)$ holds.

In general for DN operators we have $2 r=\alpha<p \ell$, in which case the leading coefficient of $L(\lambda)$ is not invertible. We have a finite spectral pair $(X, T)$

$$
X=\left[\begin{array}{ll}
X_{+} & X_{-}
\end{array}\right], \quad T=\left(\begin{array}{cc}
T_{+} & \\
& T_{-}
\end{array}\right) \quad \text { and } \quad Y=\left(\begin{array}{c}
Y_{+} \\
Y_{-}
\end{array}\right),
$$

but $\operatorname{col}\left(X T^{j}\right)_{j=0}^{\ell-1}$ is not invertible. A pair of equations of the form $\sum_{j=0}^{\ell-1} B_{j} X_{ \pm} T_{ \pm}^{j}=$ $\Delta_{ \pm}$still has a solution for $B_{j}$ since $\mathcal{Z} \cdot \operatorname{row}\left(T^{j} Y\right)_{j=0}^{\ell-1}$ is a left inverse of $\operatorname{col}\left(X T^{j}\right)_{j=0}^{\ell-1}$, but the solution is not unique. Hence Theorems 5.3 and 5.5 still hold, except for the uniqueness (and of course the condition on the homogeneity of $\pi \mathcal{B}$ is modified: $\pi \mathcal{B}$ has DN numbers $m_{k}+t_{j}$ ).

\section{Two theorems of Agranovič and Dynin type}

Let $\mathrm{Ell}^{\mathrm{s}, \mathrm{t}}$ denote the set of $p \times p$ properly elliptic differential operators on $\bar{\Omega}$ with the DN numbers $s_{i}+t_{j}$, and let

$$
\mathrm{BE}^{\mathbf{s}, \mathbf{t}, \mathbf{m}}
$$

be the set of pairs $(\mathcal{A}, \mathcal{B})$, where $\mathcal{A} \in \mathrm{Ell}^{\mathbf{s}, \mathbf{t}}$ and $\mathcal{B}$ is a boundary operator on $\partial \Omega$ with DN numbers $m_{k}+t_{j}$ and satisfying the L-condition.

Notation. If we have two boundary value problems $\left(\mathcal{A}, \mathcal{B}^{i}\right) \in \mathrm{BE}^{\mathbf{s , t}, \mathbf{m}}, i=0,1$, with the same elliptic operator $\mathcal{A}$ such that $\Delta_{\mathcal{B}^{0}}^{+}=\Delta_{\mathcal{B}^{1}}^{+}$, we write

$$
\left(\mathcal{A}, \mathcal{B}^{0}\right) \approx\left(\mathcal{A}, \mathcal{B}^{1}\right)
$$

As stated in Theorem 1.7, an elliptic boundary problem defines a Fredholm operator between various Sobolev spaces. In the following lemma, we use the fact that the index of Fredholm operators is locally constant (with respect to the operator norm).

Lemma 6.1. If $\left(\mathcal{A}, \mathcal{B}^{0}\right) \approx\left(\mathcal{A}, \mathcal{B}^{1}\right)$ then

$$
\operatorname{ind}\left(\mathcal{A}, \mathcal{B}^{0}\right)=\operatorname{ind}\left(\mathcal{A}, \mathcal{B}^{1}\right)
$$

Proof. Let $\Delta_{\mathcal{B}^{i}}^{+}=\Delta$ for $i=0,1$. Consider the boundary operators $\mathcal{B}^{\tau}=\tau \mathcal{B}^{0}+$ $(1-\tau) \mathcal{B}^{1}, 0 \leq \tau \leq 1$. Since

$$
\Delta_{\tau \mathcal{B}^{0}+(1-\tau) \mathcal{B}^{1}}^{+}=\tau \cdot \Delta_{\mathcal{B}^{0}}^{+}+(1-\tau) \cdot \Delta_{\mathcal{B}^{1}}^{+}=\Delta
$$


is invertible, then by Theorem 3.3(iii) the boundary operators $\mathcal{B}^{\tau}$ satisfy the Lcondition relative to $\mathcal{A}$ for all $0 \leq \tau \leq 1$. Hence we have the linear homotopy

$$
\left(\mathcal{A}, \mathcal{B}^{\tau}\right)=\tau \cdot\left(\mathcal{A}, \mathcal{B}^{0}\right)+(1-\tau) \cdot\left(\mathcal{A}, \mathcal{B}^{1}\right)
$$

in the space of Fredholm operators $\mathcal{W}^{\ell+\mathbf{t}}(\Omega) \rightarrow \mathcal{W}^{\ell-\mathbf{s}}(\Omega) \times \mathcal{W}^{\ell-\mathbf{m}-1 / 2}(\partial \Omega)$, and the lemma follows immediately.

Sometimes it is convenient to modify the DN numbers of a boundary operator. Let $\Lambda^{m} \in \operatorname{OS}^{m}(\partial \Omega), m \in \mathbb{R}$, be a classical p.d.o. on $\partial \Omega$ with principal symbol $\left|\xi^{\prime}\right|^{m}$; then ind $\Lambda^{m}=0$. (Note that the index of $\Lambda^{m}$ is equal to that of its adjoint - and is hence zero-because the symbol of $\Lambda^{m}$ is real-valued, so it differs from its adjoint by an operator of lower order, $m-1$.) For $\mathbf{m} \in \mathbb{R}^{r}$ let

$$
\Lambda^{\mathbf{m}}=\left[\Lambda^{m_{k}} \delta_{k j}\right]_{k, j=1}^{r},
$$

which is a diagonal matrix operator, and ind $\Lambda^{\mathrm{m}}=0$ also. Given any boundary operator $\mathcal{B}=\sum_{j=0}^{\mu} \mathcal{B}_{j}\left(y, D^{\prime}\right) D_{n}^{j}$ with $\mathrm{DN}$ numbers $m_{k}+t_{j}$, the operator

$$
\mathcal{B}^{\prime}=\Lambda^{\tilde{\mathbf{m}}-\mathbf{m}} \circ \mathcal{B}=\sum_{j=0}^{\mu} \Lambda^{\tilde{\mathbf{m}}-\mathbf{m}} \mathcal{B}_{j}\left(y, D^{\prime}\right) D_{n}^{j}
$$

has DN numbers $\tilde{m}_{k}+t_{j}$, where $\tilde{\mathbf{m}} \in \mathbb{R}^{r}$ is chosen arbitrarily. (In particular we may let $\tilde{m}_{k}=0, k=1, \ldots, r$.) Clearly, $\mathcal{B}^{\prime}$ satisfies the L-condition relative to $\mathcal{A}$ if and only if $\mathcal{B}$ does. Since the index of a composition of Fredholm operators equals the sum of the indices of the individual operators, we have $\operatorname{ind}\left(\mathcal{A}, \mathcal{B}^{\prime}\right)=$ $\operatorname{ind}(I, \Lambda)+\operatorname{ind}(\mathcal{A}, \mathcal{B})$, whence

$$
\operatorname{ind}\left(\mathcal{A}, \mathcal{B}^{\prime}\right)=\operatorname{ind}(\mathcal{A}, \mathcal{B})
$$

The following theorem is due to Agranovič and Dynin (see $[\mathrm{Ag}]$ ).

Theorem 6.2. Let $\left(\mathcal{A}, \mathcal{B}_{i}\right), i=1,2$, be two boundary value problems $\in \mathrm{BE}^{\mathbf{s , t}, \mathbf{m}}$ having the same elliptic operator. Then

$$
\operatorname{ind}\left(\mathcal{A}, \mathcal{B}_{2}\right)=\operatorname{ind}\left(\mathcal{A}, \mathcal{B}_{1}\right)+\operatorname{ind} S
$$

where $S \in \operatorname{OS}^{0}(\partial \Omega, r \times r)$ with principal symbol $\pi S=\Delta_{\mathcal{B}_{2}}^{+} \cdot\left(\Delta_{\mathcal{B}_{1}}^{+}\right)^{-1}$ on $\operatorname{ST}^{*}(\partial \Omega)$.

Note. $\Delta_{\mathcal{B}_{1}}^{+}$and $\Delta_{\mathcal{B}_{2}}^{+}$are defined by $(5-10)$ with respect to any $\gamma^{+}$-spectral pair of $L(\lambda)=\pi \mathcal{A}\left(y,\left(\xi^{\prime}, \lambda\right)\right)$.

Proof. Observe that the $r \times r$ matrix function $\Delta_{\mathcal{B}_{2}}^{+} \cdot\left(\Delta_{\mathcal{B}_{1}}^{+}\right)^{-1}$ is independent of the choice of spectral pair, since any two such pairs are similar. In view of (1) we may assume that $\mathcal{B}_{1}$ and $\mathcal{B}_{2}$ have the same DN numbers, say $m_{k}=0$. By Theorem 1.4(ii) there exists a classical pseudo-differential operator $S \in \operatorname{OS}^{0}(\partial \Omega, r \times r)$ such that $\pi S=\Delta_{\mathcal{B}_{2}}^{+} \cdot\left(\Delta_{\mathcal{B}_{1}}^{+}\right)^{-1}$. Consider the operator $\left(\mathcal{A}, S \mathcal{B}_{1}\right)$; since $\Delta_{S \mathcal{B}_{1}}^{+}=\pi S \cdot \Delta_{\mathcal{B}_{1}}^{+}=\Delta_{\mathcal{B}_{2}}^{+}$, then by Lemma 6.1 we have

$$
\operatorname{ind}\left(\mathcal{A}, \mathcal{B}_{2}\right)=\operatorname{ind}\left(\mathcal{A}, S \mathcal{B}_{1}\right) .
$$

Now, since $\left(\mathcal{A}, S \mathcal{B}_{1}\right)=(I, S) \oplus\left(\mathcal{A}, \mathcal{B}_{1}\right)$, it follows that

$$
\operatorname{ind}\left(\mathcal{A}, S \mathcal{B}_{1}\right)=\operatorname{ind}(I, S)+\operatorname{ind}\left(\mathcal{A}, \mathcal{B}_{1}\right)=\operatorname{ind} S+\operatorname{ind}\left(\mathcal{A}, \mathcal{B}_{1}\right),
$$

so the proof is complete. 
The particular values of the matrix function $\Delta_{\mathcal{B}}^{+}$are of no significance because they depend on the choice of $\left(X_{+}, T_{+}\right)$. In fact, replacing $\left(X_{+}, T_{+}\right)$by the similar pair $\left(X_{+}\left(\Delta_{\mathcal{B}}^{+}\right)^{-1}, \Delta_{\mathcal{B}}^{+} T_{+}\left(\Delta_{\mathcal{B}}^{+}\right)^{-1}\right)$, we may assume that $\Delta_{\mathcal{B}}^{+}=I_{r}$, the $r \times r$ identity matrix. On the other hand, given two boundary operators $\mathcal{B}_{1}$ and $\mathcal{B}_{2}$ for the same elliptic operator $\mathcal{A}$, then the matrix $\Delta_{\mathcal{B}_{2}}^{+}\left(\Delta_{\mathcal{B}_{1}}^{+}\right)^{-1}$ does have a topological significance, as Theorem 6.2 indicates.

It should be mentioned that for an elliptic operator $\mathcal{A}$ which is defined on a simply connected region, $\bar{\Omega}$, in the plane $(n=2)$, there is an essentially canonical choice of $\left(X_{+}, T_{+}\right)$that depends on the values of the coefficients of $\mathcal{A}$ on all of $\bar{\Omega}$ (not just $\partial \Omega$ ). In this case $\Delta_{\mathcal{B}}^{+}$becomes an interesting function to study in its own right, and in fact the index of $(\mathcal{A}, \mathcal{B})$ can be expressed in terms of a winding number of $\Delta_{\mathcal{B}}^{+}$along $\operatorname{ST}^{*}(\partial \Omega)$. (See $\left.[\operatorname{Ro} 2].\right)$

We turn now to prove a result which in view of Lemma 6.1 implies that, for the purpose of index calculations, there is no loss of generality in considering only those boundary operators where the transversal order, $\mu$, is less than the degree, $\ell$, of the matrix polynomial $L(\lambda)=\pi \mathcal{A}\left(y,\left(\xi^{\prime}, \lambda\right)\right)$.

Theorem 6.3. Let $(\mathcal{A}, \mathcal{B}) \in \mathrm{BE}^{\mathbf{s , t}, \mathbf{m}}$. Then for some boundary operator $\mathcal{R}$ with transversal order $\mu \leq \ell-1$ and having the same $D N$ numbers as $\mathcal{B}$ we have

$$
(\mathcal{A}, \mathcal{B}) \approx(\mathcal{A}, \mathcal{R})
$$

i.e. $\mathcal{R}$ can be chosen so that $\Delta_{\mathcal{R}}^{+}=\Delta_{\mathcal{B}}^{+}$. Further, if $\pi \mathcal{B}$ is both positive and negative homogeneous, then $\mathcal{R}$ can be chosen so that $\Delta_{\mathcal{R}}^{ \pm}=\Delta_{\mathcal{B}}^{ \pm}$.

Proof. Let $\left(X_{+}, T_{+}, Y_{+}\right)$be a matrix $\gamma^{+}$-spectral triple of $L(\lambda)$ satisfying, as usual, the homogeneity properties (5-17), and $\left(X_{-}, T_{-}, Y_{-}\right)$the $\gamma^{-}$-spectral triple defined by (5-18). Suppose first that $\pi \mathcal{B}$ is both positive and negative homogeneous. We wish to choose matrix functions $R_{j}$ such that

$$
\sum_{j=0}^{\ell-1} R_{j}\left(y, \xi^{\prime}\right) X_{ \pm} T_{ \pm}^{j}=\sum_{j=0}^{\mu} B_{j}\left(y, \xi^{\prime}\right) X_{ \pm} T_{ \pm}^{j}
$$

Thus we let $R_{j}$ be defined by the expression on the right-hand side of (5-12), $j=0, \ldots, \ell-1$, or in other words

$$
\left[\begin{array}{lll}
R_{0} & \ldots & R_{\ell-1}
\end{array}\right]=\Delta_{\mathcal{B}}^{+} \cdot\left[\begin{array}{llll}
Y_{+} & \ldots & T_{+}^{\ell-1} Y_{+}
\end{array}\right] \mathcal{Z}+\Delta_{\mathcal{B}}^{-} \cdot\left[\begin{array}{llll}
Y_{-} & \ldots & T_{-}^{\ell-1} Y_{-}
\end{array}\right] \mathcal{Z} .
$$

It is clear that $R_{j}\left(y, c \xi^{\prime}\right)=M(c) R_{j}\left(y, \xi^{\prime}\right) T(c) c^{-j}$ for all $c \neq 0$ (see the proof of Theorem 5.3), so we let

$$
\mathcal{R}=\sum_{j=0}^{\ell-1} \mathcal{R}_{j}\left(y, D^{\prime}\right) D_{n}^{j}
$$

where $\mathcal{R}_{j} \in \mathrm{OS}^{\mathbf{m}+\mathbf{t}-j}(\partial \Omega, r \times p)$ is a pseudo-differential operator on $\partial \Omega$ with principal symbol $R_{j}, j=0, \ldots, \ell-1$. Then $\mathcal{R}$ has the same DN numbers as $\mathcal{B}$ and satisfies (2) by construction, since

$$
\Delta_{\mathcal{R}}^{+}=\left[\begin{array}{lll}
R_{0} & \cdots & R_{\ell-1}
\end{array}\right] \cdot \operatorname{col}\left(X_{+} T_{+}^{j}\right)_{j=0}^{\ell-1}=\Delta_{\mathcal{B}}^{+} \cdot I+\Delta_{\mathcal{B}}^{-} \cdot 0=\Delta_{\mathcal{B}}^{+},
$$

using Corollary 2.12 and the equation (2-12b). Similarly, $\Delta_{\mathcal{R}}^{-}=\Delta_{\mathcal{B}}^{-}$. In the case where $\pi \mathcal{B}$ is just positive homogeneous we define $R_{j}$ by the + terms on the 
right-hand side of (5-12), or, in other words,

$$
\left[\begin{array}{lll}
R_{0} & \ldots & R_{\ell-1}
\end{array}\right]=\Delta_{\mathcal{B}}^{+} \cdot\left[\begin{array}{lll}
Y_{+} & \ldots & T_{+}^{\ell-1} Y_{+}
\end{array}\right] \mathcal{Z},
$$

and then continue as before. The proof is complete.

Let $B(\lambda)=\pi \mathcal{B}\left(y,\left(\xi^{\prime}, \lambda\right)\right)$ and $R(\lambda)=\pi \mathcal{R}\left(y,\left(\xi^{\prime}, \lambda\right)\right)$ be the matrix polynomials associated with $\mathcal{B}$ and $\mathcal{R}$, respectively. If (2) holds, it follows that

$$
B(\lambda)=Q(\lambda) L(\lambda)+R(\lambda)
$$

for some matrix polynomial $Q(\lambda)$. Indeed, $(B(\lambda)-R(\lambda)) L^{-1}(\lambda)$ has an analytic continuation inside $\gamma^{+}$:

$$
\begin{aligned}
\frac{1}{2 \pi i} \int_{\gamma^{+}} \lambda^{j}(B(\lambda)-R(\lambda)) L^{-1}(\lambda) d \lambda= & {\left[\begin{array}{lll}
B_{0} & \ldots & B_{\mu}
\end{array}\right] \operatorname{col}\left(X_{+} T_{+}^{j}\right)_{j=0}^{\mu} \cdot T_{+}^{j} Y_{+} } \\
& \quad-\left[\begin{array}{lll}
R_{0} & \ldots & R_{\ell-1}
\end{array}\right] \operatorname{col}\left(X_{+} T_{+}^{k}\right)_{j=0}^{\ell-1} \cdot T_{+}^{j} Y_{+} \\
= & \Delta_{\mathcal{B}}^{+} \cdot T_{+}^{j} Y_{+}-\Delta_{\mathcal{R}}^{+} \cdot T_{+}^{j} Y_{+} \\
= & 0,
\end{aligned}
$$

for all $j=0,1, \ldots$, since $\Delta_{\mathcal{R}}^{+}=\Delta_{\mathcal{B}}^{+}$. In the same way, one also shows that it has an analytic continuation inside $\gamma^{-}$, so it has an analytic continuation to the finite complex plane. Since it has at most a pole at $\infty$, it follows that $(B(\lambda)-R(\lambda)) L^{-1}(\lambda)$ is a polynomial, or, in other words, (3) holds for some $r \times p$ matrix polynomial $Q(\lambda)$ with coefficients depending smoothly on the parameters $y, \xi^{\prime}$. Since $(\mathcal{A}, \mathcal{B}) \approx$ $(\mathcal{A}, \mathcal{R})$, then as in Lemma 6.1 we have the linear homotopy

$$
(\mathcal{A},(1-t) \mathcal{R}+t \mathcal{B}), \quad 0 \leq t \leq 1,
$$

joining $(\mathcal{A}, \mathcal{B})$ and $(\mathcal{A}, \mathcal{R})$ in the space $\mathrm{BE}^{\mathbf{s}, \mathbf{t}, \mathbf{m}}$. This homotopy corresponds to the following homotopy of matrix polynomials:

$$
\begin{aligned}
B^{t}(\lambda) & =(1-t) \cdot R(\lambda)+t \cdot B(\lambda) \\
& =t \cdot(B(\lambda)-R(\lambda))+R(\lambda) \\
& =t Q(\lambda) L(\lambda)+R(\lambda), \quad 0 \leq t \leq 1 .
\end{aligned}
$$

Remark. The equation (3) could be used for checking the L-condition. If (3) holds, then $(\mathcal{A}, \mathcal{B})$ satisfies the L-condition if and only if $(\mathcal{A}, \mathcal{R})$ satisfies the L-condition $\left(\right.$ since $\left.\Delta_{\mathcal{B}}^{+}=\Delta_{\mathcal{R}}^{+}\right)$.

\section{Homotopies OF ELLIPTIC BOUNDARY PROBLEMS}

Let $I$ be the unit interval $[0,1]$ in $\mathbb{R}$. A homotopy of elliptic differential operators is a family of $p \times p$ elliptic operators in Ell ${ }^{\mathbf{s}, \mathbf{t}}$ (i.e. with fixed DN numbers $s_{i}+t_{j}$ ),

$$
\mathcal{A}^{\tau}=\sum A_{\alpha}(\tau, x) D^{\alpha}, \quad \tau \in I,
$$

such that the coefficients $A_{\alpha}(t, \cdot)$ are continuous from $I$ to $C^{\infty}(\bar{\Omega}, p \times p)$.

If the coefficients $A_{\alpha}$ are $C^{\infty}$ functions of the variables $(\tau, x) \in I \times \bar{\Omega}$, then we say that the homotopy is a $C^{\infty}$ homotopy.

The following fact is important for the next lemma: We can "speed up" a homotopy $\mathcal{A}^{\tau}$ to make it constant in a neighbourhood of $\tau=1$ without affecting $\mathcal{A}^{0}$. 
Indeed, choose a function $\varphi \in C_{0}^{\infty}(\mathbb{R})$ such that $0 \leq \varphi \leq 1, \varphi(0)=0$ and $\varphi(\tau)=1$ when $1-\delta \leq \tau \leq 1$. Then for the homotopy $\overline{\mathcal{A}}^{\tau}=\mathcal{A}^{\varphi(\tau)}, 0 \leq \tau \leq 1$, we have

$$
\overline{\mathcal{A}}^{0}=\mathcal{A}^{0}, \quad \overline{\mathcal{A}}^{\tau}=\mathcal{A}^{1} \quad \text { when } 1-\delta \leq \tau \leq 1 .
$$

Similarly, we can "slow down" a homotopy to make it constant in a neighbourhood of $\tau=0$ without affecting its value at $\tau=1$.

Lemma 7.1. If two elliptic operators are homotopic, then they are $C^{\infty}$ homotopic.

Proof. Let $\mathcal{A}^{\tau}, 0 \leq \tau \leq 1$, be a (continuous) homotopy from $\mathcal{A}^{0}$ to $\mathcal{A}^{1}$. By definition, the maps $\tau \mapsto A_{\alpha}(\tau, \cdot)$ are continuous from $I$ to $C^{\infty}(\bar{\Omega}, p \times p)$, i.e. for any multi-index $\gamma$ we have

$$
\sup _{x \in \bar{\Omega}}\left\|D_{x}^{\gamma}\left[A_{\alpha}(\tau, x)-A_{\alpha}\left(\tau_{0}, x\right)\right]\right\|<\epsilon
$$

if $\left|\tau-\tau_{0}\right|$ is sufficiently small. With $\gamma=0$ this shows that $A_{\alpha}$ is continuous in $\tau$ uniformly with respect to $x$. Since $A_{\alpha}$ is continuous (in fact, smooth) in $x$ for fixed $\tau$, it follows that the matrix functions $A_{\alpha}$ are continuous on $I \times \bar{\Omega}$. For any $\epsilon>0$ we can find $\tilde{A}_{\alpha} \in C^{\infty}(I \times \bar{\Omega})$ such that

$$
\sup _{(\tau, x) \in I \times \bar{\Omega}}\left\|\tilde{A}_{\alpha}(\tau, x)-A_{\alpha}(\tau, x)\right\|<\epsilon .
$$

Then $\tilde{\mathcal{A}}^{\tau}:=\sum \tilde{A}_{\alpha}(\tau, x) D^{\alpha}, 0 \leq \tau \leq 1$, is a $C^{\infty}$ homotopy of elliptic operators (ellipticity is preserved if $\epsilon$ is chosen sufficiently small), and, further, we can join $\tilde{\mathcal{A}}^{i}$ and $\mathcal{A}^{i}, i=0,1$, by a linear homotopy

$$
\kappa \tilde{\mathcal{A}}^{i}+(1-\kappa) \mathcal{A}^{i}, \quad 0 \leq \kappa \leq 1 .
$$

Finally, we can join the three homotopies to get

$$
\begin{cases}\kappa \tilde{\mathcal{A}}^{0}+(1-\kappa) \mathcal{A}^{0}, & 0 \leq \kappa \leq 1, \\ \tilde{\mathcal{A}}^{\kappa-1}, & 1 \leq \kappa \leq 2, \\ (3-\kappa) \tilde{\mathcal{A}}^{1}+(\kappa-2) \mathcal{A}^{1}, & 2 \leq \kappa \leq 3,\end{cases}
$$

which is a piecewise $C^{\infty}$ homotopy from $\mathcal{A}^{0}$ to $\mathcal{A}^{1}$. It is piecewise $C^{\infty}$ only because of the points $\kappa=1$ and $\kappa=2$, but it becomes $C^{\infty}$ if we first modify the homotopies to make them constant in a neighbourhood of these points, as explained above.

Lemma 7.2. Let $\mathcal{A}^{\tau}, \tau \in I$, be a homotopy of elliptic operators and suppose that $\mathcal{A}^{0}$ has a boundary operator satisfying the L-condition. Then there exists a matrix $\gamma^{+}$-spectral triple

$$
\left(X_{+}\left(\tau, y, \xi^{\prime}\right), T_{+}\left(\tau, y, \xi^{\prime}\right), Y_{+}\left(\tau, y, \xi^{\prime}\right)\right)
$$

of $L_{\tau, y, \xi^{\prime}}(\lambda):=\pi \mathcal{A}^{\tau}\left(y,\left(\xi^{\prime}, \lambda\right)\right)$ satisfying the homogeneity properties (5-2), with entries that are continuous functions from I to $C^{\infty}\left(\mathrm{T}^{*}(\partial \Omega) \backslash 0\right)$. Further, if $\mathcal{A}^{\tau}$ is a $C^{\infty}$ homotopy, then there exists a matrix $\gamma^{+}$spectral triple with entries in $C^{\infty}\left(I \times \mathrm{T}^{*}(\partial \Omega) \backslash 0\right)$.

Proof. We will prove the lemma only for the case of $C^{\infty}$ homotopy. The general case follows in the same way once the results of $\S 4$ are generalized appropriately. Let $\mathfrak{M}^{+}$denote the vector bundle over $I \times \mathrm{ST}^{*}(\partial \Omega)$ with fibers $\mathfrak{M}_{L_{\tau, y, \xi^{\prime}}}^{+}$. Since the restriction of $\mathfrak{M}^{+}$to $\{0\} \times \mathrm{ST}^{*}(\partial \Omega)$ is trivial due to the existence of the boundary operator for $\mathcal{A}^{0}$ satisfying the L-condition, then $\mathfrak{M}^{+}$is also trivial (see [WRL, $\S 5.9]$ ). Hence there exists a $\gamma^{+}$-spectral triple (1) of $L_{\tau, y, \xi^{\prime}}(\lambda)$ consisting of matrix 
functions with entries that are $C^{\infty}$ functions on $I \times \operatorname{ST}^{*}(\partial \Omega)$. The operators $\mathcal{A}^{\tau}$ have the same DN numbers for all $\tau \in I$; hence as in Lemma 5.6 we can extend this triple by homogeneity (5-17) so that it is a $\gamma^{+}$-spectral triple of $L_{\tau, y, \xi^{\prime}}(\lambda)$ for all $\tau \in I$ and $\left(y, \xi^{\prime}\right) \in \mathrm{T}^{*}(\partial \Omega) \backslash 0$.

Remark. If $\mathcal{B}^{0}$ is a boundary operator for $\mathcal{A}^{0}$ satisfying the L-condition, then by replacing $\left(X_{+}, T_{+}, Y_{+}\right)$with $\left(X_{+} M^{-1}, M T_{+} M^{-1}, M Y_{+}\right)$, where $M=\Delta_{\mathcal{B}^{0}}^{+}$, we may assume that

$$
\Delta_{\mathcal{B}^{0}}^{+}=r \times r \text { identity matrix, }
$$

where $\Delta_{\mathcal{B}^{0}}^{+}$is given by $(5-10)$ with respect to the $\gamma^{+}$-spectral pair $\left(X_{+}(0, \cdot), T_{+}(0, \cdot)\right)$.

A homotopy of boundary problems is a family $\left(\mathcal{A}^{\tau}, \mathcal{B}^{\tau}\right)$ of boundary value problems in $\mathrm{BE}^{\mathbf{s}, \mathbf{t}, \mathbf{m}}$, i.e. satisfying the L-condition, where $\mathcal{A}^{\tau}$ is a homotopy in $\mathrm{El}^{\mathbf{s}, \mathbf{t}}$ and, in addition,

$$
\mathcal{B}^{\tau}=\sum \mathcal{B}_{j}^{(\tau)}\left(y, D^{\prime}\right) D_{n}^{j}, \quad \tau \in I
$$

where each $\mathcal{B}_{j}^{(\tau)} \in \mathrm{OS}^{\mathbf{m}+\mathbf{t}-j}(\partial \Omega, r \times p)$ is a classical p.d.o. with principal symbol $B_{j}(\tau, \cdot)$ whose restriction to the unit cotangent bundle $\mathrm{ST}^{*}(\partial \Omega)$ is continuous from $I$ to $C^{\infty}\left(\mathrm{ST}^{*}(\partial \Omega), r \times p\right)$. It is called a $C^{\infty}$ homotopy if $\mathcal{A}^{\tau}$ is a $C^{\infty}$ homotopy and the principal symbol of the operators $\mathcal{B}_{j}^{(\tau)}$ are $C^{\infty}$ functions of the variables $\left(\tau, y, \xi^{\prime}\right) \in I \times \mathrm{ST}^{*}(\partial \Omega)$. If two boundary value problems are homotopic then they are $C^{\infty}$ homotopic. (As in Lemma 7.1 a continuous homotopy of principal symbols implies existence of a $C^{\infty}$ homotopy, and then we may apply Theorem 1.4(ii) to lift the result to the operator level.)

The next theorem is the main result of this section. We have a map $\mathrm{BE}^{\mathbf{s , t}, \mathbf{m}} \rightarrow$ Ell ${ }^{\mathbf{s}, \mathbf{t}}$ defined by $(\mathcal{A}, \mathcal{B}) \mapsto \mathcal{A}$, and we show that a given homotopy $\mathcal{A}^{\tau}$ of elliptic operators in $\mathrm{Ell}^{\mathbf{s}, \mathbf{t}}$ can be lifted to a homotopy of boundary value problems in $\mathrm{BE}^{\mathbf{s}, \mathbf{t}, \mathbf{m}}$.

Theorem 7.3. Let $\mathcal{A}^{\tau}, 0 \leq \tau \leq 1$, be a homotopy (or $C^{\infty}$ homotopy) of $p \times p$ elliptic differential operators in $\mathrm{Ell}^{\mathbf{s}, \mathbf{t}}$, i.e. with the $D N$ numbers $s_{i}+t_{j}$. If $\mathcal{B}$ is a boundary operator satisfying the L-condition relative to $\mathcal{A}^{0}$ with DN numbers $m_{k}+t_{j}$, then there exists a homotopy (or $C^{\infty}$ homotopy) of boundary value problems $\left(\mathcal{A}^{\tau}, \mathcal{B}^{\tau}\right) \in \mathrm{BE}^{\mathbf{s}, \mathbf{t}, \mathbf{m}}, 0 \leq \tau \leq 1$, with $\left(\mathcal{A}^{0}, \mathcal{B}^{0}\right) \approx\left(\mathcal{A}^{0}, \mathcal{B}\right)$.

Remarks.

(i) In particular, it follows that there exists a boundary operator $\mathcal{B}^{1}$ satisfying the L-condition relative to $\mathcal{A}^{1}$.

(ii) The condition $\left(\mathcal{A}^{0}, \mathcal{B}^{0}\right) \approx\left(\mathcal{A}^{0}, \mathcal{B}\right)$ implies that the two boundary value problem operators are (linearly) homotopic, as in the proof of Lemma 6.1. Then by joining this homotopy with the homotopy $\left(\mathcal{A}^{\tau}, \mathcal{B}^{\tau}\right)$, as in the proof of Lemma 7.1 , we obtain a homotopy from the given boundary value problem operator, $\left(\mathcal{A}^{0}, \mathcal{B}\right)$, to the new one $\left(\mathcal{A}^{1}, \mathcal{B}^{1}\right)$.

Proof. We prove the theorem for $C^{\infty}$ homotopies since that is sufficient for our purpose (and, besides, if a homotopy is given, then a $C^{\infty}$ homotopy exists as shown above, so there is little loss of generality). By Lemma 7.2 there exists a $\gamma^{+}$-spectral triple (1) with the properties indicated there. Also, we may define a $\gamma^{-}$-spectral triple by the formula (5-18) and then a finite spectral triple $(X, T, Y)$ as in the proof of Theorem 5.3 (see (5-13)); we need this triple in order to use (2-14). The 
matrices $X, T$ and $Y$ are $C^{\infty}$ matrix functions on $I \times \mathrm{T}^{*}(\partial \Omega) \backslash 0$. As in Theorem 5.3 , there exist matrix functions $B_{j}$ such that

$$
\sum_{j=0}^{\ell-1} B_{j}(\tau, \cdot) X_{+}(\tau, \cdot) T_{+}^{j}(\tau, \cdot)=\Delta_{\mathcal{B}}^{+}
$$

for all $\tau \in I$, namely,

$$
B_{j}(\tau, \cdot)=\Delta_{\mathcal{B}}^{+} \cdot \sum_{k=0}^{\ell-j-1} T_{+}^{k}(\tau, \cdot) Y_{+}(\tau, \cdot) A_{j+k+1}(\tau, \cdot)
$$

(i.e. let $M_{+}=\Delta_{\mathcal{B}}^{+}$and $M_{-}=0$ in the formula $(2-14)$ ). Then

$$
B_{j} \in C^{\infty}\left(I \times \mathrm{T}^{*}(\partial \Omega) \backslash 0, r \times p\right),
$$

and, in view of the homogeneity properties (5-11), (5-16) and (5-17), it is easily seen that

$$
B_{j}\left(\tau, y, c \xi^{\prime}\right)=M(c) B_{j}\left(\tau, y, \xi^{\prime}\right) T(c) c^{-j}, \quad c>0,
$$

for all $j=0, \ldots, \ell-1$. Now let $\mathcal{B}_{j}^{(\tau)} \in \operatorname{OS}^{\mathbf{m}+\mathbf{t}-j}(\partial \Omega, r \times p)$ be a classical pseudodifferential operator on $\partial \Omega$ with principal symbol $B_{j}(\tau, \cdot)$. Then the boundary operator $\mathcal{B}^{\tau}=\sum_{j=0}^{\ell-1} \mathcal{B}_{j}^{(\tau)} D_{n}^{j}$ has the same DN numbers as $\mathcal{B}$; further, the pair $\left(\mathcal{A}^{\tau}, \mathcal{B}^{\tau}\right)$ satisfies the L-condition for all $\tau \in I$ due to (2) and Theorem 3.3(iii), so it is a homotopy in $\mathrm{BE}^{\mathbf{s , t}, \mathbf{m}}$. This completes the proof of the theorem, because in view of $(2)$ with $\tau=0$ we have $\Delta_{\mathcal{B}^{0}}^{+}=\Delta_{\mathcal{B}}^{+}$, i.e. $\left(\mathcal{A}, \mathcal{B}^{0}\right) \approx(\mathcal{A}, \mathcal{B})$.

Remark. Just as in Theorem 6.3, if $\pi \mathcal{B}^{0}$ is positive and negative homogeneous then the boundary operators $\mathcal{B}^{\tau}$ can also be constructed with this property. We let $M_{+}=\Delta_{\mathcal{B}}^{+}\left(y, \xi^{\prime}\right), M_{-}=\Delta_{\mathcal{B}}^{-}\left(y, \xi^{\prime}\right)$ in the formula (2-14) to obtain a solution of the equations

$$
\sum_{j=0}^{\ell-1} B_{j}(\tau, \cdot) X_{ \pm}(\tau, \cdot) T_{ \pm}^{j}(\tau, \cdot)=\Delta_{\mathcal{B}}^{ \pm}
$$

of the form

$$
\begin{aligned}
B_{j}(\tau, \cdot)=\Delta_{\mathcal{B}}^{+} & \cdot \sum_{k=0}^{\ell-j-1} T_{+}^{k}(\tau, \cdot) Y_{+}(\tau, \cdot) A_{j+k+1}(\tau, \cdot) \\
& +\Delta_{\mathcal{B}}^{-} \cdot \sum_{k=0}^{\ell-j-1} T_{-}^{k}(\tau, \cdot) Y_{-}(\tau, \cdot) A_{j+k+1}(\tau, \cdot),
\end{aligned}
$$

and then continue as before.

\section{REFERENCES}

[Ad] R.A. Adams, Sobolev Spaces, Academic Press, New York, 1975. MR 56:9247

[ADN] S. Agmon, A. Douglis, L. Nirenberg, Estimates near the boundary for solutions of elliptic partial differential equations satisfying general boundary conditions, Comm. Pure Appl. Math. 12 (1959), 623-727; II, Comm. Pure Appl. Math. 17 (1964), 35-92. MR 23:A2610; MR 28:5252

[Ag] M.S. Agranovič, Elliptic singular integro-differential operators, Russian Math. Surveys 20 (5) (1965), 1-122. MR 33:6176 
[BGR] J.A. Ball, I. Gohberg, L. Rodman, Interpolation of Rational Matrix Functions, Operator Theory: Advances and Applications 45, Birkhäuser Verlag, Basel and Boston, 1990. MR 92m: 47027

[Fe] B.V. Fedosov, An analytic formula for the index of an elliptic boundary-value problem, Math. USSR Sbornik 22 (1974), 61-90. MR 49:6285

[Ge] I. M. Gel'fand, On elliptic equations, Russian Math. Surveys 15 (3) (1960), 113-123. MR 23:A416

[GLR] I. Gohberg, P. Lancaster, L. Rodman, Matrix Polynomials, Academic Press, New York, 1982. MR 84c: 15012

[Ho] L. Hörmander, The Analysis of Linear Partial Differential Operators, vol. III, SpringerVerlag, New York and Berlin, 1985. MR 87d:35002a

[Lo] Ya. B. Lopatinskii, On a method of reducing boundary value problems for a system of differential equations of elliptic type to regular integral equations, Amer. Math. Soc. Transl. (Ser. 2) 89 (1970), 149-183. MR 17:494b

[LT] P. Lancaster, M. Tismenetsky, The Theory of Matrices with Applications, Academic Press, New York, 1985. MR 87a:15001

[R] L. Rodman, An Introduction to Operator Polynomials, OT 38, Birkhäuser Verlag, Basel and Boston, 1989. MR 90k:47032

[Ro1] B. Rowley, Wiener-Hopf factorization of operator polynomials, Integral Equations and Operator Theory 3 (3) (1980), 437-462. MR 81i:47023

[Ro2] _ An index formula for elliptic systems in the plane, Trans. Amer. Math. Soc. 349 (1997), 3149-3179.

[Se] R. Seeley, Singular integrals and boundary value problems, Amer. J. Math. 88 (1966), 781-809. MR 35:810

[Wl] J. Wloka, Partial Differential Equations, Cambridge University Press, 1987. MR 88d:35004

[WRL] J. Wloka, B. Rowley, B. Lawruk, Boundary Value Problems for Elliptic Systems, Cambridge University Press, 1995. MR 96f:35003

Department of Mathematics, Champlain College, Lennoxville, Quebec, Canada

E-mail address: browley@lennox.champlaincollege.qc.ca 